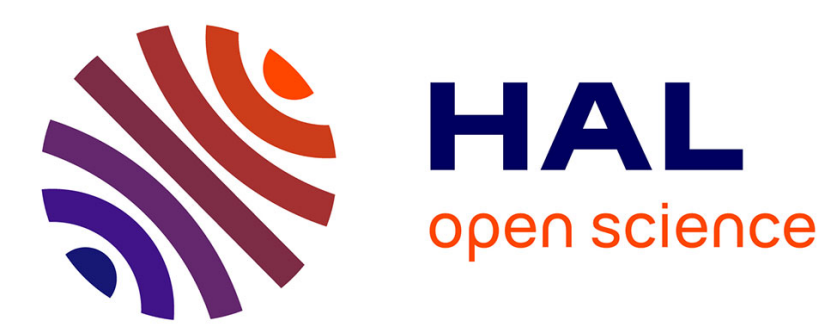

\title{
Numerical comparison between the Boltzmann and ES-BGK models for rarefied gases
}

Pierre Andries, Jean-François Bourgat, Patrick Le Tallec, Benoît Perthame

\section{To cite this version:}

Pierre Andries, Jean-François Bourgat, Patrick Le Tallec, Benoît Perthame. Numerical comparison between the Boltzmann and ES-BGK models for rarefied gases. [Research Report] RR-3872, INRIA. 2000. inria-00072782

\section{HAL Id: inria-00072782 \\ https://hal.inria.fr/inria-00072782}

Submitted on 24 May 2006

HAL is a multi-disciplinary open access archive for the deposit and dissemination of scientific research documents, whether they are published or not. The documents may come from teaching and research institutions in France or abroad, or from public or private research centers.
L'archive ouverte pluridisciplinaire HAL, est destinée au dépôt et à la diffusion de documents scientifiques de niveau recherche, publiés ou non, émanant des établissements d'enseignement et de recherche français ou étrangers, des laboratoires publics ou privés. 


\title{
Numerical comparison between the Boltzmann and ES-BGK models for rareøed gases
}

\author{
Pierre Andries - Jean-Franois Bourgat - Patrick Le Tallec - Benoit Perthame
}

N 3872

January 31,2000

THME 4 



\title{
Numerical comparison between the Boltzmann and ES-BGK models for rarefied gases
}

\author{
Pierre Andries* - Jean-François Bourgat ${ }^{\dagger}$ - Patrick Le Tallec ${ }^{\ddagger}$ - Benoit \\ Perthame $^{\S}$
}

Thème 4 - Simulation et optimisation

de systèmes complexes

Projet M3N

Rapport de recherche $n^{\circ} 3872$ - January 31,2000 - 48 pages

\begin{abstract}
Rarefied gas flows obey to the Boltzmann equation, but numerical simulations of this equation are not always possible, so that simpler models have been introduced. The ES-BGK equation is one of these models. It gives the correct transport coefficients for the Navier Stokes approximation, so that Boltzmann or ES-BGK simulations are expected to give the same results for dense gases, but in the case of a rarefied flow, complete numerical comparisons are needed.

In this paper we present numerical comparisons between the two models in transitional regimes (where the ES-BGK model is expected to be useful) for reentry flows around a compression ramp and a plate. We also emphasize that the ES-BGK model gives flow predictions closer to the Boltzmann result than the simpler BGK model.
\end{abstract}

Key-words: Kinetic theory, Monte Carlo simulation, Random particle models, BGK equations

This work has been supported by CEA-CESTA.

* M3N,email: pierre.andries@inria.fr

† M3N,email: jean-francois.bourgat@inria.fr

$\ddagger$ CEREMADE,Université Paris-Dauphine, 75775-Paris cedex 16, email: letallec@ceremade.dauphine.fr

$\S$ M3N,email: benoit.perthame@ens.fr 


\section{Comparaison entre les modèles de Boltzmann et de ES-BGK pour les gaz raréfiés}

Résumé : Les écoulements de gaz raréfiés obéissent à l'équation de Boltzmann, néanmoins, compte tenu de sa complexité, les simulations numériques de cette équation ne sont pas toujours possibles, et des modèles simplifiés ont donc été introduits. L'équation de ES-BGK fait partie de ces modèles simplifiés. Elle donne des coefficients de transport corrects dans l'approximation par les équations de Navier-Stokes quand le gaz est dense. Dans ce cas une simulation Boltzmann ou ES-BGK donne les mêmes résultats, mais des comparaisons numériques complètes sont nécessaires pour des gaz raréfiés.

Dans ce rapport, nous présentons des comparaisons numériques entre les deux modèles pour des régimes transitionnels (l'équation de ES-BGK semble alors la plus utile) dans le cas d'écoulements autour de corps de réentrée (rampe de compression, plaque). Nous insistons sur le fait que le modèle ES-BGK donne des résultats plus proches de ceux de Boltzmann qu'un autre modèle de relaxation, le modèle BGK.

Mots-clés : Théorie cinétique, Simulation de Monte Carlo, Méthodes particulaires, Equations de BGK 


\section{Contents}

1 Introduction 4

2 The models of rarefied flows $\quad 5$

2.1 The Boltzmann equation . . . . . . . . . . . . . . 5

2.2 The BGK model . . . . . . . . . . . . . . . 6

2.3 The ES-BGK model . . . . . . . . . . . . . . 7

2.4 The polyatomic ES-BGK model . . . . . . . . . . . 8

3 The numerical method $\quad \mathbf{1 0}$

3.1 The random particle method for solving Boltzmann equation . . . . 10

3.2 Random method for the ES-BGK model . . . . . . . . . . . . . 11

3.2.1 Collision probability of a cell . . . . . . . . . . . . . 12

3.2.2 Gaussian collision of the cell particles . . . . . . . . 13

4 Numerical comparison between ES-BGK and Boltzmann models 15

4.1 The plate test case . . . . . . . . . . . . . . 15

4.1.1 Plate data . . . . . . . . . . . . . . 15

4.1.2 Results of the plate test case for $K n=510^{-3} \ldots \ldots \ldots$

4.1.3 Plate results for $K n=1.4410^{-2} \ldots \ldots \ldots 17$

4.2 The ramp test case . . . . . . . . . . . . . . . . . . 17

4.2 .1 Ramp data . . . . . . . . . . . . . 17

4.2 .2 Results of the ramp test case . . . . . . . . . 18

$\begin{array}{lll}5 & \text { Study of the velocity distribution function } & 19\end{array}$

6 Conclusion $\quad 20$ 
P. Andries, J.F. Bourgat, P. Le Tallec, B. Perthame

\section{Introduction}

Rarefied gas flows obey the Boltzmann equation

$$
\partial_{t} f+v \cdot \nabla_{x} f=Q(f) .
$$

Because of the quadratic aspect of the kernel $Q(f)$ and the multiple integrations in its analytical formulation, this equation is complicated and difficult to compute. Henceforth different simpler models have been introduced, especially the BGK model [5] which is mainly a relaxation towards a maxwellian equilibrium state.

$$
\partial_{t} f+v \cdot \nabla_{x} f=\lambda(M[f]-f) .
$$

Although it describes the right hydrodynamical limit, the BGK model does not give the Navier Stokes equation with correct transport coefficients in the ChapmanEnskog expansion, and to correct this defect the so called ellipsoidal statistics (or ES-BGK) model was introduced by Holway [11]. It is a relaxation towards a Gaussian distribution (see section 2 for details)

$$
\partial_{t} f+v \cdot \nabla_{x} f=\lambda(G[f]-f) .
$$

Our new interest for this model is originated from the recent theoritical progress in [2] where the entropy inequality is proved.

The ES-BGK model appears now satisfying from a theoritical point of view, but since it is not derived from the Boltzmann equation it is not so sure in which extent it describes correctly rarefied gas flows. On the other hand the BGK model has been used numerically extensively, with very satisfactory results. For example in Aoki and al.[3] the flow around a plate is studied, precise numerical computation can be found in Loyalka [18], see also [9]. We can expect the ES-BGK model to approximate at least Boltzmann flows as well as the BGK model does, and to be able to cover a wider range of parameters. On the other hand, it was usually thought that the ES-BGK model does not satisfy the entropy inequality (H-theorem). Therefore no recent extensive numerical comparison between BGK, ES-BGK and Boltzmann models have been performed in the literature. Here we aim to show that, for a similar complexity, ES-BGK is more accurate than BGK model.

We compare some numerical simulations for a rarefied gas flow using the Boltzmann, BGK and ES-BGK models on two different test cases: flows around a plate 
and around a ramp. The simulation is based on a random particle method as explained in [6],[13]. A special emphasis of our numerical tests is a comparison for small knudsen number in the boundary layer.

Although the numerical method we use here is rather classical, we would like to emphasize that the validation of simpler models also aims to use recent methods which could be more efficient in transitional regimes, where the ES-BGK model seems to perform well enough. As examples of recent improvements at the numerical level, we would like to indicate an implicit method by Pareschi and al. [21], determinist schemes by Rogier and Schneider [23], Mieussens [19] (which actually motivated this work).

The outline of the paper is as follows: in section 2 we briefly recall the underlying models and equations which are studied numerically, in section 3 we present the outlines of the numerical scheme. The results of two different test cases (plate and ramp) are presented in section 4 . Section 5 is devoted to the study and comparison of the distribution functions numerically obtained.

\section{The models of rarefied flows}

For simplicity we first present the Boltzmann, BGK and ES-BGK equation for monoatomic gases. We also present briefly the polyatomic ES-BGK model, wich is completely described in [2]. The polyatomic Boltzmann model used in the computations classically follows the ideas of Borgnakke-Larsen ([15], see also [7]) and we do not present it here.

The equations for rarefied flows are of kinetic type, this means that the variables are the time $t \geq 0$, the position $x \in I R^{3}$ and the velocity $v \in I R^{3}$.

\subsection{The Boltzmann equation}

The Boltzmann equation can be written under the form (1.1) where Boltzmann's collision operator $Q_{B}(f)(x, v, t)$, which describes the evolution of the distribution function due to binary collisions between particles, can be written, using standard notations (see Cercigani [9], Lions [17])

$$
Q_{B}(f)(x, v, t)=\int_{v^{*} \in I R^{3}} \int_{\omega \in S^{2}}\left(f^{\prime} f^{\prime *}-f f^{*}\right) b\left(\left|v-v^{*}\right|, \omega\right) d v^{*} d \omega
$$

$\mathrm{RR} \mathrm{n}^{\circ} 3872$ 
It conserves mass, momentum and energy

$$
\int_{v \in R^{3}} Q_{B}(f)(x, v, t) \phi(v) d v=0, \text { for } \phi(v)=\left\{1, v, \frac{1}{2}|v|^{2}\right\},
$$

and satisfies the local entropy dissipation inequality

$$
\partial_{t} \int H(f) d v+\operatorname{div} \int v H(f) d v \leq 0,
$$

where $H($.$) denotes Boltzmann's entropy functional$

$$
H(f)=f \ln f .
$$

\subsection{The BGK model}

These last properties $(2.2,2.3)$ are also true for the simpler BGK operator which describes the relaxation of $f$ to the local Maxwellian equilibrium

$$
\begin{gathered}
\mathcal{M}[f]=\frac{\rho}{(2 \pi R T)^{3 / 2}} \exp \left(-\frac{|\mathrm{v}-\mathrm{u}|^{2}}{2 \mathrm{RT}}\right) . \\
Q_{B G K}(f)=\frac{P}{\mu}(\mathcal{M}[f]-f),
\end{gathered}
$$

It is defined by using the macroscopic density $\rho$, velocity $\underline{u}$ and translational temperature $T$ of the original non-negative distribution $f$ obtained through the moments

$$
\begin{gathered}
\rho(x, t)=<f>, \\
\underline{u}(x, t)=\frac{1}{\rho}<v f>, \\
R T=\frac{1}{3 \rho(x, t)}<|\underline{c}|^{2} f>,
\end{gathered}
$$

with the notations

$$
<f>(x, t)=\int_{R^{3}} f(x, t, v) d v, \quad \underline{c}=v-\underline{u} .
$$

Using this collision operator (2.5), a first order Chapman-Enskog expansion applied to the BGK equation gives the correct Navier-Stokes viscosity $\mu$. But, the associated heat conduction is given by $\kappa=\frac{5}{2} R \mu$ which leads to a Prandtl number given by

$$
\operatorname{Pr}=\frac{5}{2} \frac{R \mu}{\kappa}=1 \text {. }
$$


For most gases, we have $P r \leq 1$. In particular, for a monoatomic gas, the maxwellian model for Boltzmann collision operator leads to $\operatorname{Pr}=2 / 3$. Thus, this simple approximation will generally not give the correct Navier-Stokes heat conduction.

\subsection{The ES-BGK model}

In order to introduce the ES-BGK model, we need further notations. We define

$$
\begin{gathered}
P=\rho R T \quad \text { (kinetic pressure), } \\
E=\frac{1}{2}<|v|^{2} f>=\frac{1}{2} \rho\left(|u|^{2}+3 R T\right) \quad \text { (total energy), }
\end{gathered}
$$

and the opposite of the stress tensor

$$
\underline{\underline{\Theta}}=\frac{1}{\rho}<\underline{c} \otimes \underline{c f}>\text {. }
$$

Therefore the translational temperature is related to the stress tensor by the relation

$$
T=\frac{1}{3 R} \operatorname{tr} \underline{\underline{\Theta}} \text {. }
$$

We finally introduce the corrected tensor

$$
\underline{\underline{\mathcal{T}}}=(1-\nu) R T \underline{\underline{I d}}+\nu \underline{\underline{\Theta}},
$$

which can be viewed as a linear combination of the initial stress tensor $\underline{\underline{\Theta}}$ and of the isotropic stress tensor $R T \underline{\underline{I d}}$ developed by a Maxwellian distribution.

The ES-BGK model introduces a corrected BGK collision operator by replacing the local equilibrium Maxwellian by the Gaussian $\mathcal{G}[f]$ defined by

$$
\mathcal{G}[f]=\frac{\rho}{\sqrt{\operatorname{det}(2 \pi \underline{\mathcal{T}})}} \exp \left(-\frac{1}{2}(\underline{v}-\underline{u}) \cdot \underline{\underline{T}}^{-1} \cdot(\underline{v}-\underline{u})\right) .
$$

The corresponding collision operator is now

$$
Q_{E S}(f)=\frac{P}{\mu(1-\nu)}(\mathcal{G}[f]-f),
$$

where the parameter $-\frac{1}{2} \leq \nu<1$ is used to modify the value of the Prandtl number through the formula

$$
\frac{2}{3} \leq \operatorname{Pr}=\frac{1}{1-\nu} \leq+\infty
$$


Especially, $\operatorname{Pr}=2 / 3$ corresponds to $\nu=-1 / 2$ and (2.13) gives rise to a non convex combination. For this reason it was expected that the ES-BGK model is not entropic i. e. that (2.3) does not hold. In [2] it was proved that, unexpectedly, the H-theorem is satisfied and (2.3) holds true even for such negative values of $\nu$.

\subsection{The polyatomic ES-BGK model}

The extension to polyatomic gases can be performed using an internal energy parameter $I$ which takes into account the (continuous) internal degrees of freedom in a general way. This kind of formalism has been widely used, see [8], [14], [16]. The polyatomic distribution function $f(t, \underline{x}, \underline{v}, I)$ describes the number of particles with position $\underline{x}$, velocity $\underline{v}$ and internal energy $\epsilon(I)=I^{2 / \delta}$ at time $t$, and defines the macroscopic quantities $(2.6),(2.7),(2.11)$ by

$$
\begin{gathered}
\rho(\underline{x}, t)=<<f>>, \\
\underline{u}(\underline{x}, t)=\frac{1}{\rho}<<\underline{v} f>>, \\
E(\underline{x}, t)=<<\left(\frac{1}{2}|v|^{2}+I^{2 / \delta}\right) f>>=\frac{1}{2} \rho|u|^{2}+\rho e,
\end{gathered}
$$

under the new notation

$$
<<>>(\underline{x}, t)=\int_{\underline{v} \in R^{3}, I \in R^{+}} f(t, \underline{x}, \underline{v}, I) d \underline{v} d I .
$$

Here, the ratio of specific heats $\gamma=\frac{C_{p}}{C_{v}}$ and the number of additional degrees of freedom of the gas $\delta$ are related by

$$
\gamma=\frac{\delta+5}{\delta+3}
$$

By example, for a diatomic gas, we have $\delta=2$ and thus $\gamma=1.4$ is a usual value.

The specific internal energy $e=\frac{1}{\rho} E-\frac{1}{2}|u|^{2}$ can be divided here in two parts, the internal energy of translational motion $e_{t r}$ and the energy associated with the internal structure $e_{\text {int }}$ :

$$
e=e_{t r}+e_{i n t}
$$


with

$$
e_{t r}=\frac{1}{\rho}<<\frac{1}{2}|\underline{c}|^{2} f>>, \quad e_{i n t}=\frac{1}{\rho}<<I^{2 / \delta} f>>.
$$

We associate to these energies the corresponding temperatures $T_{e q}, T_{t r}, T_{\text {int }}$ by

$$
e=\frac{3+\delta}{2} R T_{e q}, \quad e_{t r}=\frac{3}{2} R T_{t r}, \quad e_{i n t}=\frac{\delta}{2} R T_{i n t} .
$$

As before, we also introduce pressure and the opposite of the stress tensor

$$
\begin{gathered}
P=\rho R T_{e q}, \\
\rho \underline{\underline{\Theta}}=<<\underline{c} \otimes \underline{c} f>>.
\end{gathered}
$$

Next, we have to introduce two relaxation parameters $0 \leq \theta \leq 1$ and $-\frac{1}{2} \leq \nu<1$. We define a relaxation temperature

$$
T_{r e l}=\theta T_{e q}+(1-\theta) T_{i n t},
$$

a corrected tensor

$$
\underline{\underline{\mathcal{T}}}=(1-\theta)\left((1-\nu) R T_{t r} \underline{\underline{I d}}+\nu \underline{\underline{\Theta}}\right)+\theta R T_{e q} \underline{\underline{I d}},
$$

and the generalized Gaussian

$$
\tilde{\mathcal{G}}[f]=\frac{\rho \Lambda_{\delta}}{\sqrt{\operatorname{det}(2 \pi \underline{\mathcal{T}})}\left(R T_{r e l}^{\delta / 2}\right)} \exp \left(-\frac{1}{2} \underline{c} \cdot \underline{\underline{\mathcal{T}}}^{-1} \cdot \underline{c}+\frac{I^{2 / \delta}}{R T_{r e l}}\right)
$$

We are now able to define the Gaussian-BGK polyatomic collision model

$$
Q(f)=\frac{P}{\mu(1-\nu+\theta \nu)}(\tilde{\mathcal{G}}[f]-f) .
$$

Here, the constant $\Lambda_{\delta}$ is defined by

$$
\Lambda_{\delta}^{-1}=\int e^{-I^{2} / \delta} d I
$$

so that

$$
<<\tilde{\mathcal{G}}[f]>>=<<f>>\text {. }
$$

The parameters $0 \leq \theta \leq 1$ and $-\frac{1}{2} \leq \nu<1$ can be chosen to obtain the correct 
Prandtl number of the flow and the second viscosity through the formulas (whose derivation is explained in [2])

$$
\frac{2}{3} \leq \operatorname{Pr}=\frac{1}{1-\nu+\theta \nu}<+\infty
$$

and

$$
\alpha=(\gamma-1)-\frac{1-\theta}{\theta}(1-\nu)\left(\frac{5}{3}-\gamma\right)
$$

\section{The numerical method}

We briefly describe the simulation of Boltzmann and ES-BGK equations (notice that BGK is a special case of the ES-BGK with $\nu=0$ ). They only differ in the collision step, especially in the strategy for computing collision frequencies. These are detailed in the next subsections.

\subsection{The random particle method for solving Boltzmann equation}

Here we present the numerical method used to compute the Boltzmann equation around a body. It is a variant of the classical DSMC method (Direct Simulation Monte-Carlo, see Bird [6], also [13], [22]), based on recent development as those of Babovsky [4].

It is a random particle method using identical particles. Each particle has a position, a velocity and an internal energy, denoted $\left(x_{i}, v_{i}, I_{i}\right)$, and at each time step the distribution function is approximated by

$$
f(x, v, I)=\frac{1}{N_{t}} \sum_{i=1}^{N_{t}} \delta\left(x-x_{i}\right) \delta\left(v-v_{i}\right) \delta\left(I-I_{i}\right), \quad 1 \leq i \leq N_{t}
$$

with $N_{t}$ the total number of particles.

In order to simulate collisions and to compute macroscopic quantities, we use a uniform rectangular mesh for the space variable $x$. For each time step, we perform a splitting of the Boltzmann equation into a free transport step and a collision step. The convergence of this fractional step method has been proved by L. Desvillettes and S. Mischler [20] for the BGK equation. During the free transport step, a particle with position $x_{i}$ and velocity $v_{i}$ is transported to the position $x_{i}+\Delta t v_{i}$, and reflections around the body are preformed. 
The collision step consists, for each cell, in changing velocities and internal energies of particles of the cell by simulated binary collisions. For this, we randomly couple the $N$ particles of the cell and for each pair $\left(v_{i}, v_{j}\right)$, we decide if a collision occurs according to the probability

$$
q_{i j}=\Delta t \frac{N-1}{N_{\infty}} \frac{2^{\omega-0.5}}{\sqrt{2} \Gamma\left(\frac{5}{2}-\omega\right)}\left|v_{i}-v_{j}\right|^{2(1-\omega)} .
$$

Here $\frac{N}{N_{\infty}}$ represents the cell density, and $\omega$ is the exponent of the viscosity law $\mu=\mu_{\infty}\left(\frac{T_{t r}}{T_{\infty}}\right)^{\omega}, \frac{1}{2} \leq \omega \leq 1$ ( $\omega=\frac{1}{2}$ corresponds to hard spheres collisions).

To decide if a collision occurs or not, we choose randomly a value $a$ in $(0,1)$ uniformly distributed and, if $a>q_{i j}$ the collision does not occur, if $a<q_{i j}$, we perform a collision. We compute velocities and internal energies after collision with a model which conserves momentum and total energy $\left(\frac{1}{2}\left|v_{i}\right|^{2}+I_{i}\right)$ of the incident particles. In the polyatomic case, exchange between translational and rotational energies is obtained with the Larsen-Borgnakke model. In order to obtain the right relaxation of internal energy, a relaxation collision number $Z_{\text {rot }}$ is chosen (for nitrogen $Z_{\text {rot }}=$ $5)$. With a probability $\frac{1}{Z_{\text {rot }}}$ we exchange internal energy and translational energy (inelastic collisions), else we exchange only translational energy (elastic collisions). To take into account external boundary conditions, we inject particles via a layer of boundary cells in which particles have velocities and internal energies distributed following a Maxwellian function $M\left(\rho_{\infty}, u_{\infty}, T_{\infty}\right)$.

The cell size is chosen less than the free stream mean free path, as far as possible. The hydrodynamical time step is chosen so that a particle is transported at most in a neighbouring cell. For collisions, we need to divide this time step in order to obtain collision probabilities less than 1 in (3.1) and hence we need to compute several cyles of collision.

\subsection{Random method for the ES-BGK model}

We only describe here the collision step since it is the only difference with the random particle method for solving Boltzmann equation. We present the most general case: polyatomic gas, variable hard spheres collisions. At the time step $n$, after free transport, we have in each cell in the physical space a set of particles with velocities and internal energies $\left(v_{i}, I_{i}\right)_{1 \leq i \leq N}$, where $N$ is the number of particles in the cell, and we want to compute $\left(v_{i}^{n+1}, I_{i}^{n+1}\right)_{1 \leq i \leq N}$. 


\subsubsection{Collision probability of a cell}

Here, in opposition to the binary collisions of Boltzmann equation, either we change velocities and internal energies of all particles of the cell, or none of the particles have collision. This is because the collision probability law is defined with macroscopic quantities.

Practically we compute in each cell mean velocity, translational temperature, rotational temperature and equilibrium temperature by

$$
\begin{aligned}
& u=\frac{1}{N} \sum_{i=1}^{N} v_{i} \\
& R T_{t r}=\frac{1}{3 N} \sum_{i=1}^{N}\left|v_{i}-u\right|^{2} \\
& R T_{\text {rot }}=\frac{2}{\delta N} \sum_{i=1}^{N} I_{i} \\
& T_{e q}=\frac{3 T_{t r}+\delta \bar{T}_{r o t}}{3+\delta}
\end{aligned}
$$

where $\delta$ is the number of internal degrees of freedom of the gas.

Next, we introduce the collision probability of the cell

$$
\operatorname{Proba}=\Delta t \frac{N}{N_{\infty}} R T_{t r} \frac{P r}{\mu}
$$

with

$$
\begin{aligned}
& \frac{N}{N_{\infty}} \text { the cell density, } \\
& \operatorname{Pr}=\frac{1}{1-\nu+\theta \nu} \text { the Prandtl number, } \\
& \mu=\mu_{\infty}\left(\frac{T_{t r}}{T_{\infty}}\right)^{\omega} \text { the viscosity. }
\end{aligned}
$$

Then, we decide with a random test if the cell is a collisional cell or not. For this, we choose a random number $a_{2} \in(0,1)$ uniformly distributed and, if $a_{2}<$ Proba, we perform a collision of all the cell particles, else we keep the quantities $v_{i}^{n}$ and $I_{i}^{n}$. 


\subsubsection{Gaussian collision of the cell particles}

In the case of a collisional cell $\left(a_{2}<\right.$ Proba), we compute the tensor $\underline{\underline{\Theta}}$ by

$$
\Theta_{k \ell}=\frac{1}{N} \sum_{i=1}^{N}\left(v_{i}^{k}-u^{k}\right)\left(v_{i}^{\ell}-u^{\ell}\right), \quad 1 \leq k, \ell \leq 3,
$$

and then we compute the corrected tensor

$$
\underline{\underline{\tau}}=(1-\theta)\left[(1-\nu) R T_{t r} \underline{\underline{\boldsymbol{I d}}}+\nu \underline{\underline{\Theta}}\right]+\theta R T_{e q} \underline{\underline{\boldsymbol{I d}}}
$$

with the parameters $\nu, \theta$ chosen according to the Prandtl number and second viscosity (see formulas $2.27,2.28$ ). Notice that

$$
-\frac{1}{2} \leq \nu<1 \quad \text { and } \quad 0 \leq \theta \leq 1
$$

Then, we generate the new values $\left(v_{i}^{n+1}, I_{i}^{n+1}\right)_{1 \leq i \leq N}$ according to the gaussian distribution

$$
\frac{\rho \Lambda_{\delta}}{\sqrt{\operatorname{det}(2 \pi \underline{\underline{T}})}\left(R T_{r e l}^{\delta / 2}\right)} \exp \left(-\frac{1}{2}(\underline{v}-\underline{u}) \cdot \underline{\underline{T}}^{-1} \cdot(\underline{v}-\underline{u})+\frac{I^{2 / \delta}}{R T_{r e l}}\right)
$$

where the relaxation temperature $T_{r e l}$ is defined by

$$
T_{\text {rel }}=\theta T_{e q}+(1-\theta) T_{\text {rot }}
$$

\section{First step. Generation of velocities}

We generate $\left(w_{i}\right)_{1 \leq i \leq N}$ according to the centered and normalized maxwellian distribution

$$
\frac{1}{(2 \pi)^{3 / 2}} \exp \left\{-\frac{1}{2}|w|^{2}\right\}
$$

which gives $w_{i}^{x}=\sqrt{-2 \ln b_{1}} \cos 2 \pi b_{2}, w_{i}^{y}=\sqrt{-2 \ln b_{1}} \sin 2 \pi b_{2}, w_{i}^{z}=\sqrt{-2 \ln b_{3}} \cos 2 \pi b_{4}$, where $b_{1}, b_{2}, b_{3}, b_{4}$ are random numbers uniformly distributed between 0 and 1 . The new velocities are obtained by the formula

$$
v_{i}=u+\underline{\underline{\mathcal{T}}}^{1 / 2} \cdot w_{i}
$$




\section{Second step. Generation of internal energies}

In the polyatomic case, internal energies $\left(I_{i}\right)_{1 \leq i \leq N}$ are generated by

$$
I_{i}=-\frac{\delta}{2} R T_{r e l} \ln \alpha_{i}
$$

where $\alpha_{i}$ are random numbers uniformly distributed between 0 and 1 .

\section{Third step. Correction to assume conservation laws}

To enforce the conservation laws, which are fundamental quantities in the hydrodynamical limit, see [2], we conserve momentum by introducing $\tilde{w}_{i}$, with mean value zero, defined by

$$
\tilde{w}_{i}=w_{i}-\frac{1}{N} \sum_{i=1}^{N} w_{i} .
$$

Next we impose the conservation of total energy using vectors $\lambda \underline{\underline{\tau}}^{1 / 2} \cdot \tilde{w}_{i}$ obtained with

$$
\lambda=\left(\frac{\frac{3+\delta}{2} R T_{e q}-\frac{1}{N} \sum_{i=1}^{N} I_{i}}{\frac{1}{2 N} \sum_{i=1}^{N}\left|\underline{\underline{\tau}}^{1 / 2} \cdot \tilde{w}_{i}\right|^{2}}\right)^{1 / 2} .
$$

Finally the new velocities are

$$
v_{i}=u+\lambda \underline{\underline{\tau}}^{1 / 2} \cdot \tilde{w}_{i}
$$

Remark 3.1 The symmetric matrix $\underline{\underline{\tau}}^{1 / 2}$ is computed with the help of eigenvalues and eigenvectors of $\underline{\underline{\tau}}$, according to the formula

$$
\underline{\mathcal{T}}_{i j}^{1 / 2}=\sum_{k=1}^{3} \operatorname{vec}(i, k) \cdot \sqrt{\operatorname{val}(k)} \cdot \operatorname{vec}(j, k) .
$$

Remark 3.2 The BGK model and monoatomic ES-BGK model can be deduced from the polyatomic ES-BGK model:

$\nu=0$ or $\theta=1$ corresponds to the $B G K$ model. Hence $\operatorname{Pr}=1$,

$\theta=0$ corresponds to the monoatomic ES-BGK model, $\operatorname{Pr}=\frac{1}{1-\nu}$. 
Remark 3.3 As for the Boltzmann collision operator, it is natural to choose for $\theta$ the inverse of the relaxation collision number $Z_{\text {rot }}$ to exchange translational and rotational energies.

$$
\theta=\frac{1}{Z_{\text {rot }}}
$$

Hence, for nitrogen $\theta=0.2$. But $\theta=0.4$, which corresponds to $\operatorname{Pr}=0.77$ seems better.

\section{Numerical comparison between ES-BGK and Boltz- mann models}

The comparison tests concern semi-rarefied monoatomic flows around a plate $\left(K n_{\infty}=\right.$ $510^{-3}$ and $1.4410^{-2}$, Mach $\left._{\infty}=18.3\right)$ and a near-continuum diatomic flow over a ramp with a flap deflection of 25 degrees $\left(K n_{\infty}=610^{-4}\right.$, Mach $\left._{\infty}=10\right)$.

Experimental results around a plate obtained by Allegre and al [1] have inspired our tests for a plate. In the ramp case, experimental results were obtained by Chanetz and al [10] and a precise Monte-Carlo simulation of the Boltzmann equation was computed by Ivanov and al. [12], allowing to validate our simulations.

\subsection{The plate test case}

It is a simple case (monoatomic gas, hard spheres collision) in order to perform a first comparison of the ES-BGK, BGK and Boltzmann models.

\subsubsection{Plate data}

We consider a semi-rarefied flow around a model plate of $10 \mathrm{~cm}$ length, without incidence.

$10 \mathrm{~cm}$

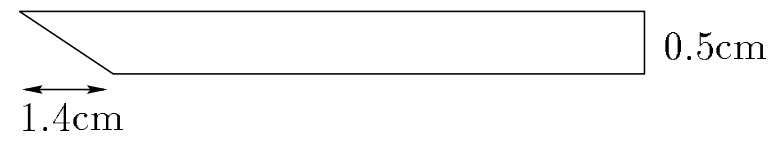

$\mathrm{RR} \mathrm{n}^{\circ} 3872$ 
The gas is monoatomic with $R=297$. The free stream conditions are: $\lambda_{\infty}=$ $510^{-4} \mathrm{~m}$ (hence $K n_{\infty}=510^{-3}$ ), $\rho_{\infty}=5.610^{-5} \mathrm{~kg} / \mathrm{m}^{3}, T_{\infty}=13.6 \mathrm{~K}, V_{\infty}=$ $1503 \mathrm{~m} / \mathrm{s}$, from which we deduce Mach $_{\infty}=18.33$

Diffuse reflection model with complete accomodation is used for wall reflection with a wall temperature $T_{W}=286 \mathrm{~K}$.

For the viscosity law : $\mu=\mu_{\infty}\left(\frac{T}{T_{\infty}}\right)^{\omega}$ we choose $\omega=0.5$ (hard sphere model) and $\mu_{\infty}=0.12510^{-5}$ Pa.s (hence $R e_{\infty}=604$ ).

The Prandtl number is $\operatorname{Pr}=\frac{2}{3}$ for the ES-BGK model $\left(\nu=-\frac{1}{2}, \theta=0\right)$ and $\operatorname{Pr}=1$ for the BGK model. The computational domain is a rectangle of size : $0.116 \mathrm{~m} \times 0.07 \mathrm{~m}$, meshed with rectangles of size : $h_{x}=h_{z}=510^{-4} \mathrm{~m}$. The time step is : $\Delta t=2.8210^{-7} \mathrm{~s}$. Macroscopic variables are computed over 1000 time steps after 1500 time steps to reach a stationary state. A free stream cell contains 25 particles.

\subsubsection{Results of the plate test case for $K n=510^{-3}$}

Results of this test case are presented on figures $1, \ldots, 14$.

Globally, results of ES-BGK model are not far from those of Boltzmann equation, except for the shock of the Mach number whose junction with the free stream zone is too diffusive. This is slightly visible on the temperature where only the relative error would be significantly large, but it is amplified on the Mach number because $\sqrt{T}$ is small in this zone, and we have the formula $M a c h=\frac{|u|}{\sqrt{\gamma R T}}$.

For the wall coefficients, the difference is only visible at the beginning of the plate, especially on the lower part. The global aerodynamic coefficients, drag, lift and heat, are less than $5 \%$ different

$$
\begin{array}{llll}
\text { ES-BGK } & C_{D}=0.0934 & C_{L}=0.0750 & C_{H}=-0.0286 \\
\text { Boltzmann } & C_{D}=0.0975 & C_{L}=0.0775 & C_{H}=-0.0303
\end{array}
$$

Comparison between $\operatorname{Pr}=\frac{2}{3}$ (continue line) and $\operatorname{Pr}=1$ (large dotted line) is presented on figures $7, \ldots, 14$.

We observe that $\operatorname{Pr}=\frac{2}{3}$ is clearly better than $\operatorname{Pr}=1$ in the boundary layer (fig. 7 and 8 ), especially for the temperature whose maximum value is too high in the case $\operatorname{Pr}=1$.

For the wall coefficients $C_{p}, C_{f}, C_{h}$ (pressure, skin friction, heat flux coefficients) presented on figures $7,8,9, \operatorname{Pr}=\frac{2}{3}$ and $\operatorname{Pr}=1$ give both results close to Boltzmann results. 


\subsubsection{Plate results for $K n=1.4410^{-2}$}

We are interested here by the behaviour of the three models when the Knudsen number $K n_{\infty}$ is increasing. The data are the same except that $\lambda_{\infty}=1.4410^{-3}$, from which we deduce $K n_{\infty}=1.4410^{-2}$.

The results are presented on figures $15, \ldots, 26$.

Globally, ES-BGK results are still not too far from Boltzmann results, especially near the wall, but the differences are more important.

The results obtained with the BGK model are still different of those obtained with the ES-BGK model in the boundary layer, but not more than for smaller Knudsen number, especially for temperature (fig. 20). It seems that this difference is not proportional to the Knudsen number. On the other hand, the connexion of the Mach shock with free stream is worst and linked to the knudsen number. We will later see that it disappears for the ramp when $K n_{\infty} \sim 10^{-3}$.

\subsection{The ramp test case}

We compare here the ES-BGK and Boltzmann models in a more realistic diatomic case.

\subsubsection{Ramp data}

We consider a concave body consisting of a plate extended by a ramp at a 25 degrees angle, in a cold near continuum flow, without incidence. This test is difficult because of a shock-boundary layer interaction and a recirculation zone which requires a very long computational time to establish a stationary regime. The test is performed with Mach 10.

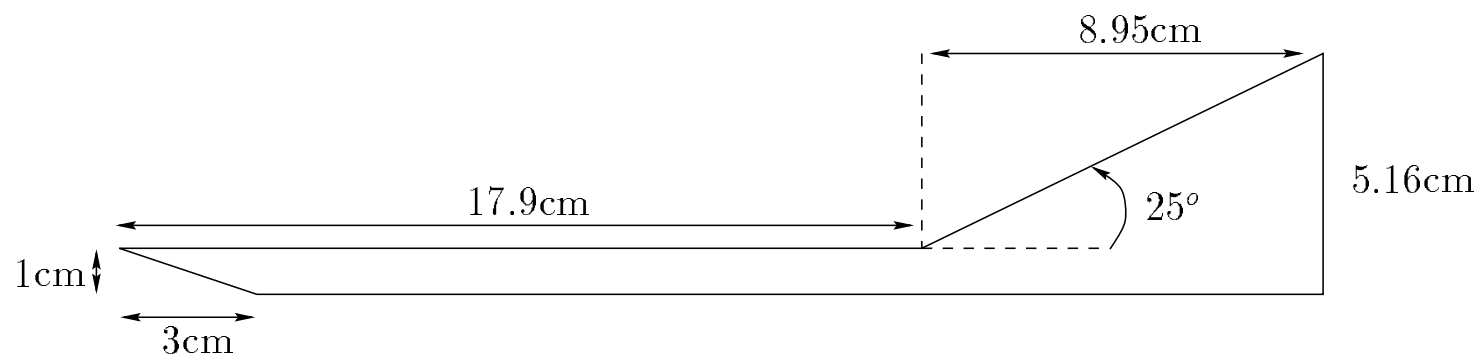


The gas is nitrogen with $R=297$. The free stream conditions are: $\lambda_{\infty}=$ $1.0710^{-4} \mathrm{~m}$, (hence $K n_{\infty}=610^{-4}$ for a characteristic length $L=0.179 \mathrm{~m}$ which corresponds to the length of the flat part of the body), $\rho_{\infty}=3.910^{-4} \mathrm{~kg} / \mathrm{m}^{3}, T_{\infty}=$ $52 \mathrm{~K}, V_{\infty}=1477 \mathrm{~m} / \mathrm{s}$, hence Mach $_{\infty}=10$.

The wall temperature is $T_{W}=290 \mathrm{~K}$, and the the wall reflection of particles is modelized by the diffuse reflection model with total accomodation.

For the viscosity law $: \mu=\mu_{\infty}\left(\frac{T_{t r}}{T_{\infty}}\right)^{\omega}$, we choose $\omega=0.75$ and $\mu_{\infty}=0.50710^{-5}$ Pa.s. Hence $R e_{\infty}=20300$, based on the total length of the body : $26.85 \mathrm{~cm}$.

The two parameters of the diatomic ES-BGK model are $\nu=-0.5, \theta=0.4$, hence $\operatorname{Pr}=0.77$. Size of the computational domain is : $0.31 \mathrm{~m} \times 0.09 \mathrm{~m}$. It is meshed with rectangular cells with size $h_{x}=210^{-3} \mathrm{~m}, h_{z}=510^{-4} \mathrm{~m}$. The time step is $\Delta t=410^{-7} \mathrm{~s}$. Macroscopic quantitites are computed over 5000 time steps after 10000 time steps to obtain the steady solution. A free stream cell contains 25 particles.

\subsubsection{Results of the ramp test case}

We present here results obtained with ES-BGK, Boltzmann and BGK models for the ramp test case. The ES-BGK and Boltzmann solutions are very close, especially the density, the Mach number and the translational temperature; this is true both in the boundary layer and in the shock (fig.29, 30, 33, 34, 37, 38).

Wall coefficients and tangential velocity are nearly coinciding (fig. $41, \ldots, 44$ ).

The parameter $\theta$ adjusts exchange between internal and translational energy. The choice $\nu=-0.5, \theta=0.4$ which corresponds to $\operatorname{Pr}=0.77$ seems to be the best.

We note that the BGK solution is not so good in the boundary layer than the ESBGK solution, in particular for the density(fig.30), the global temperature(fig.31,32) and the translational temperature(fig. 37,38 ).

The small value of the Knudsen number, $K n=610^{-4}$ explains the good agreement between ES-BGK and Boltzmann solutions. In fact, the beginning of the ramp can be considered as a plate with a Knudsen number $K n=10^{-3}$ (with the characteristic length of the plate), and we observe in this case a good connexion between the Mach shock and the free stream, in opposition to the plate with $K n=510^{-3}$.

Hence, for a near continuum flow, with recirculation zone and shock-boundary layer interaction, the ES-BGK model gives nearly the the same results as a Boltzmann simulation. Moreover, the computational time is $30 \%$ smaller (21h against $30 \mathrm{~h}$ on a HP 9000 station (PA 8200, 240Mhz)) because the collision step for Boltzmann equation needs subcyles to obtain a correct probability of collision. 


\section{Study of the velocity distribution function}

The cases studied above show clearly a good agreement between the ES-BGK and Boltzmann simulations. A limitation of these results is that rarefaction effects should be quite small, except at the boundary. In such a situation one can expect the distribution functions to be close to a maxwellian equilibrium in most of the flow, since the Boltzmann and ES-BGK models have the same Chapmann Enskog expansion, the agreement of the solutions follows naturally.

To put evidence on this, we conducted a more accurate study of the velocity distributions in different cells, with a special interest in the cells in the boundary layer and in the shock layer, where desequilibrium can be expected. Such a study is close to that of Aoki and al.[3] for a thin plate, with the BGK model.

As before we study the flow around a plate, for a monoatomic Hard Sphere gas.

To compute the velocity distribution in a given cell, during the time steps used for averaging, the program sorts the velocities in the cell inside a fine velocity grid. Here we consider a $2 \mathrm{D}$ flow, so that we study the velocities $v_{x}$ and $v_{z}$, the cell in velocity is of a width of $20 \mathrm{~m} . \mathrm{s}^{-1}$, and the velocities are supposed to be between $-1900 \mathrm{~m} . \mathrm{s}^{-1}$ and $2100 \mathrm{~m} . \mathrm{s}^{-1}$ (the infinite velocity being $u_{\infty}=1502 \mathrm{~m} . \mathrm{s}^{-1}$ ). Thus there are $200 \times 200$ velocity cells.

At each time step, if a particle has a velocity which fits into a velocity cell, then the counter of that cell increases by one, at the end we divide all such numbers by the total number of particles for all the time steps.

Then we draw the $2 \mathrm{D}$ velocity distribution, for example we can represent the distribution function isovalues.

This study can be performed in different spatial cells (see figure below), and we consider different situations: a cell(4) before the shock layer, a cell(3) inside the shock layer and two cells (1 and 2) in the boundary layer, in the case of a simulation of the Boltzmann equation.

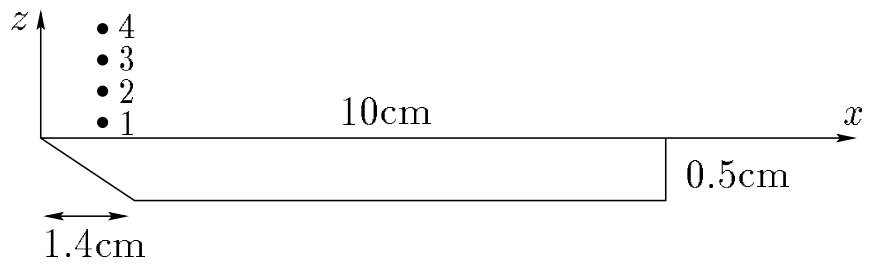

$\mathrm{RR} \mathrm{n}^{\circ} 3872$ 
Before the shock, the flow is mainly at equilibrium, velocities and temperatures are $u_{\infty}, T_{\infty}$, the velocity distribution is close to a maxwellian, for the $2 \mathrm{D}$ isovalues, we thus observe circles, and the radius is quite small (see fig.45). The variance of the distribution being the square root of $T_{\infty}$, it is quite small, and in $3 \mathrm{D}$ the distribution is close to a Dirac. Then, as the cell gets inside the shock layer, the distribution "spreads" (as the temperature increases). A remarkable fact is that thought the isovalues are close to circles, it is no more isotropic, mainly the distribution spreads specificially more in one direction (see figure 45).

Near the wall, the distribution differs completely from a maxwellian (see figure 46). Compared to a maxwellian, the distribution is spread unevenly, it looks a lot like a two hilltop distribution. To understand it better, we can consider one dimensional cuts, for example the distribution of $v_{x}$. It is obtained by integrating over $v_{z}$. In particular one can still observe the peak corresponding to the flow at infinity (particles which have not yet collided, this corresponds to a peak around the $1500 \mathrm{~m} . \mathrm{s}^{-1}$ velocity), mixed with the velocities given by the wall condition (in our case particles colliding are reemitted on a maxwellian with zero velocity and temperature $\left.T_{\text {wall }}\right)$. This effect is still observed with a good agreement in the ESBGK distribution, but is not at all taken into account by a BGK simulation where the peak corresponding to the flow at infinity has disappeared (see figures 47 to 50 ). The explanation seems to be that the ES-BGK model has a lower collision rate than the BGK model, the ratio between the two being the one of Prandtl numbers. A BGK simulation with this smaller collision rate give also a distribution taking correctly into account particles wich have not yet collided.

\section{Conclusion}

The Monte Carlo simulation of the ES-BGK equation performed in realistic conditions gives results in good agreement with the Boltzmann equation especially for $K n<10^{-2}$. In strong desequilibrium region, the ES-BGK model performs better than the usual BGK model. This can be noticed in the boundary layer where both the distribution functions and macroscopic quantities are better predicted using ES-BGK model than using BGK model. It is naturally explained by the correct transport coefficient obtained with the ES-BGK model.

Since the equation is simpler to solve, one can expect new schemes adapted to BGK type equation, allowing a resolution in cases where the usual DSMC gives no result. Especially one can expect deterministic schemes following the ideas of [19],[23]. 


\section{References}

[1] J. Allegre, M. Raffin, J.C. Lengrand, A. Chpoun, and L. Goettesdiener. Champs d'écoulements hypersoniques au voisinage d'une plaque plane. Rapport final de contrat $87 / 392$, DRET, Septembre 89.

[2] P. Andries, B. Perthame, P. Le Tallec, and J.P. Perlat. The Gaussian BGK model of Boltzmann equation with small Prandtl number. To appear in Eur. $J$. of Mech. B/ fluids.

[3] K. Aoki, K. Kanba, and S. Takata. Numerical analysis of a supersonic rarefied gas flow past a flat plate. Phys. Fluids, 9(4):1144-1161, 1997.

[4] H. Babovsky. On a simulation scheme for the Boltzmann equation. Math. Methods Appl. Sci., 8(2):223-233, 1986.

[5] P.L. Bhatnagar, E.P. Gross, and M. Krook. A model for collision processes in gases. Phys. Rev., 94:511, 1954.

[6] G.-A. Bird. Molecular Gas Dynamics and the Direct Simulation of Gas Flow. Oxford Engineering Science Series Publications, 1994.

[7] J.-F. Bourgat, L. Desvillettes, P. Le Tallec, and B. Perthame. Microreversible collisions for polyatomic gases and Boltzmann theorem. Eur. J. Mech. B/Fluids, 13(2):237-254, 1994.

[8] R. Brun. Transport et relaxation dans les écoulements gazeux. Masson, 1986.

[9] C. Cercignani. The Boltzmann Equation and its Applications. Applied Mathematical Sciences, Springer-Verlag., 1988.

[10] B. Chanetz, M.C. Coet, D. Nicout, and T. Pot. Shock wave-boundary layer interaction in hypersonic two dimensional laminar flow. In Colloque sur les écoulements hypersoniques, pages 656-661. ONERA, 5-7 octobre 1992.

[11] L. H. Holway. Kinetic theory of shock structure using an ellipsoidal distribution function. In Rarefied Gas Dynamics, Vol. I (Proc. Fourth Internat. Sympos., Univ. Toronto, 1964), pages 193-215. Academic Press, New York, 1966.

[12] M.S. Ivanov, G.N. Markelov, A.N. Kudriavtsev, and S.F. Gimelshein. Numerical investigation of shock wave and boundary layer interaction in the nearcontinuum hypersonic flow over concave bodies. In Computational Fluid Dynamics. 1996. 
P. Andries, J.F. Bourgat, P. Le Tallec, B. Perthame

[13] M.S. Ivanov and S.V. Rogasinsky. Analysis of numerical techniques of direct simulation Monte-Carlo method in the rarefied gas dynamics. J. Numer. Anal. Math. Modelling, 3(6):453-465, 1988.

[14] B. Khobalatte and B. Perthame. Maximum principle on the entropy and minimal limitations for kinetic schemes. Math. Comp., 17(205):119-131, 1994.

[15] P.S Larsen and C. Borgnakke. Statistical collision model for simulating polyatomic gas with restricted energy exchange. 9th International symposium on rarefied gas dynamics, Gottingen, 1974.

[16] E.M. Lifschitz and L.P. Pitaevski. Physical kinetics. Pergamon Press, Oxford, 1981.

[17] P.L. Lions. Compactness in Boltzmann equation via Fourier integral operators and applications. Part 1, 2 and Part 3. J. Math. Kyoto Univ., 34(2) and 34(3):161 and 539-584, 1994.

[18] S. K. Loyalka, Petrellis N., and T. S. Storvick. Some exact numerical results for the BGK model: Couette, Poiseuille and thermal creep flow between parallel plates. Z. Angew. Math. Phys., 30(3):514-521, 1979.

[19] L. Mieussens. Discrete velocity model and implicite scheme for the BGK equation of rarefied gas dynamics. Math. Models and Meth. Appl. Sci., to appear.

[20] S. Mischler and L. Desvillettes. About the splitting algorithm for Boltzmann and BGK equations. M3AS, 6:1079-1101, 1996.

[21] L. Pareschi, G. Russo, and R.E. Caflisch. Implicit monte carlo methods for rarefied gas dynamics. Preprint, Universita di Ferrara, (274).

[22] B. Perthame. Introduction to the theory of random particle methods for the Boltzmann equation. In World scientific, editor, Advances in kinetic theory and computing, volume 22. SAMAS, singapore, 1994.

[23] F. Rogier and J. Schneider. A direct method for solving the Boltzmann equation. Transport Theory Statist. Phys., 23(1-3):313-338, 1994. 


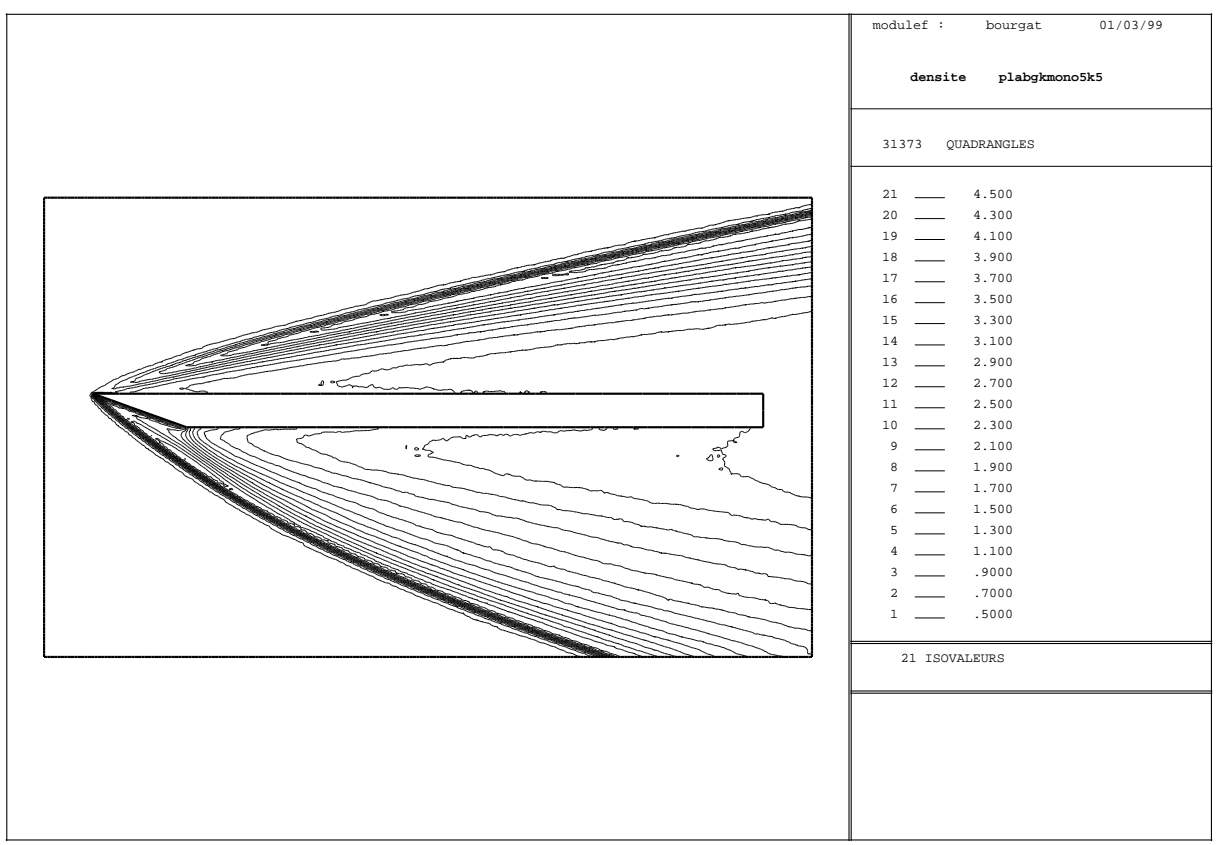

Figure 1: plate, $K n=510^{-3}$, ES-BGK isodensities

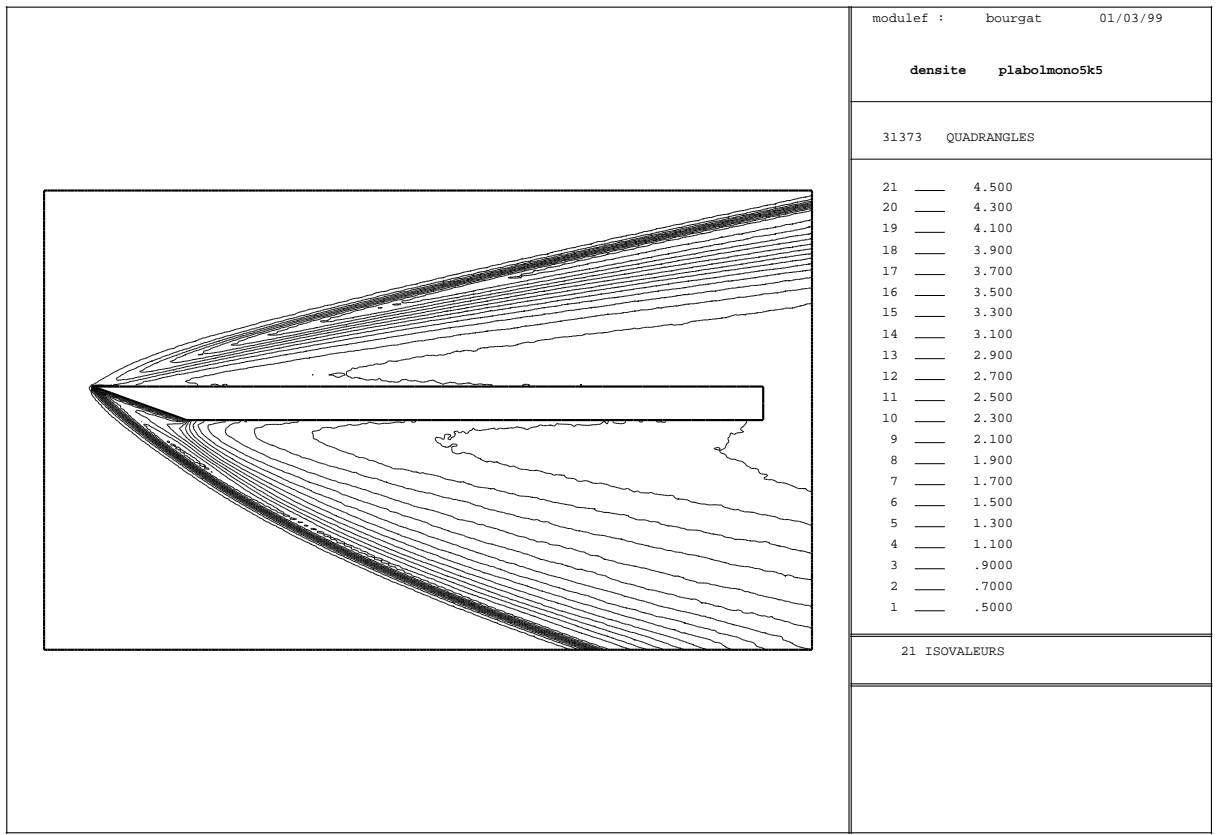

Figure 2: plate, $K n=510^{-3}$, Boltzmann isodensities

$\operatorname{RR} n^{\circ} 3872$ 


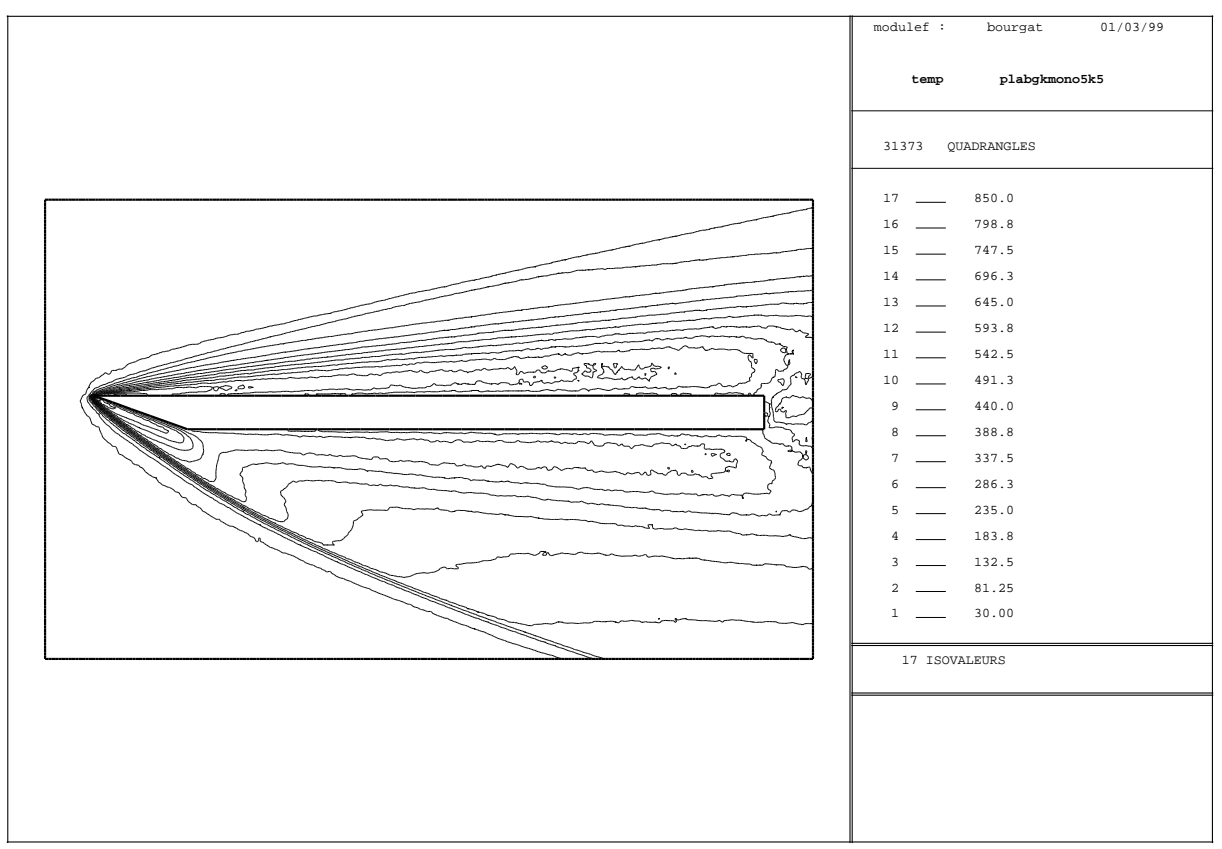

Figure 3: plate, $K n=510^{-3}$, ES-BGK isotemperatures

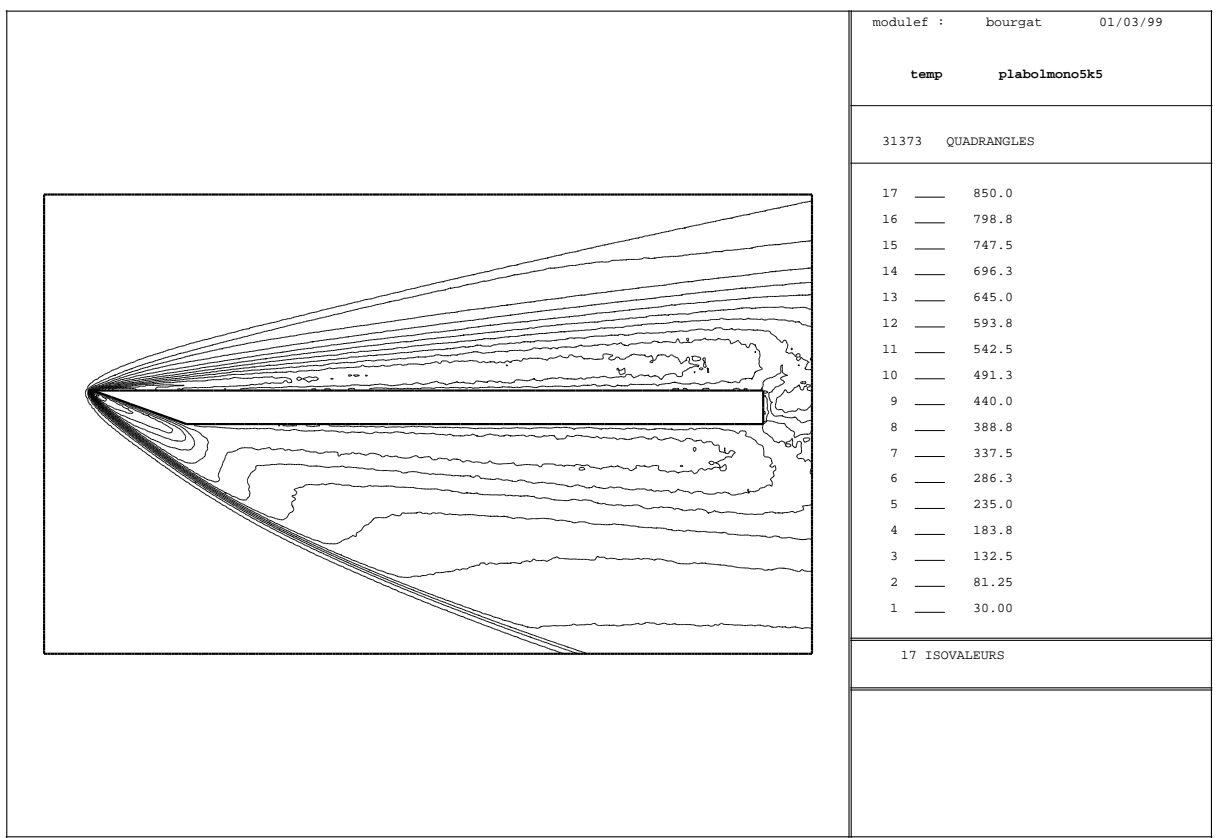

Figure 4: plate, $K n=510^{-3}$, Boltzmann isotemperatures 


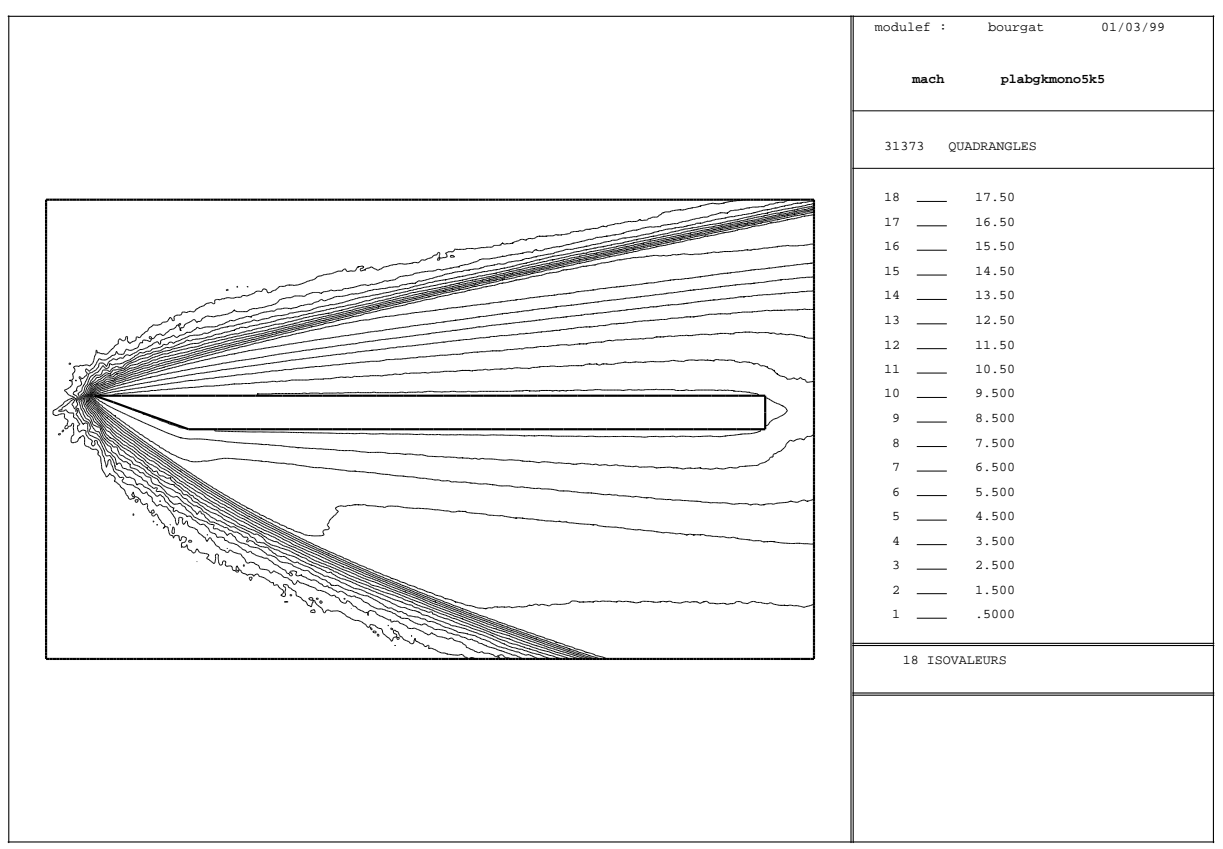

Figure 5: plate, $K n=510^{-3}$, ES-BGK isomachs

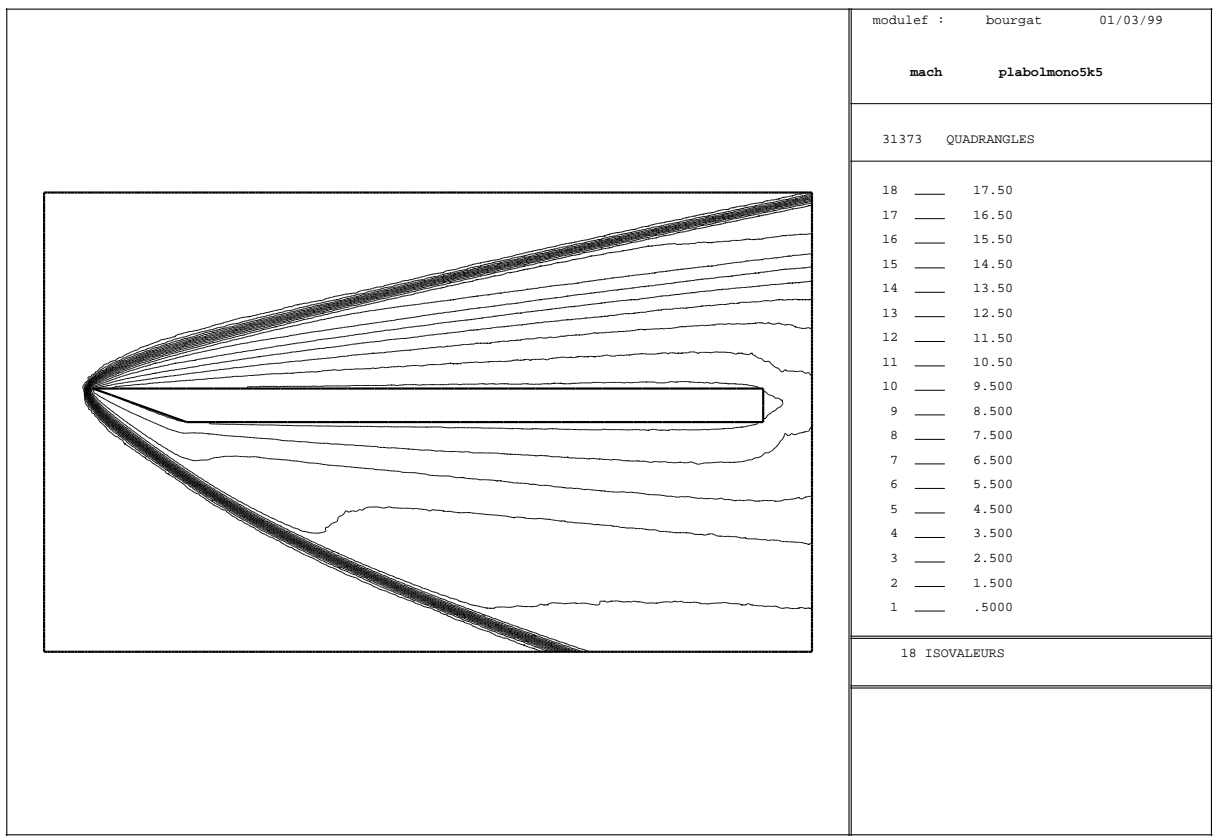

Figure 6: plate, $K n=510^{-3}$, Boltzmann isomachs

$\operatorname{RR} n^{\circ} 3872$ 


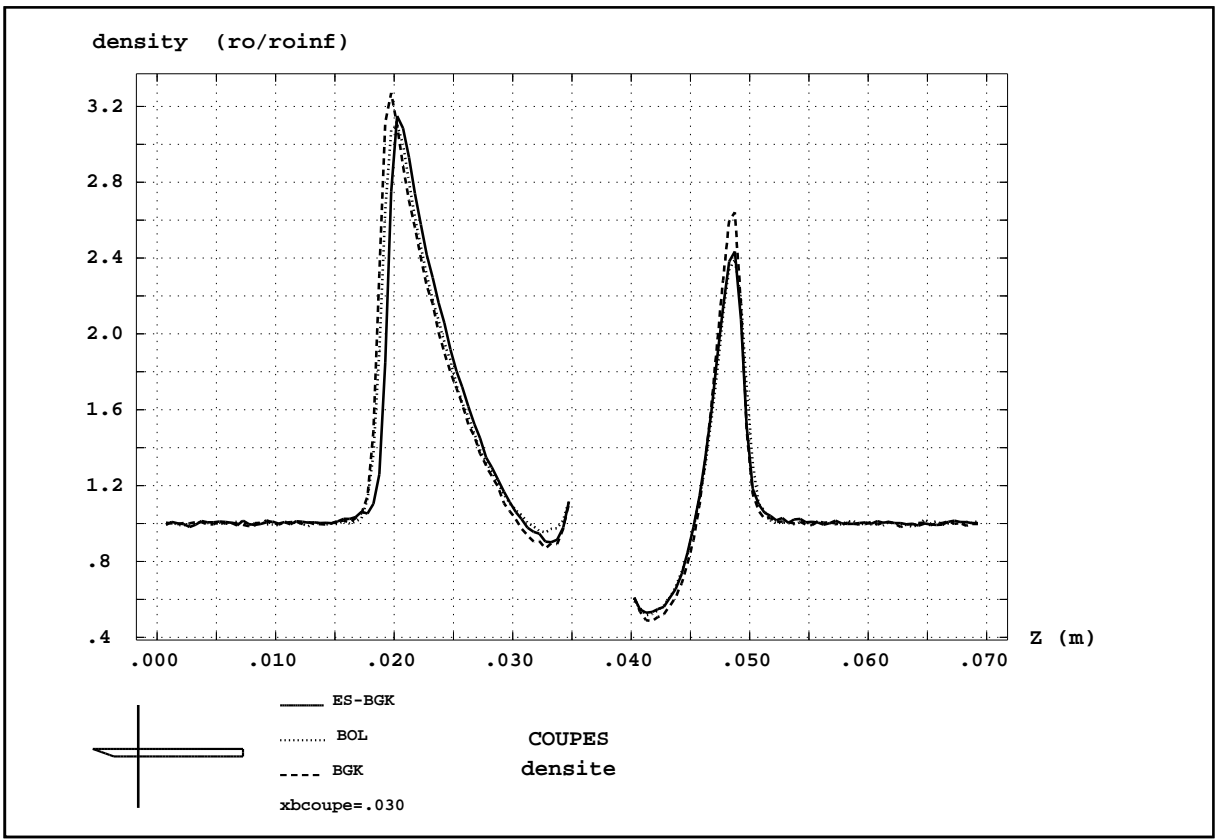

Figure 7: plate, $K n=510^{-3}$, cross section of the density at $\mathrm{x} / \mathrm{L}=0.3$

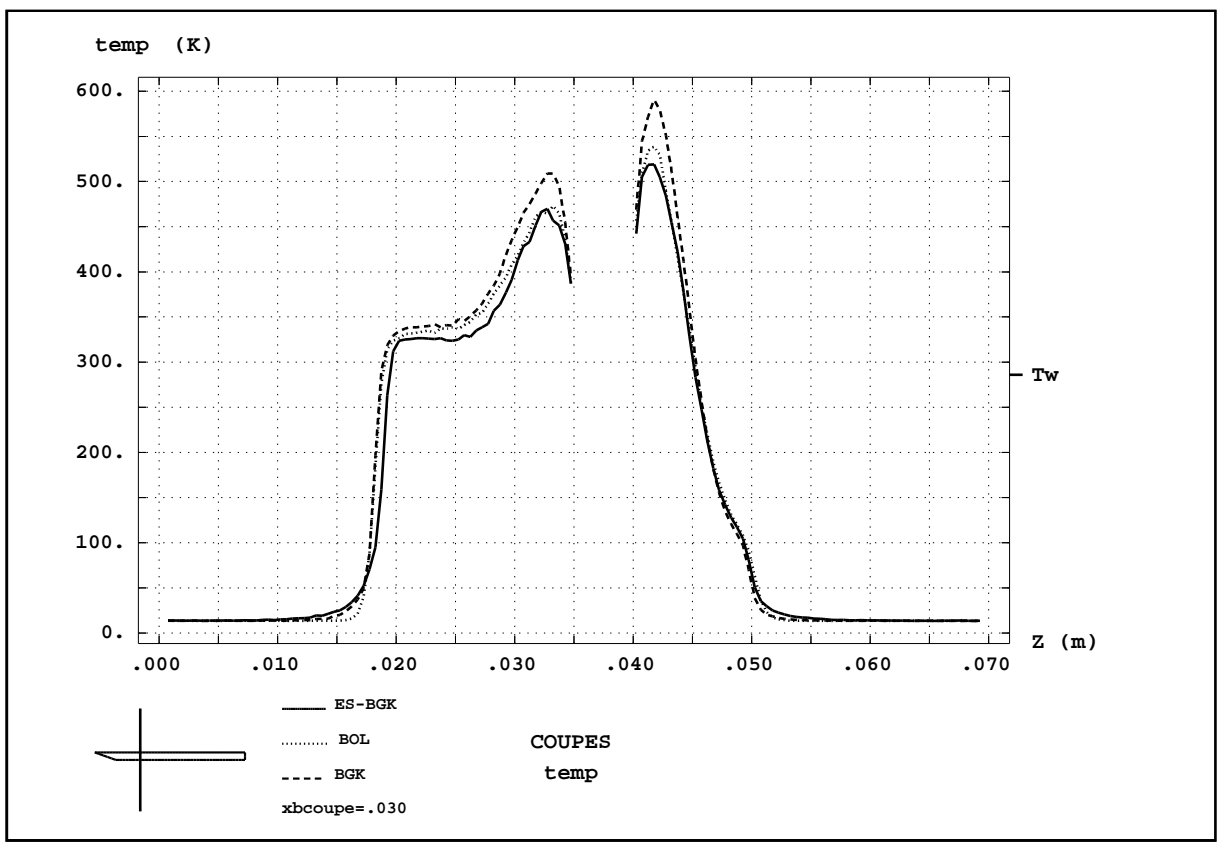

Figure 8: plate, $K n=510^{-3}$, cross section of the temperature at $\mathrm{x} / \mathrm{L}=0.3$ 


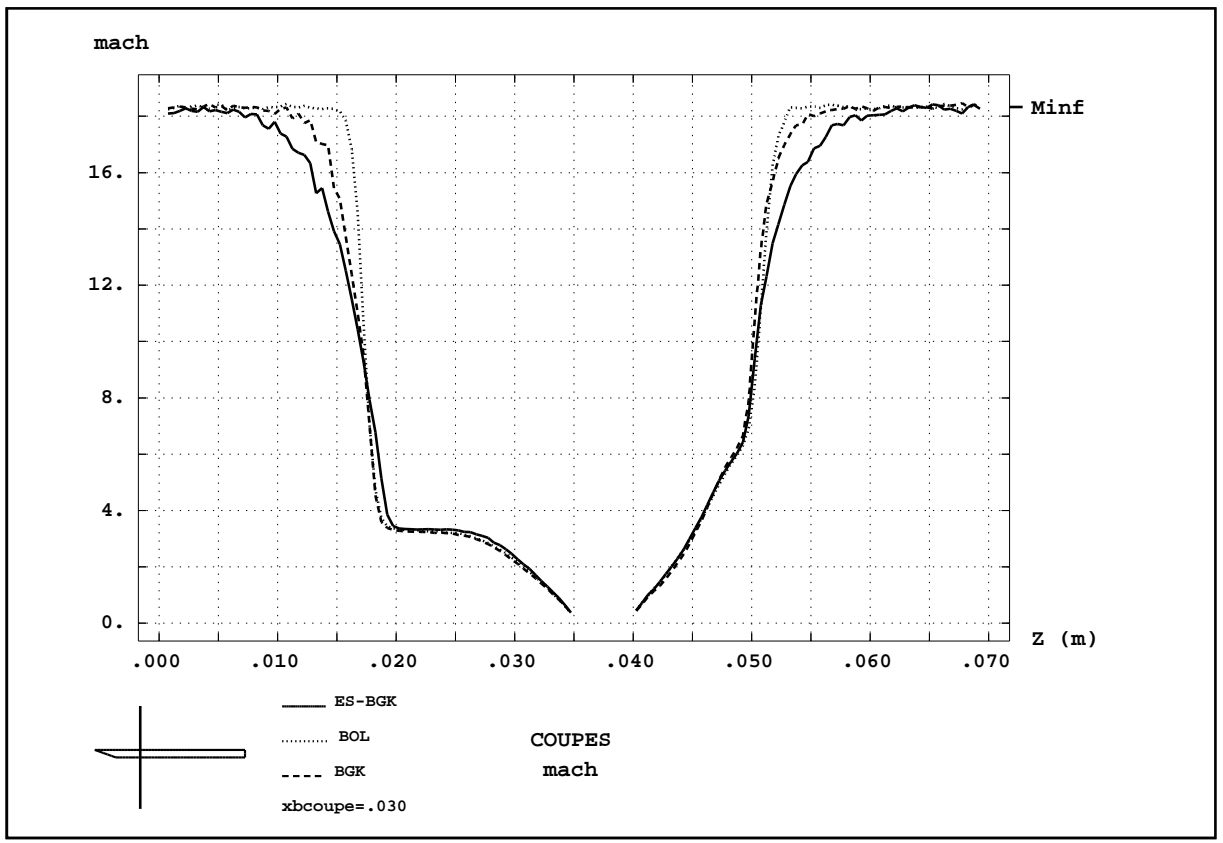

Figure 9: plate, $K n=510^{-3}$, cross section of the mach at $\mathrm{x} / \mathrm{L}=0.3$

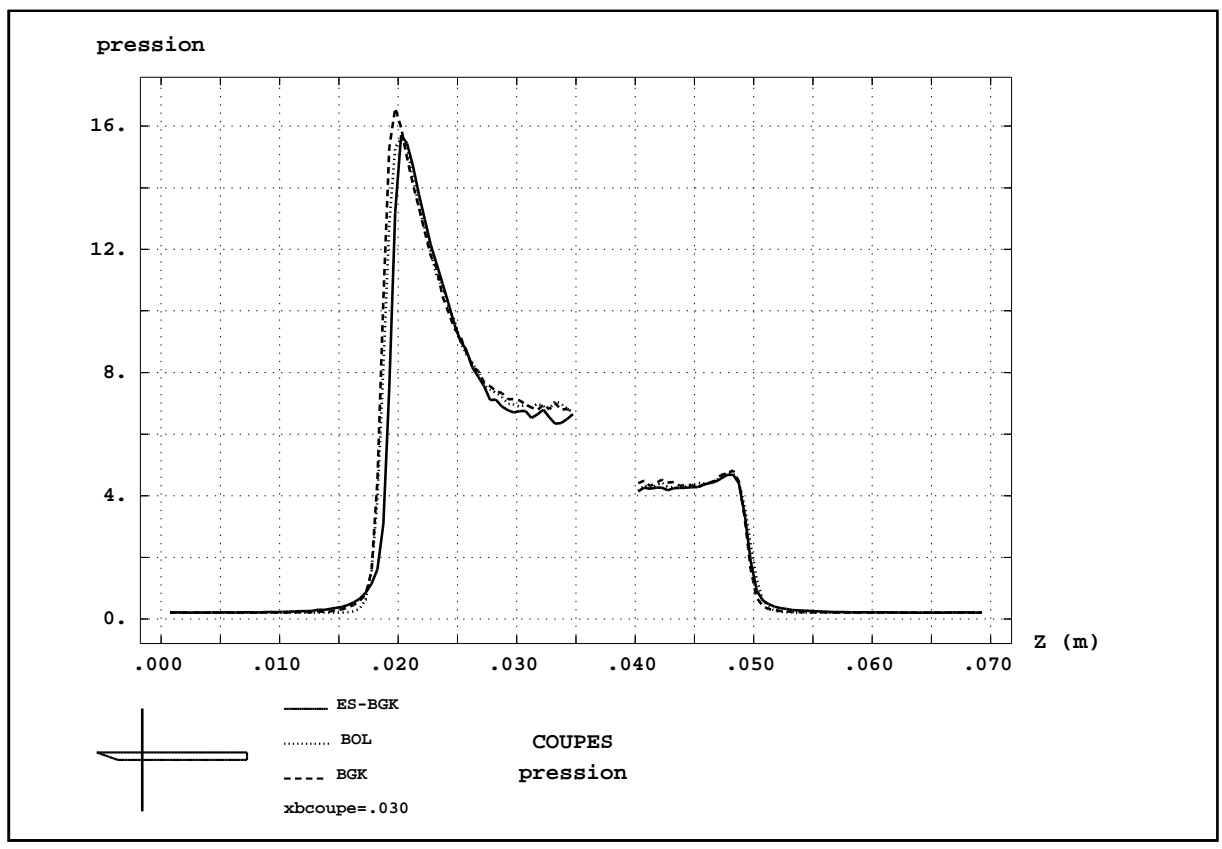

Figure 10: plate, $K n=510^{-3}$, cross section of the pressure at $\mathrm{x} / \mathrm{L}=0.3$ $\operatorname{RR} n^{\circ} 3872$ 


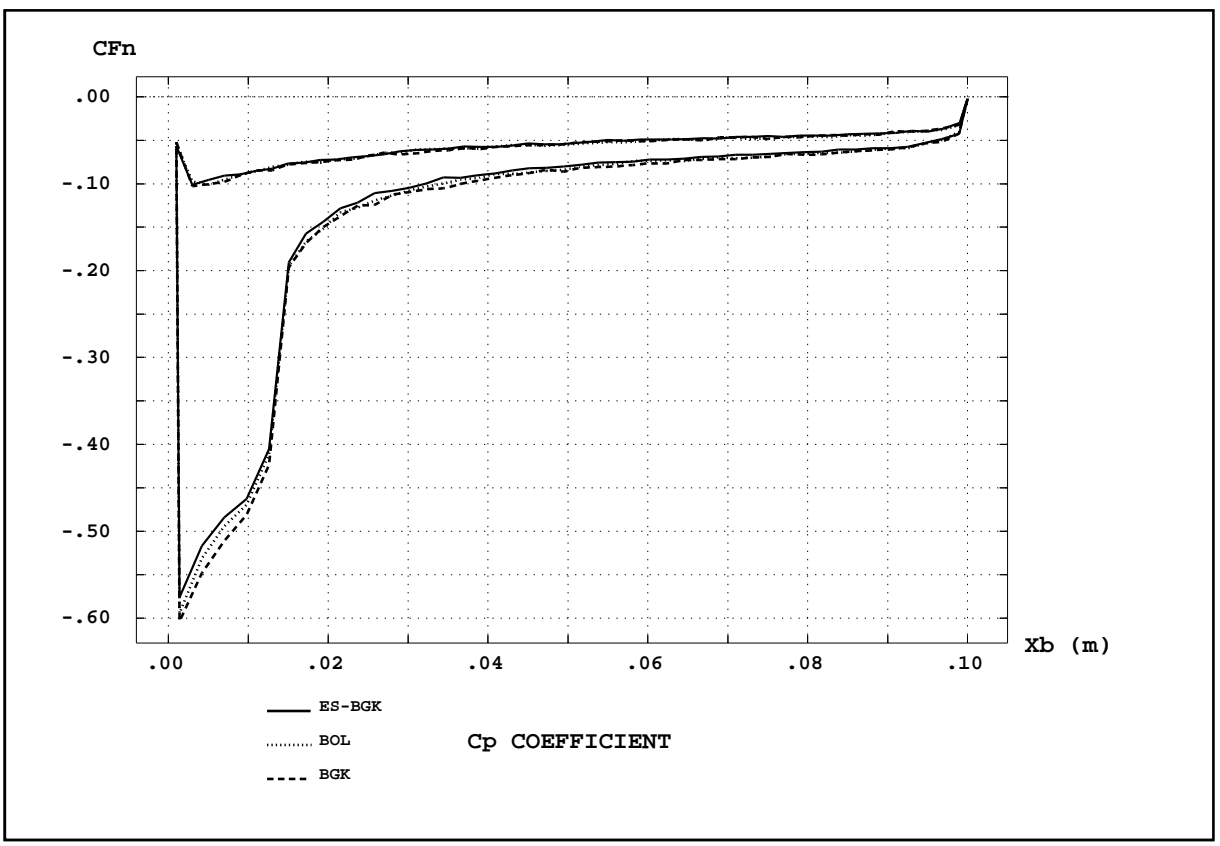

Figure 11: plate, $K n=510^{-3}$, Cp coefficient

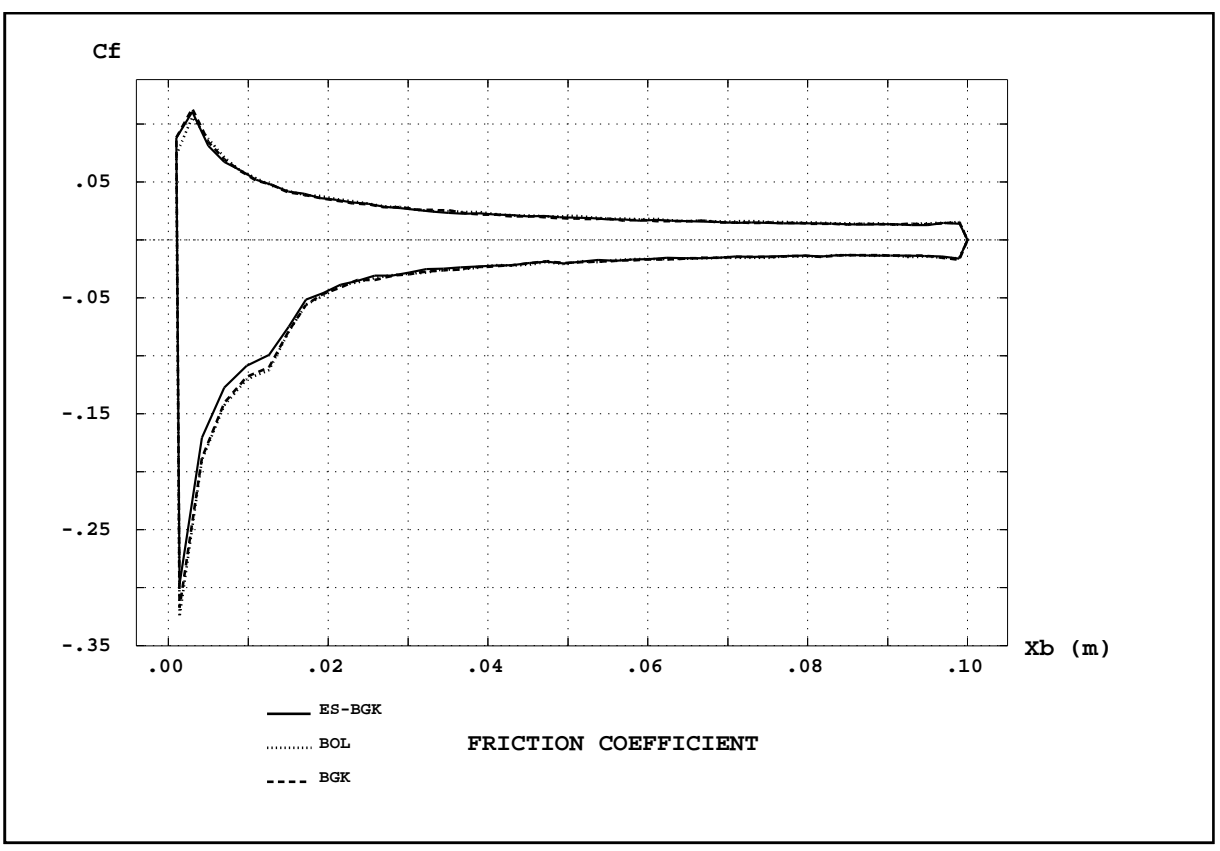

Figure 12: plate, $K n=510^{-3}$, Cf coefficient 


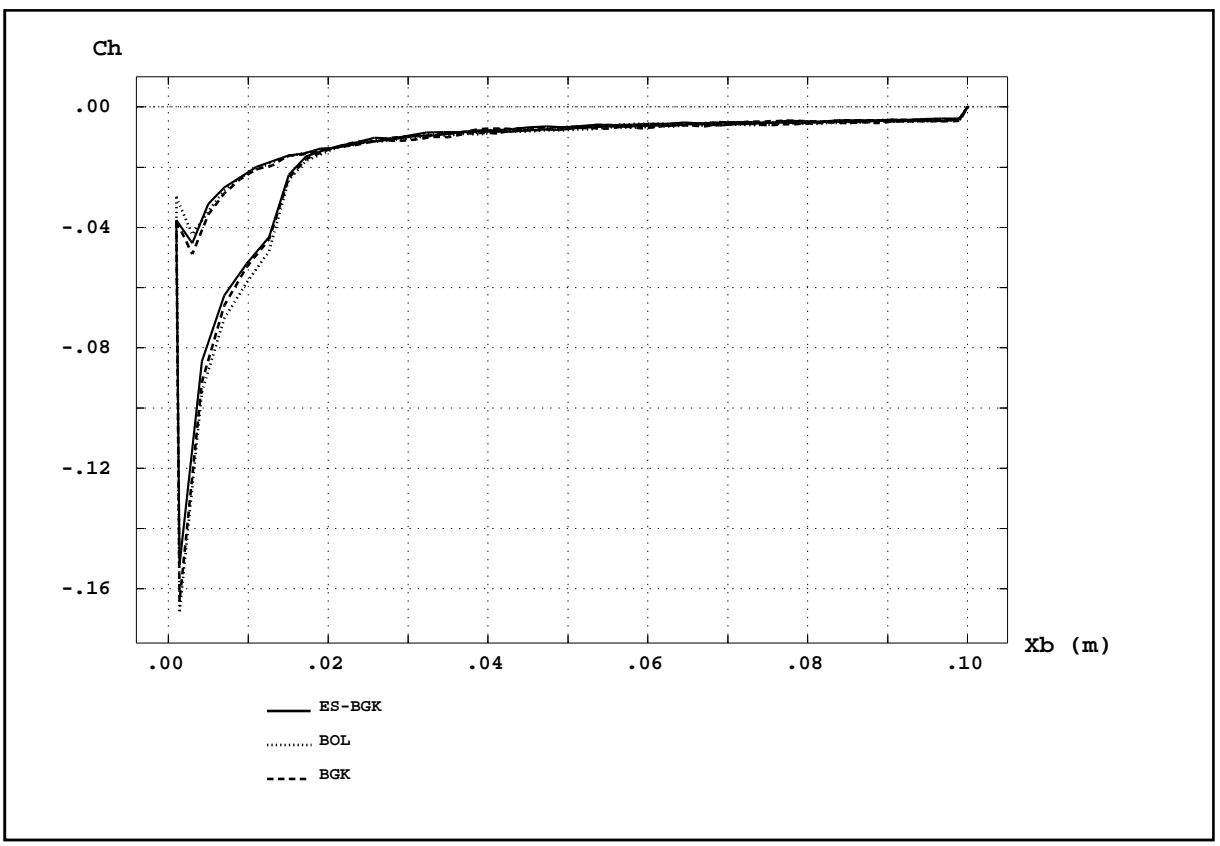

Figure 13: plate, $K n=510^{-3}$, Ch coefficient

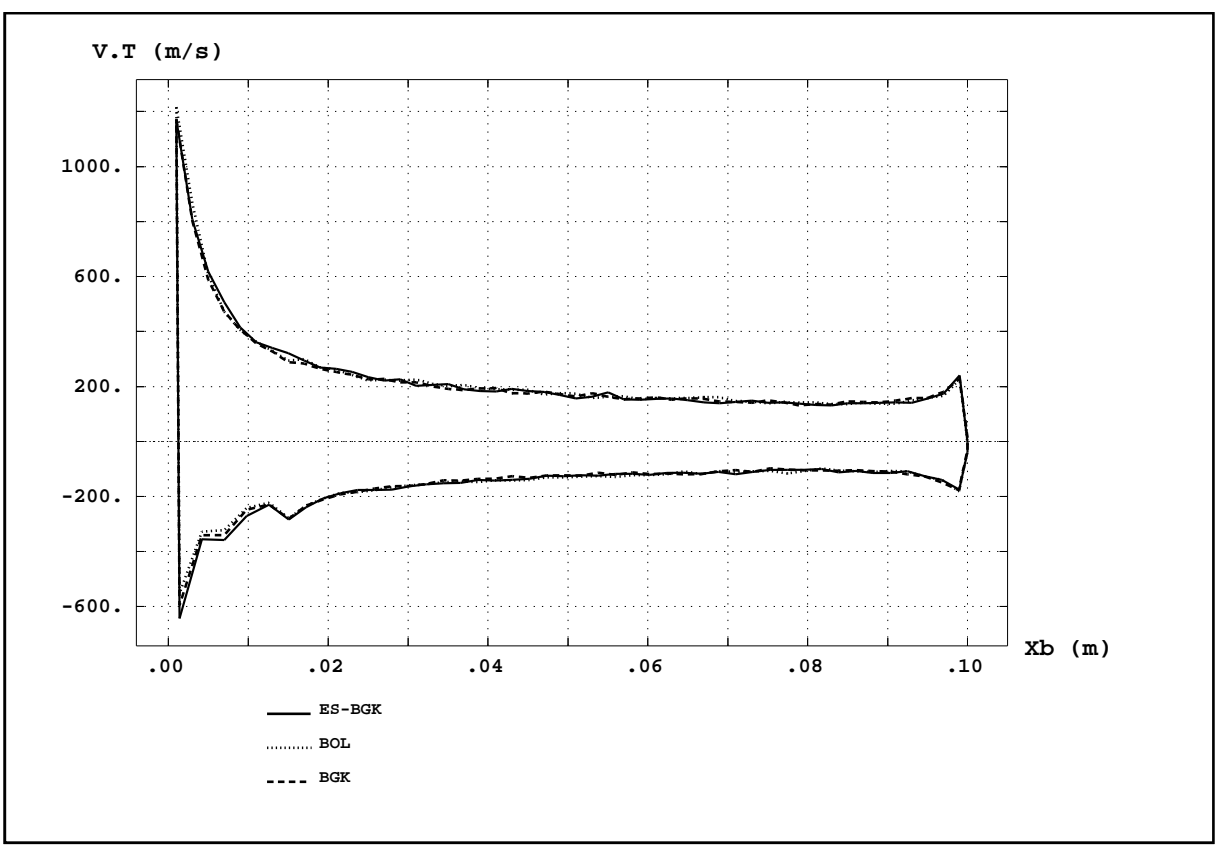

Figure 14: plate, $K n=510^{-3}$, tangential velocity

$\mathrm{RR} \mathrm{n}^{\circ} 3872$ 


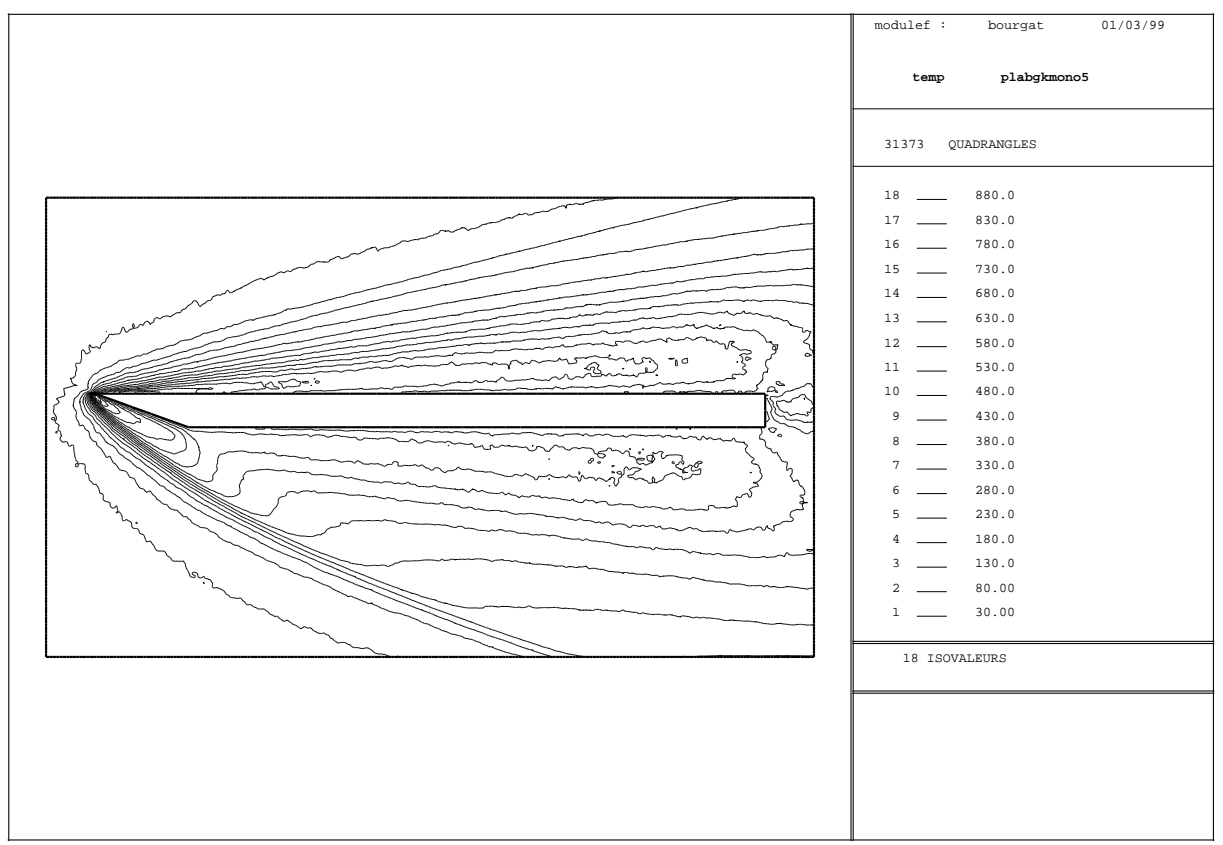

Figure 15: plate, $K n=1.4410^{-2}$, ES-BGK isotemperatures

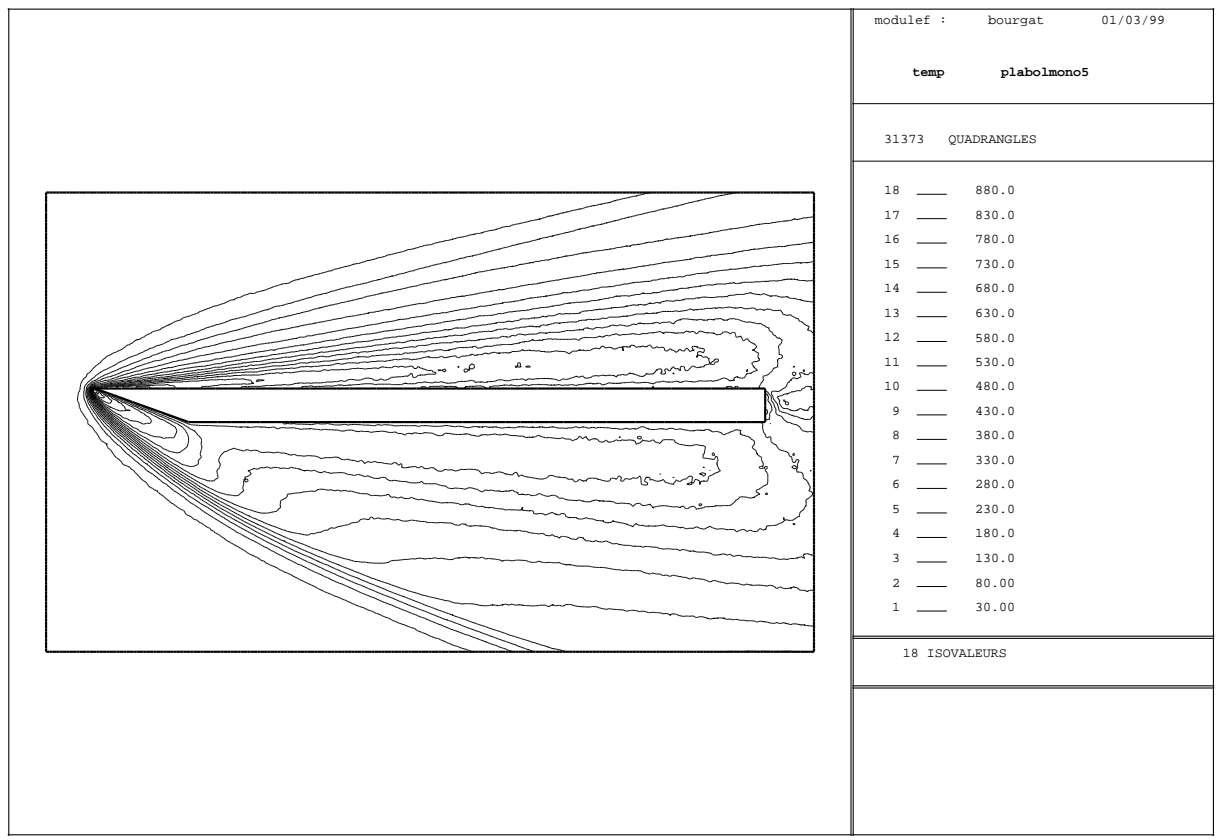

Figure 16: plate, $K n=1.4410^{-2}$, Boltzmann isotemperatures 


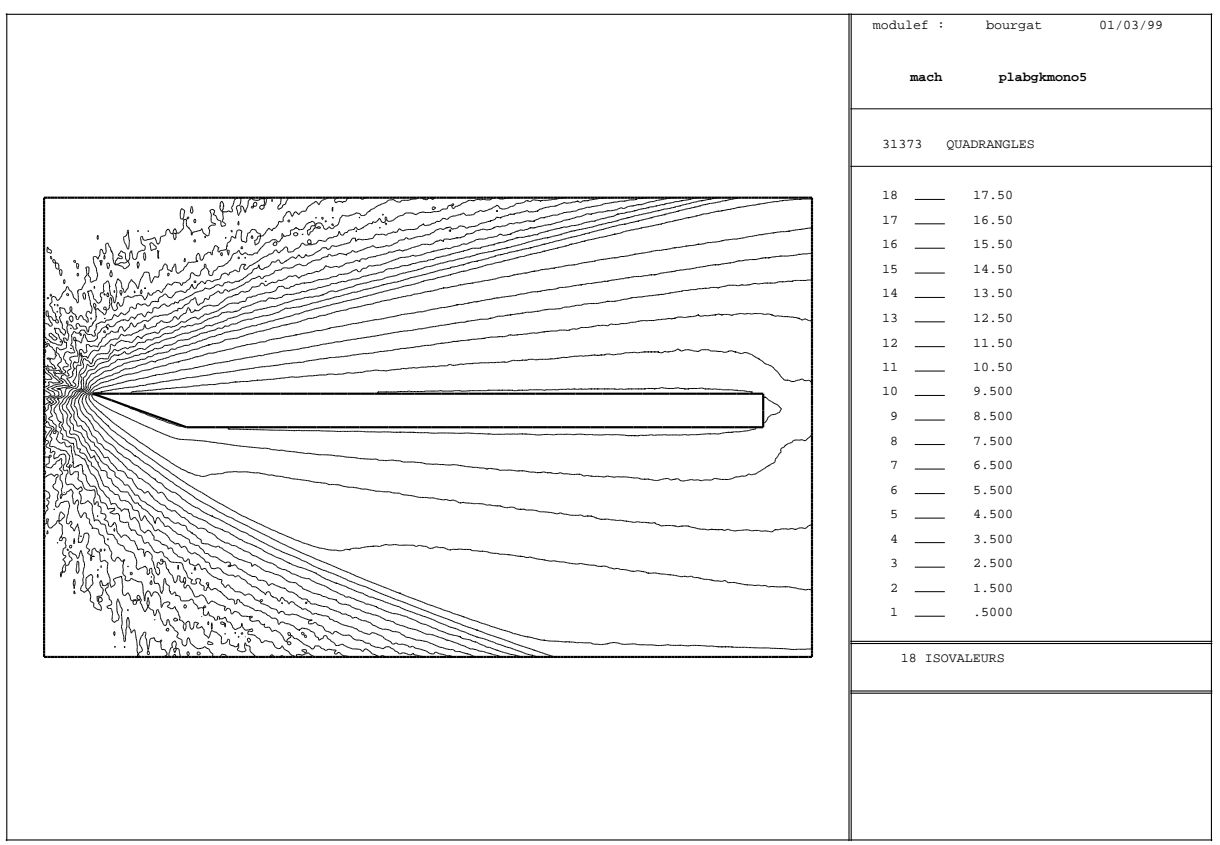

Figure 17: plate, $K n=1.4410^{-2}$, ES-BGK isomachs

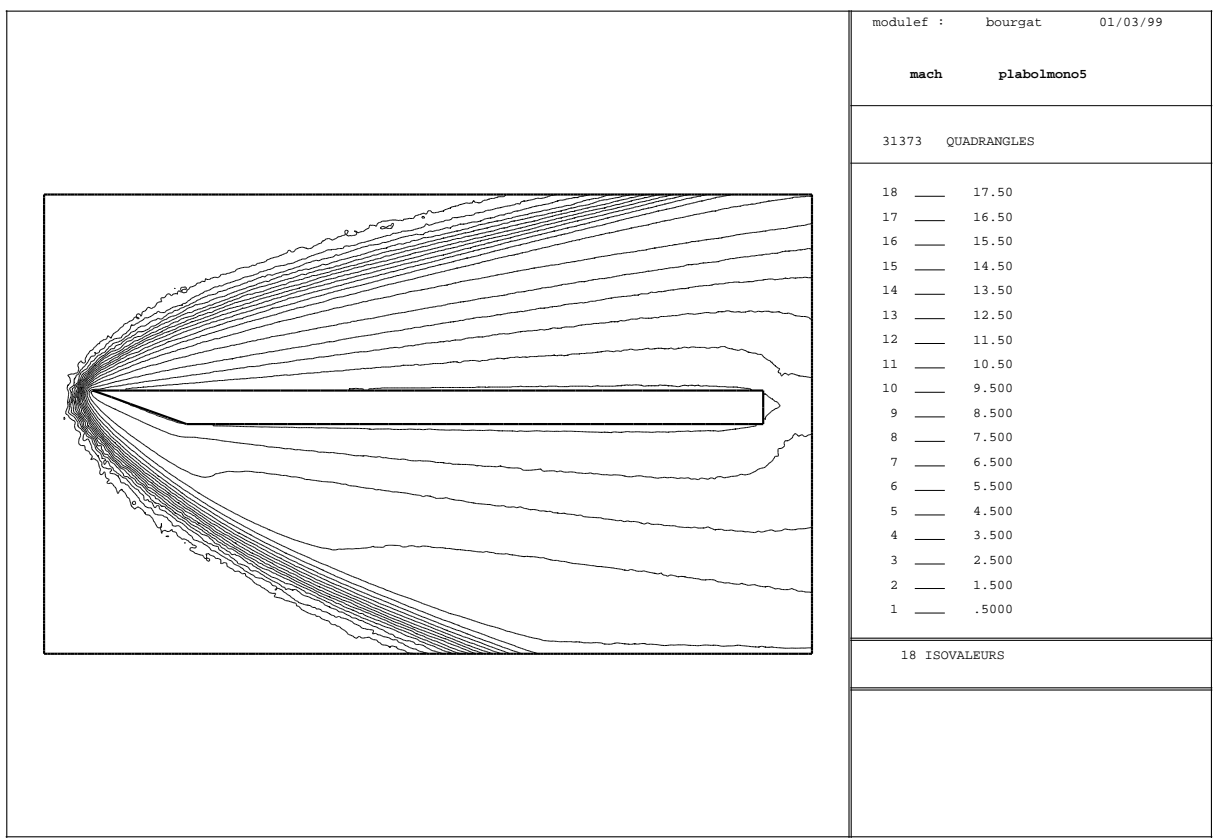

Figure 18: plate, $K n=1.4410^{-2}$, Boltzmann isomachs

$\operatorname{RR} n^{\circ} 3872$ 


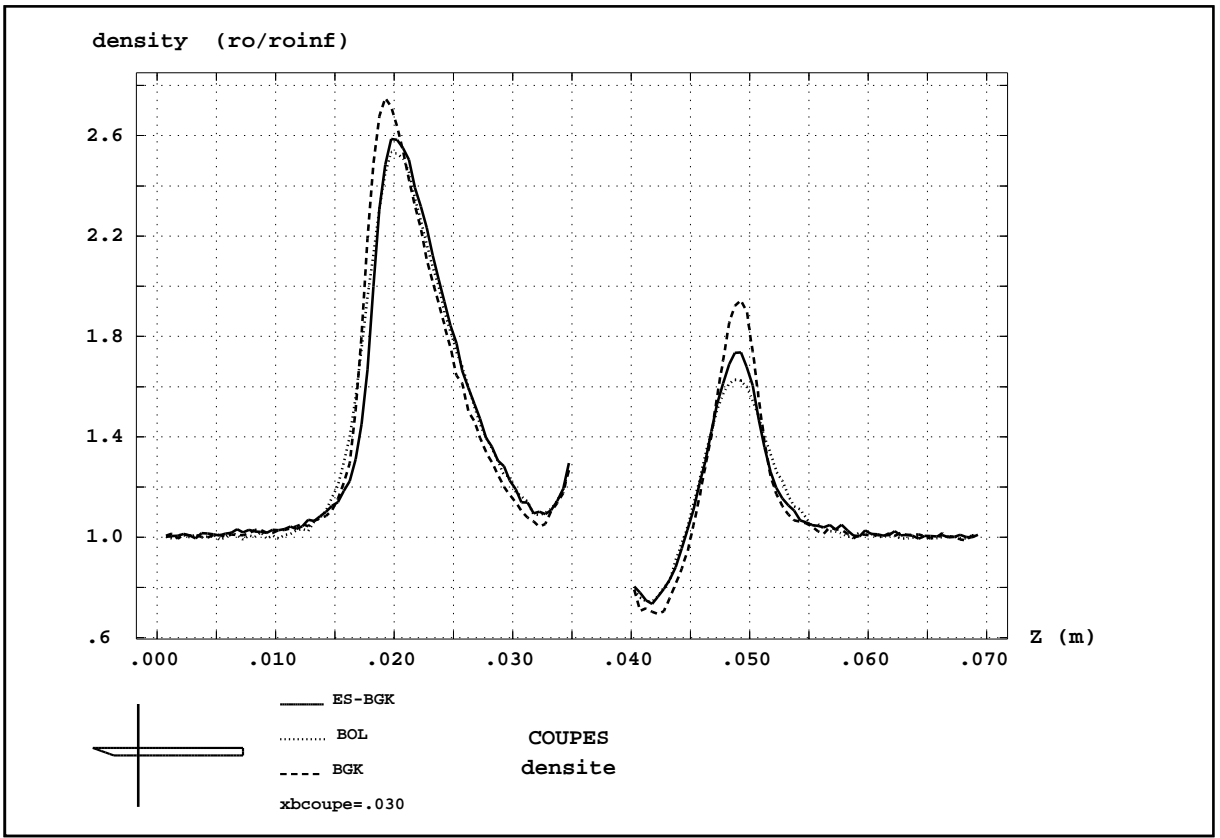

Figure 19: plate, $K n=1.4410^{-2}$, crosss section of the density at $\mathrm{x} / \mathrm{L}=0.3$

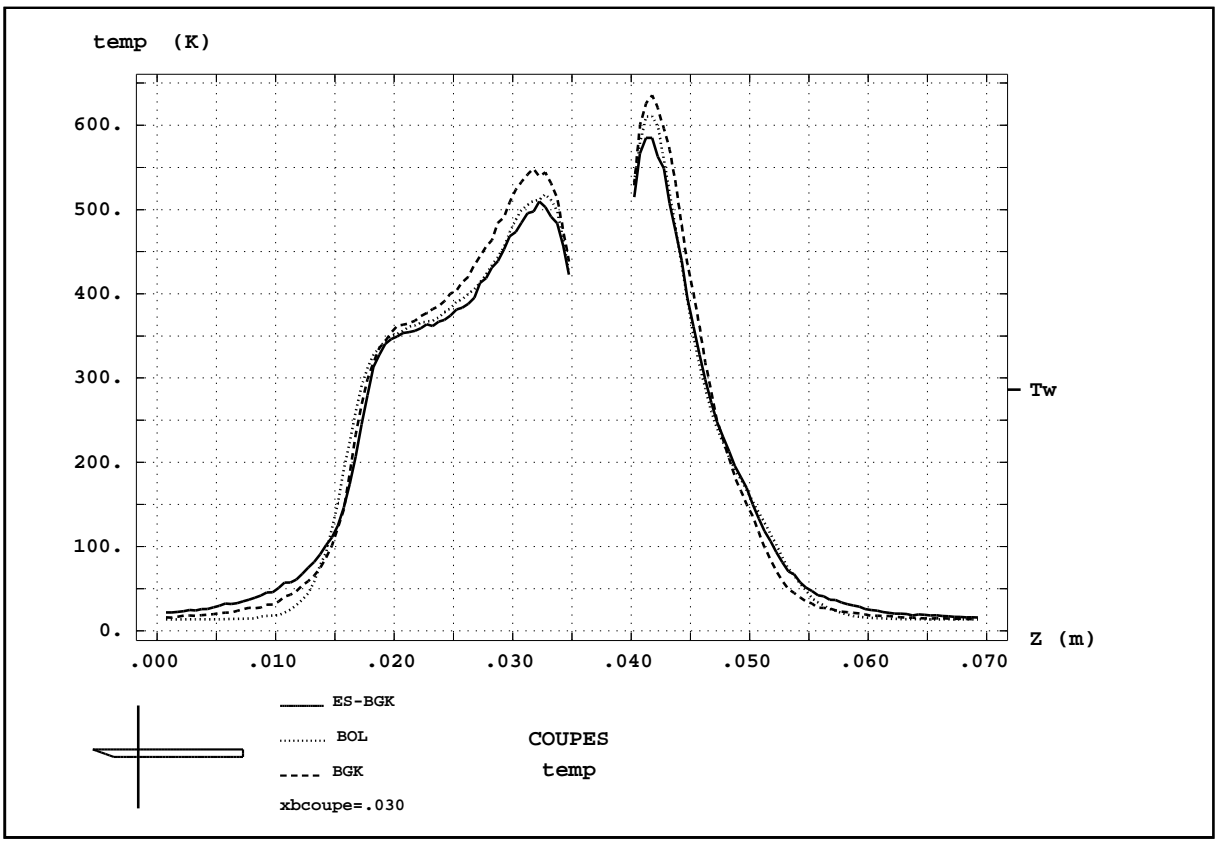

Figure 20: plate, $K n=1.4410^{-2}$, cross section of the temperature at $\mathrm{x} / \mathrm{L}=0.3$ 


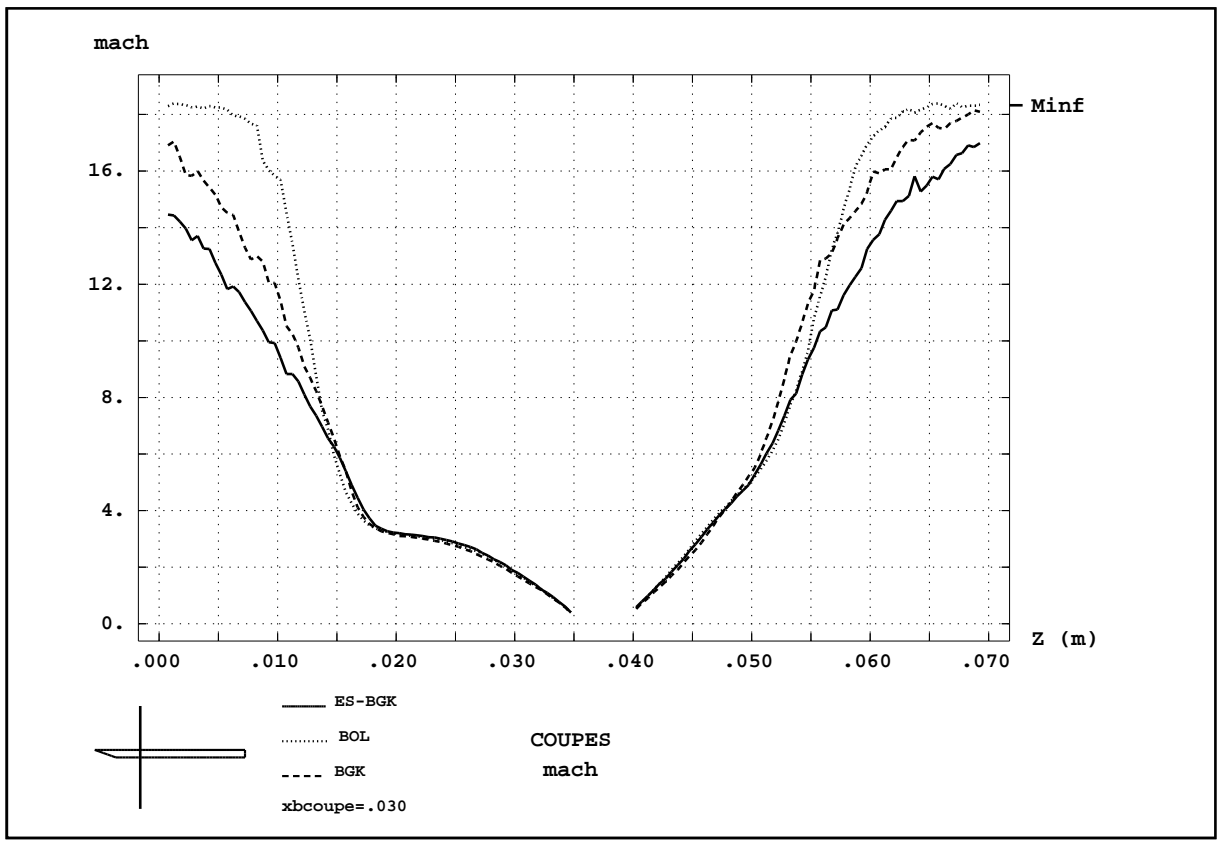

Figure 21: plate, $K n=1.4410^{-2}$, cross section of the mach at $\mathrm{x} / \mathrm{L}=0.3$

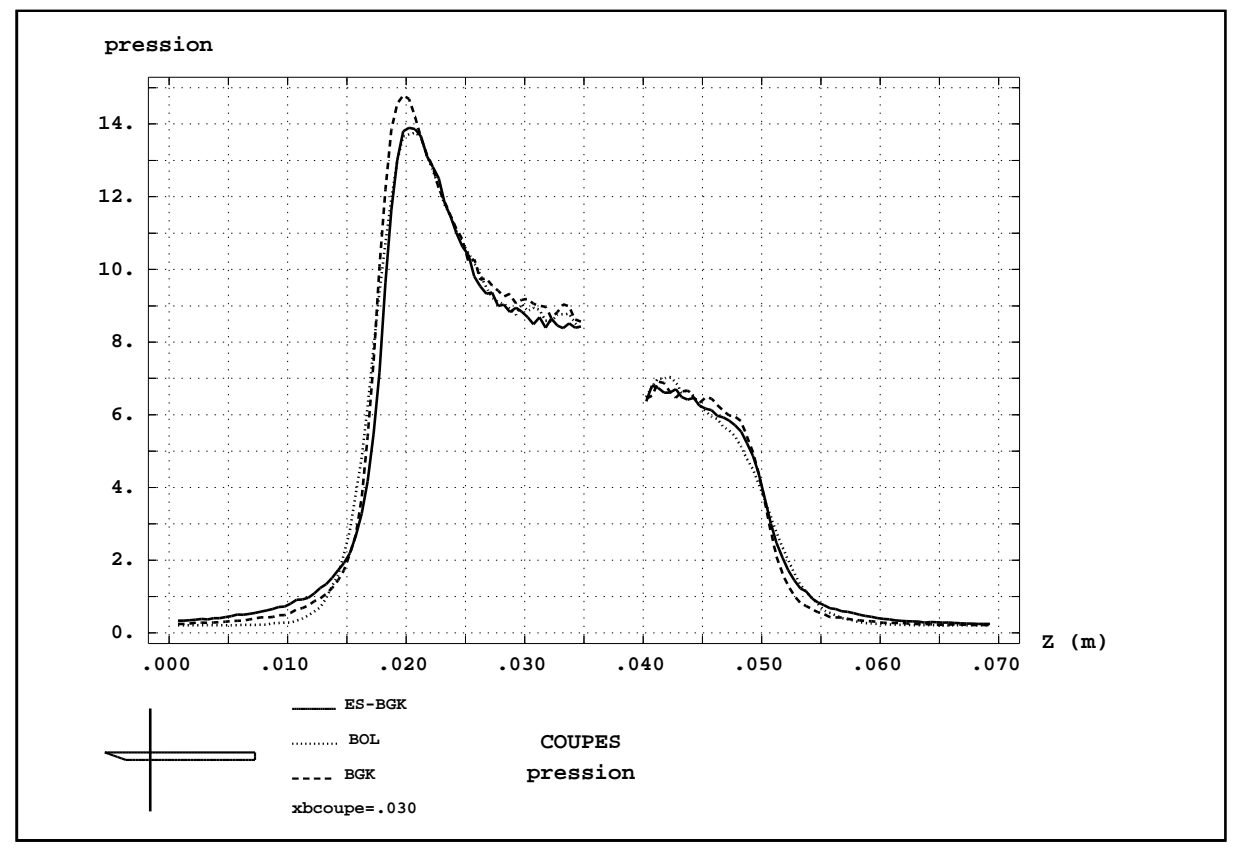

Figure 22: plate, $K n=1.4410^{-2}$, cross section of the pressure at $\mathrm{x} / \mathrm{L}=0.3$ $\operatorname{RR} n^{\circ} 3872$ 


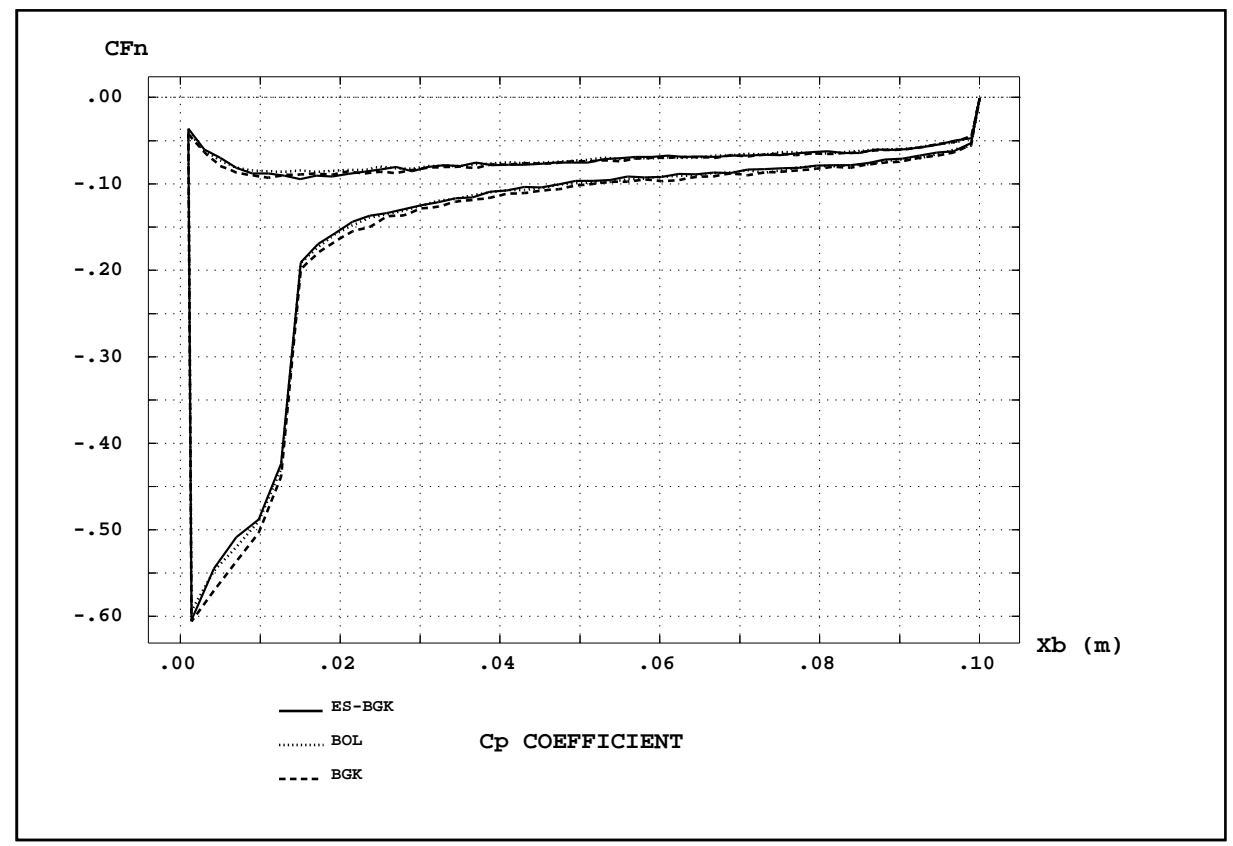

Figure 23: plate, $K n=1.4410^{-2}$, Cp coefficient

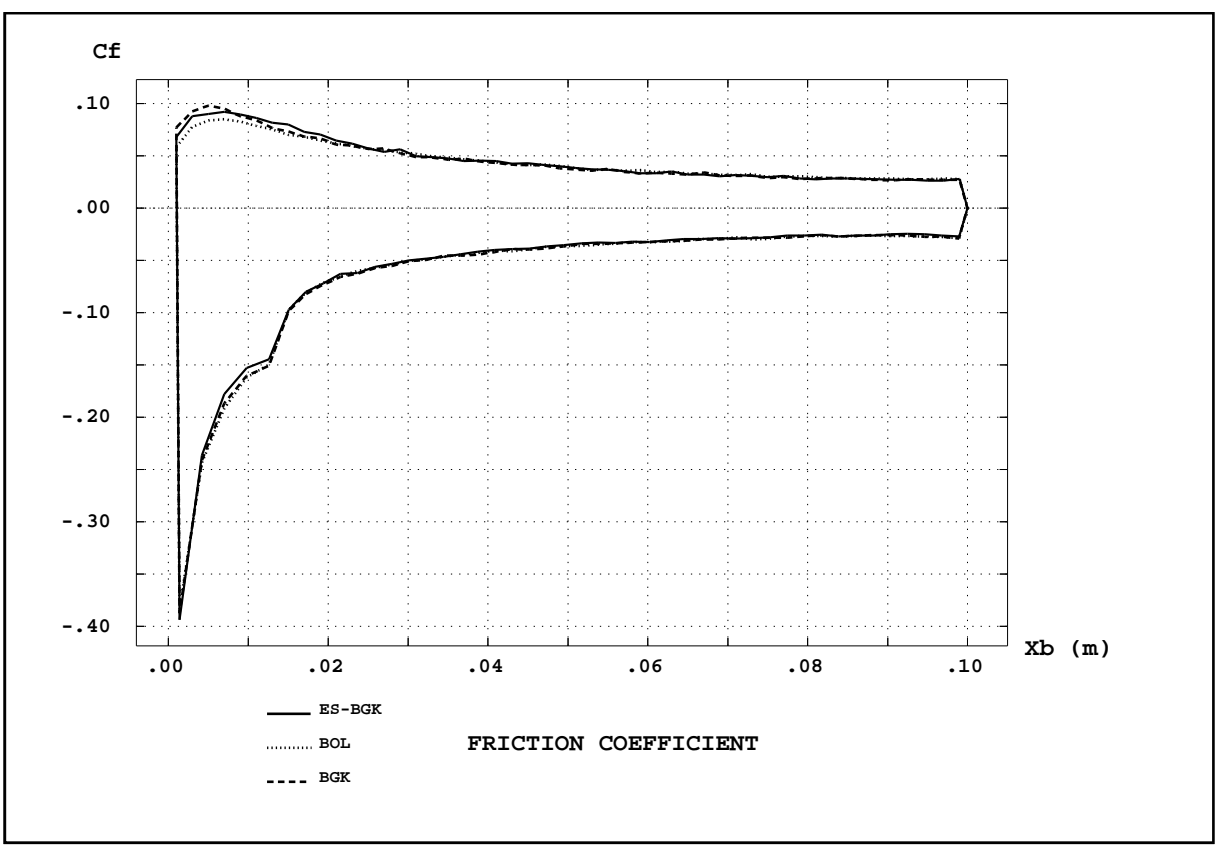

Figure 24: plate, $K n=1.4410^{-2}$, Cf coefficient 


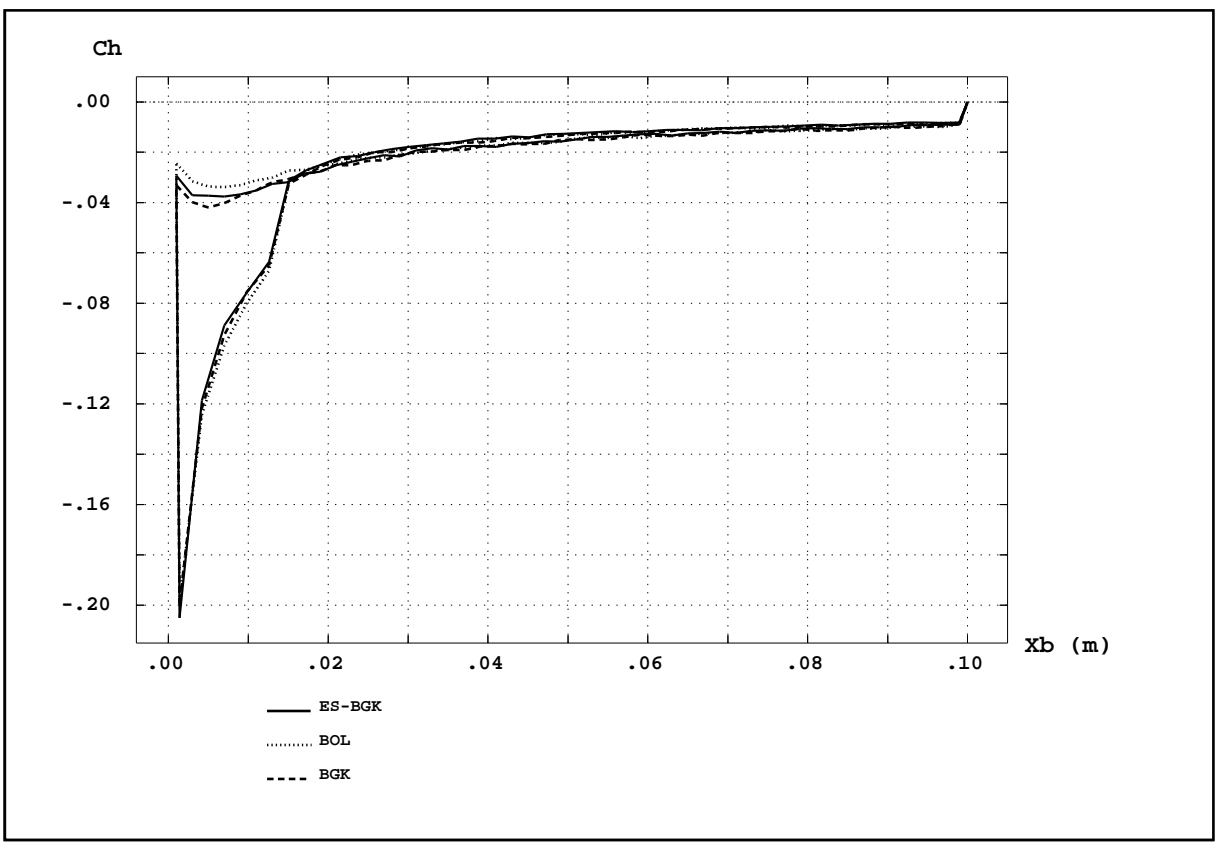

Figure 25: plate, $K n=1.4410^{-2}$, Ch coefficient

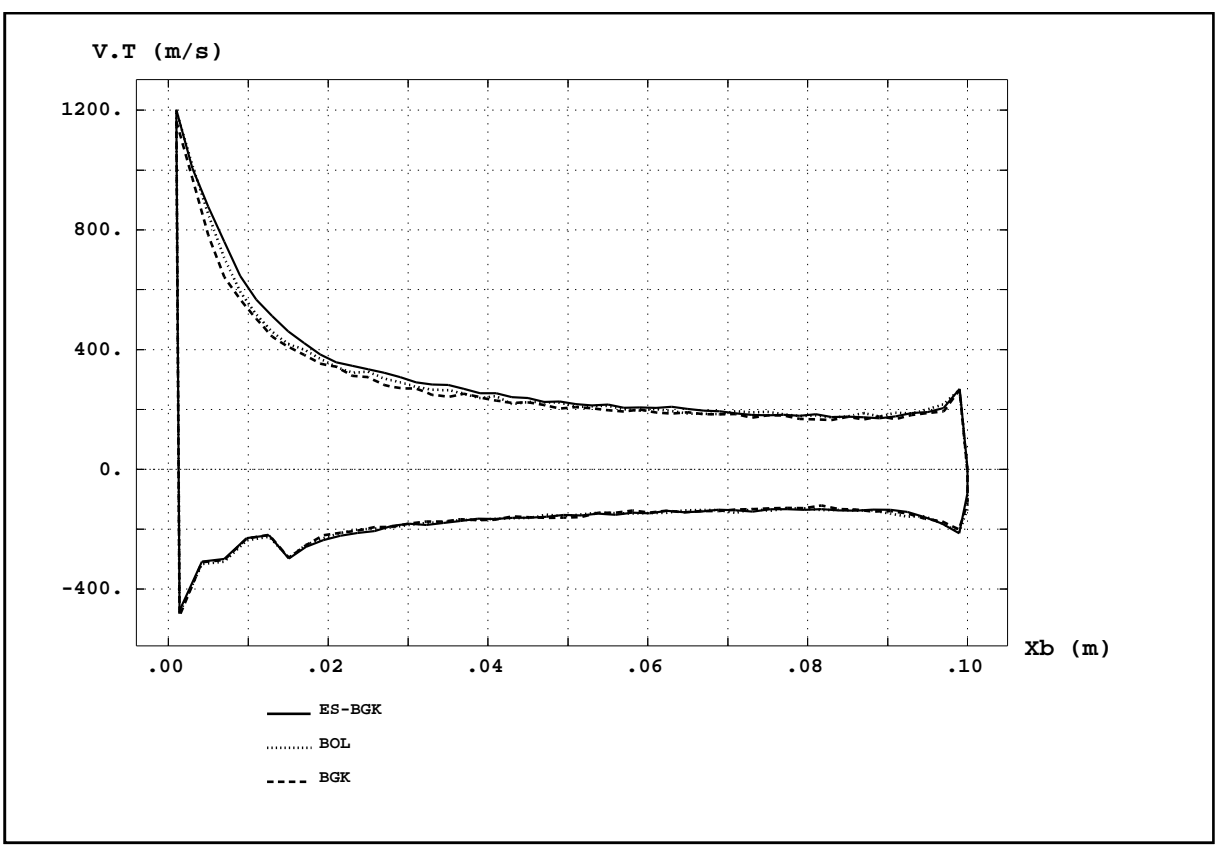

Figure 26: plate, $K n=1.4410^{-2}$, tangential velocity

$\mathrm{RR} \mathrm{n}^{\circ} 3872$ 


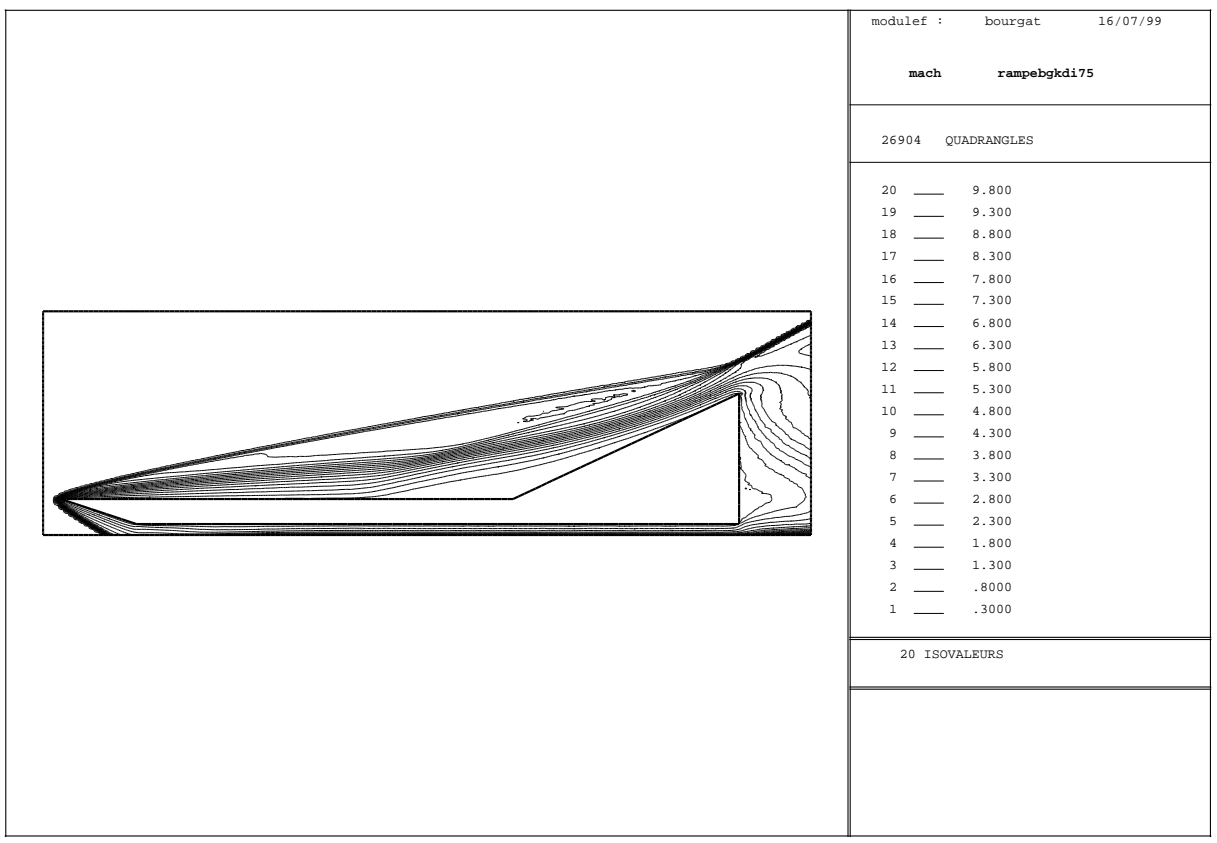

Figure 27: ramp, diatomic, ES-BGK isomachs

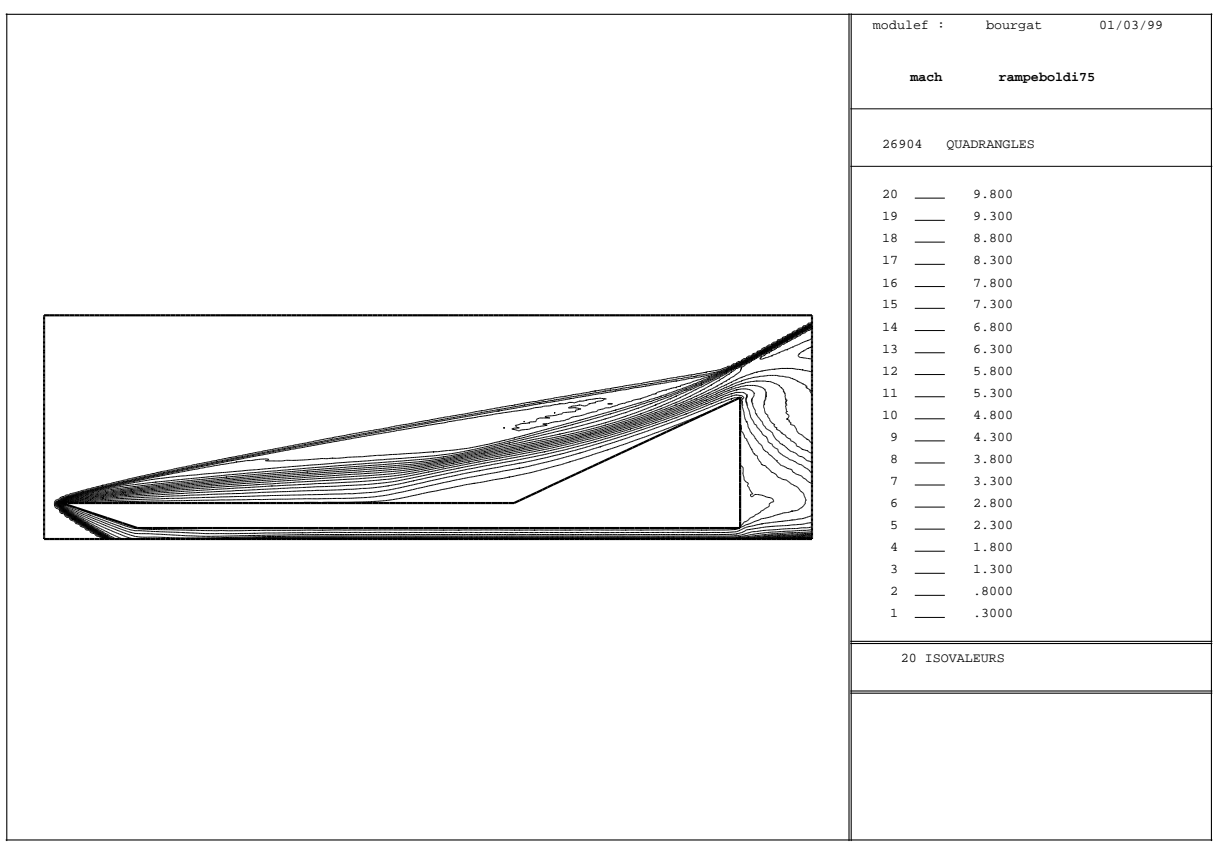

Figure 28: ramp, diatomic, Boltzmann isomachs 


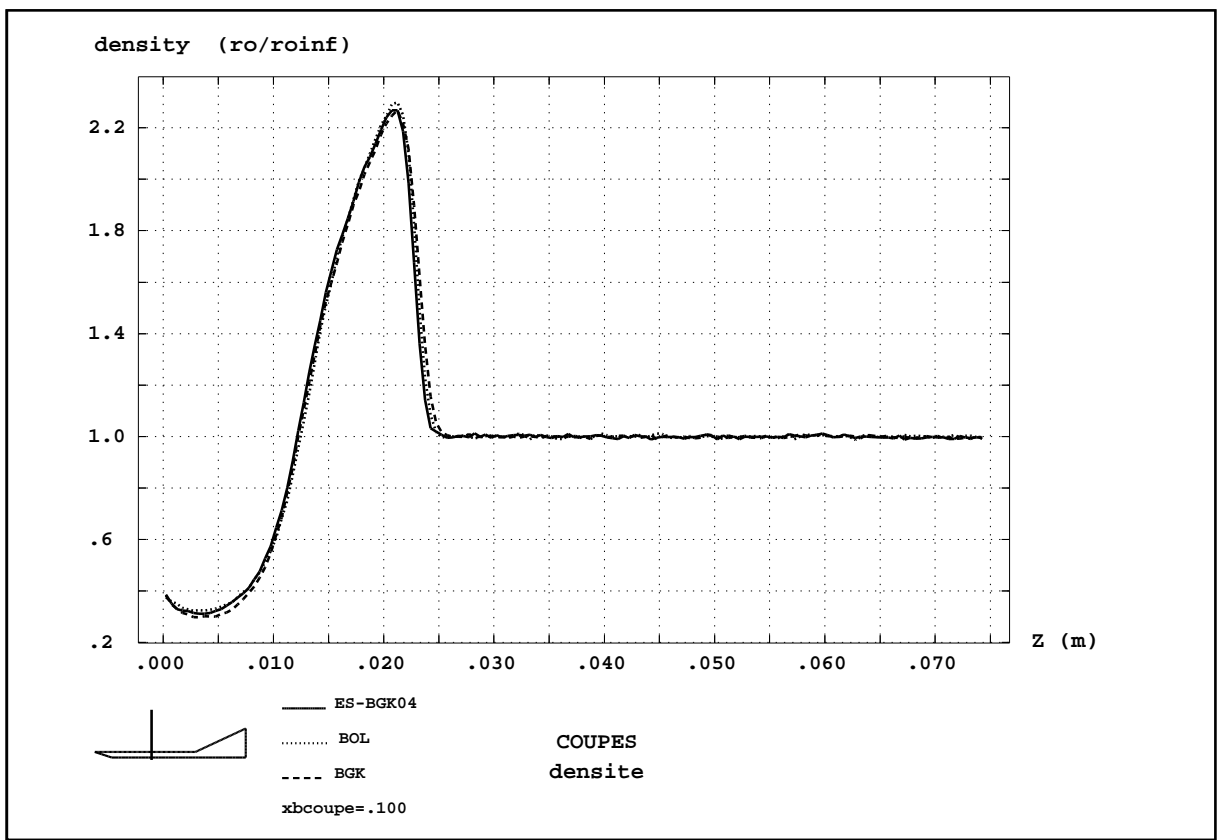

Figure 29: ramp, diatomic, cross section of the density at $\mathrm{x}=0.1$

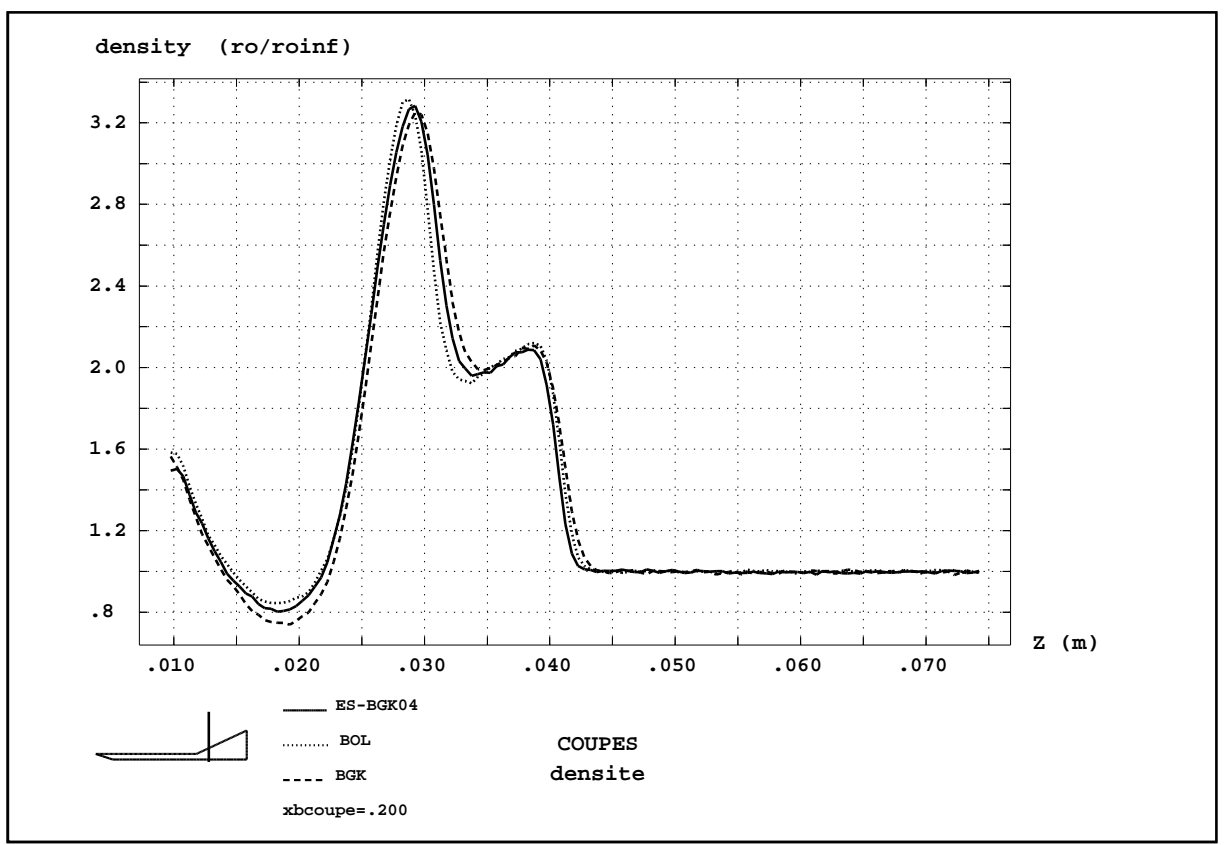

Figure 30: ramp, diatomic, cross section of the density at $\mathrm{x}=0.2$

$\mathrm{RR} \mathrm{n}^{\circ} 3872$ 


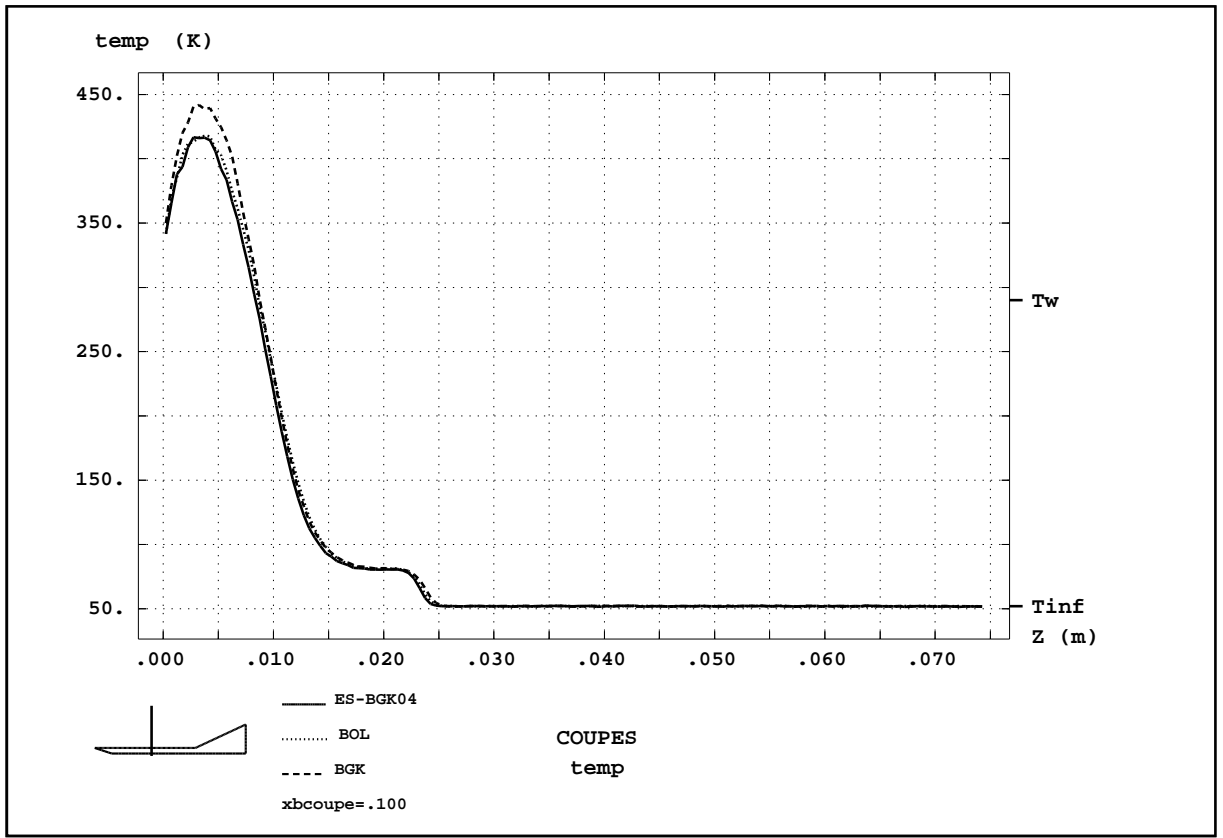

Figure 31: ramp, diatomic, cross section of the temperature at $\mathrm{x}=0.1$

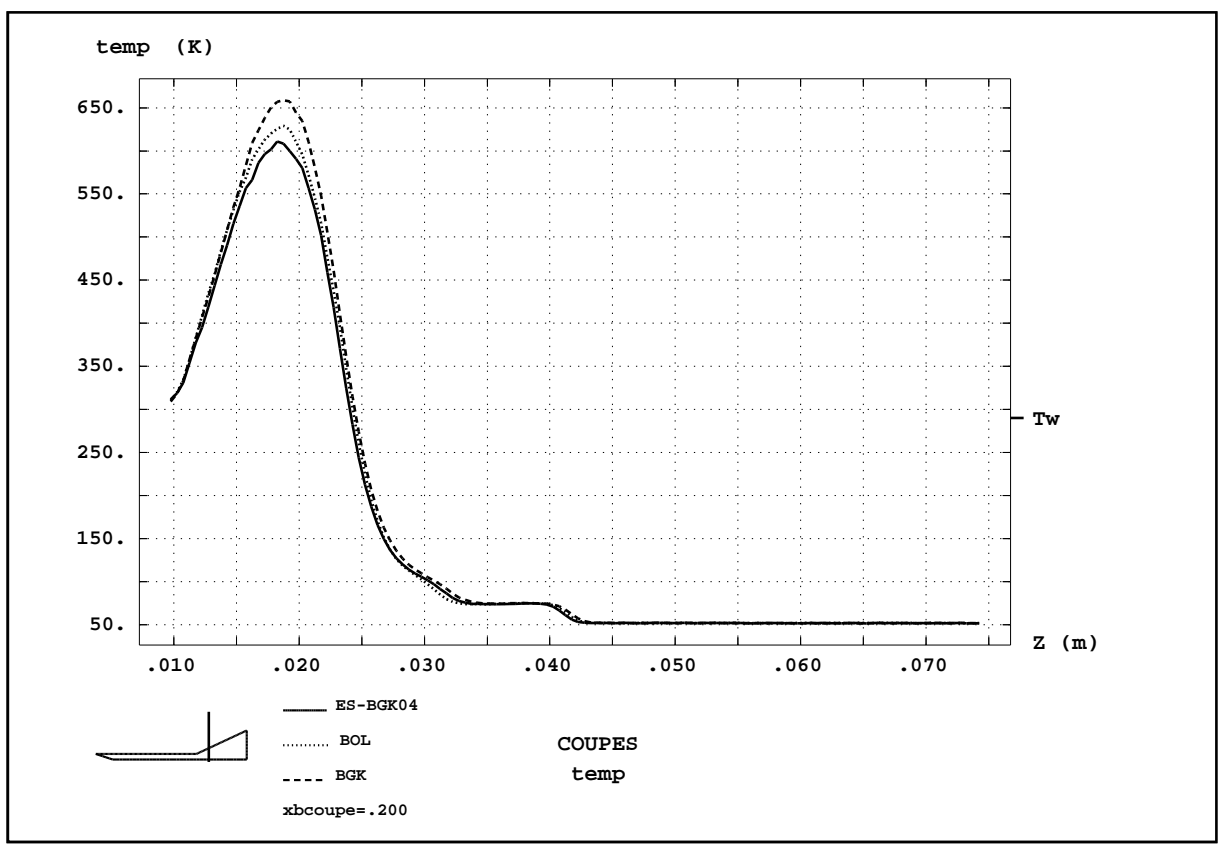

Figure 32: ramp, diatomic, cross section of the temperature at $\mathrm{x}=0.2$ 


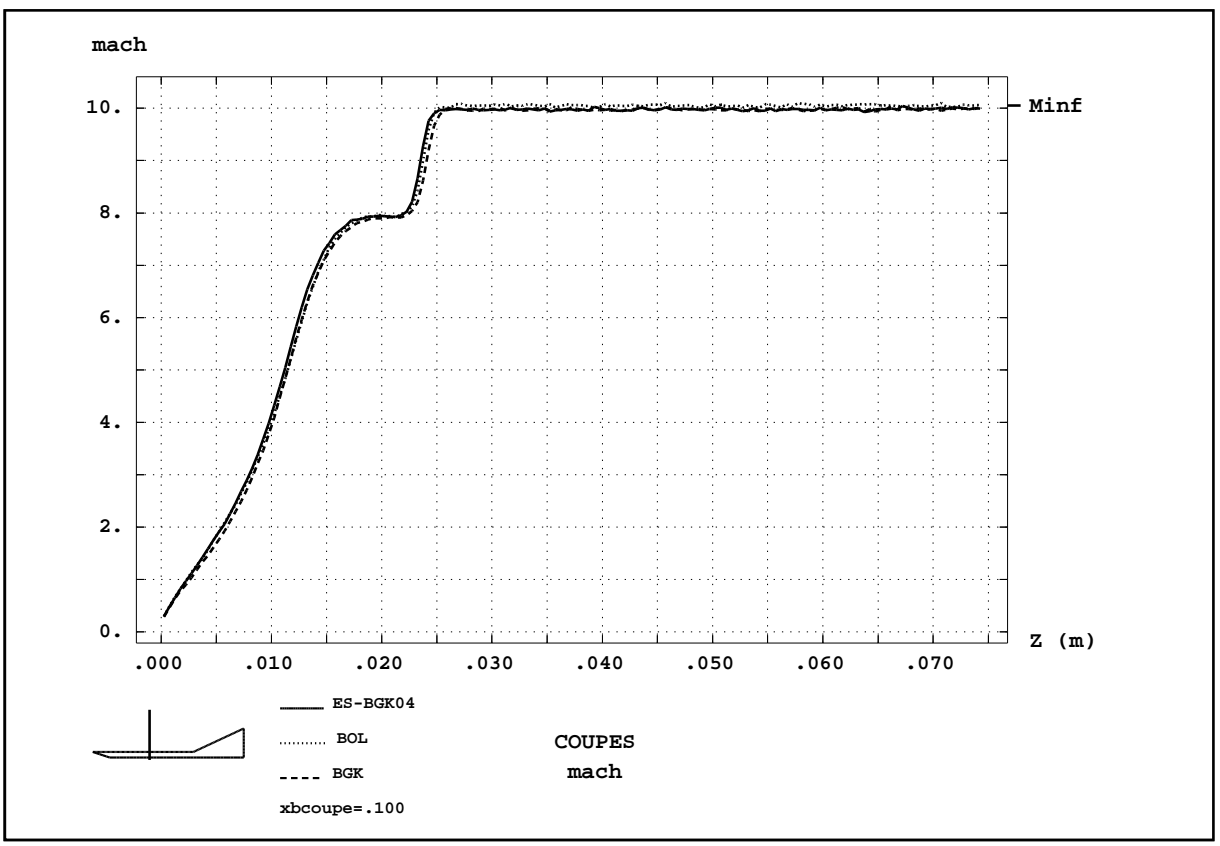

Figure 33: ramp, diatomic, cross section of the mach at $\mathrm{x}=0.1$

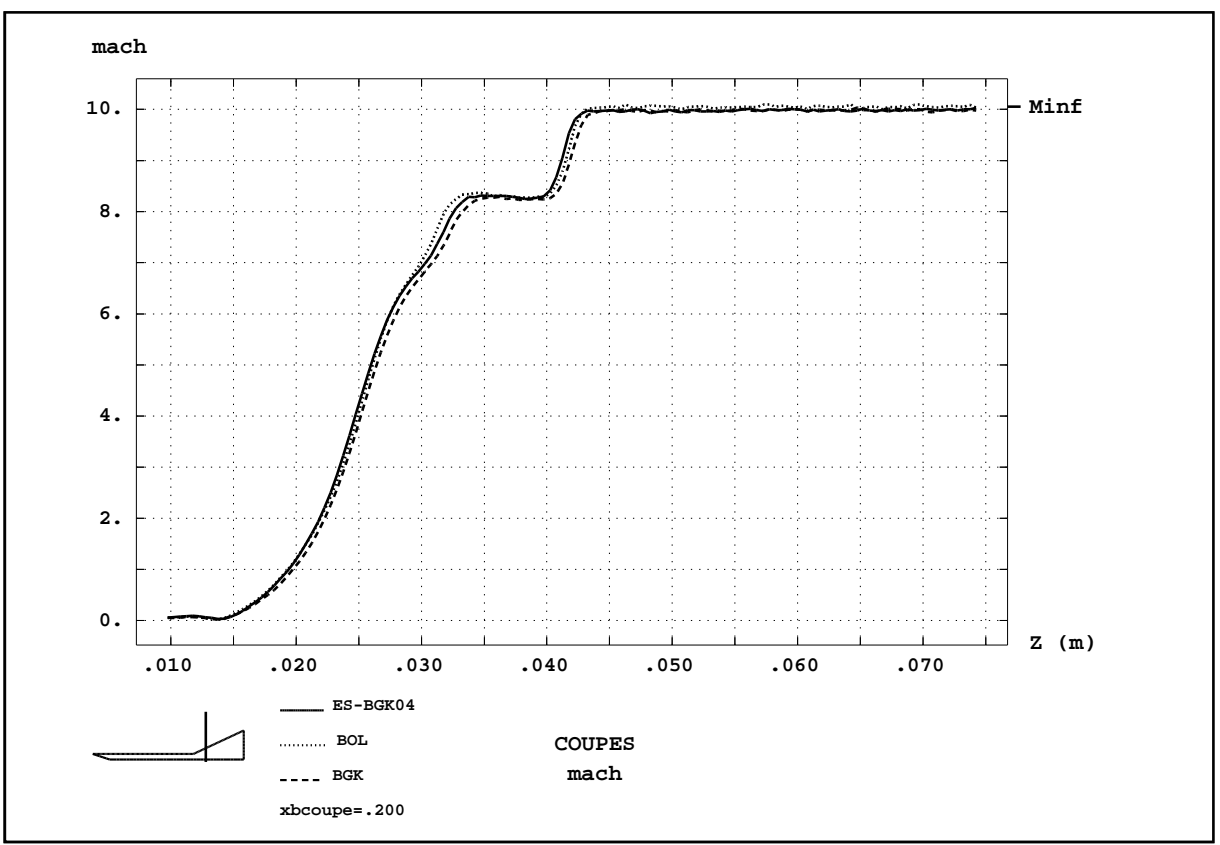

Figure 34: ramp, diatomic, cross section of the mach at $\mathrm{x}=0.2$

$\mathrm{RR} \mathrm{n}^{\circ} 3872$ 


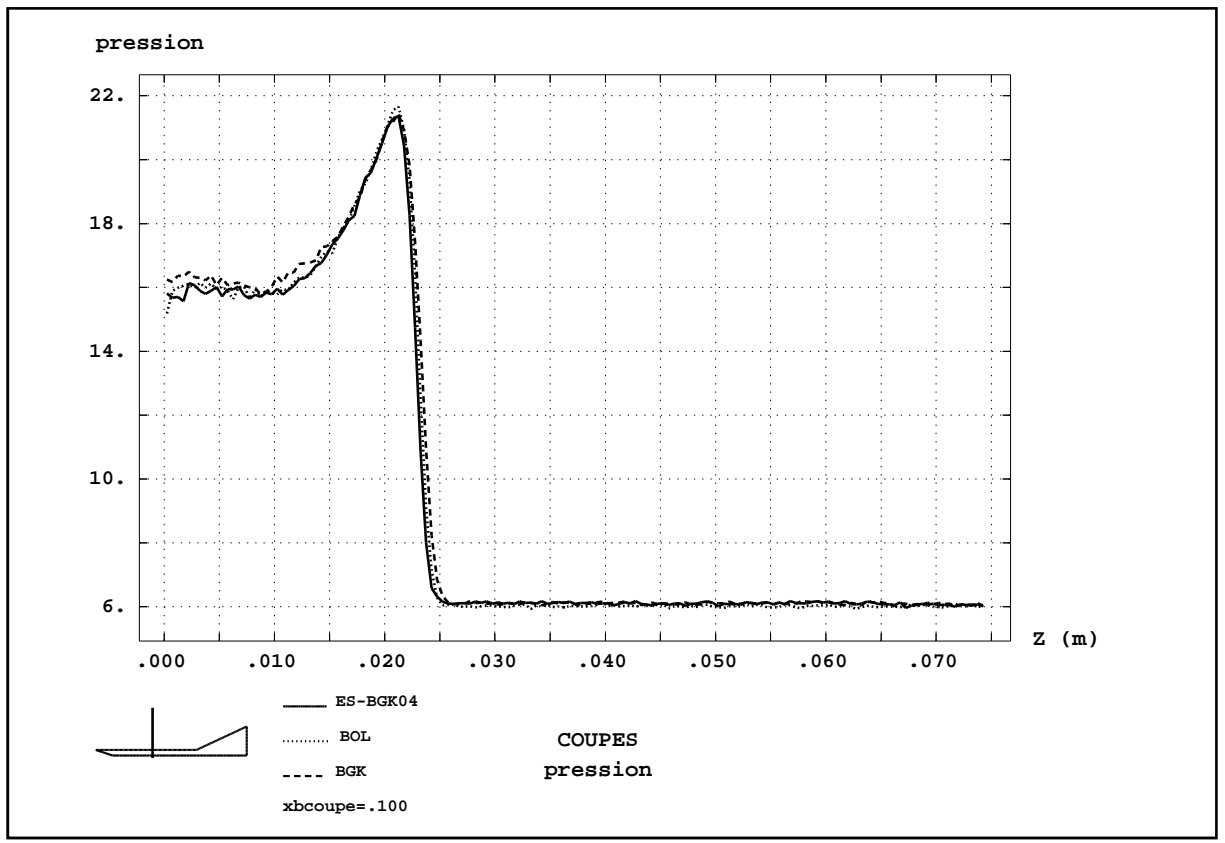

Figure 35: ramp, diatomic, cross section of the pressure at $\mathrm{x}=0.1$

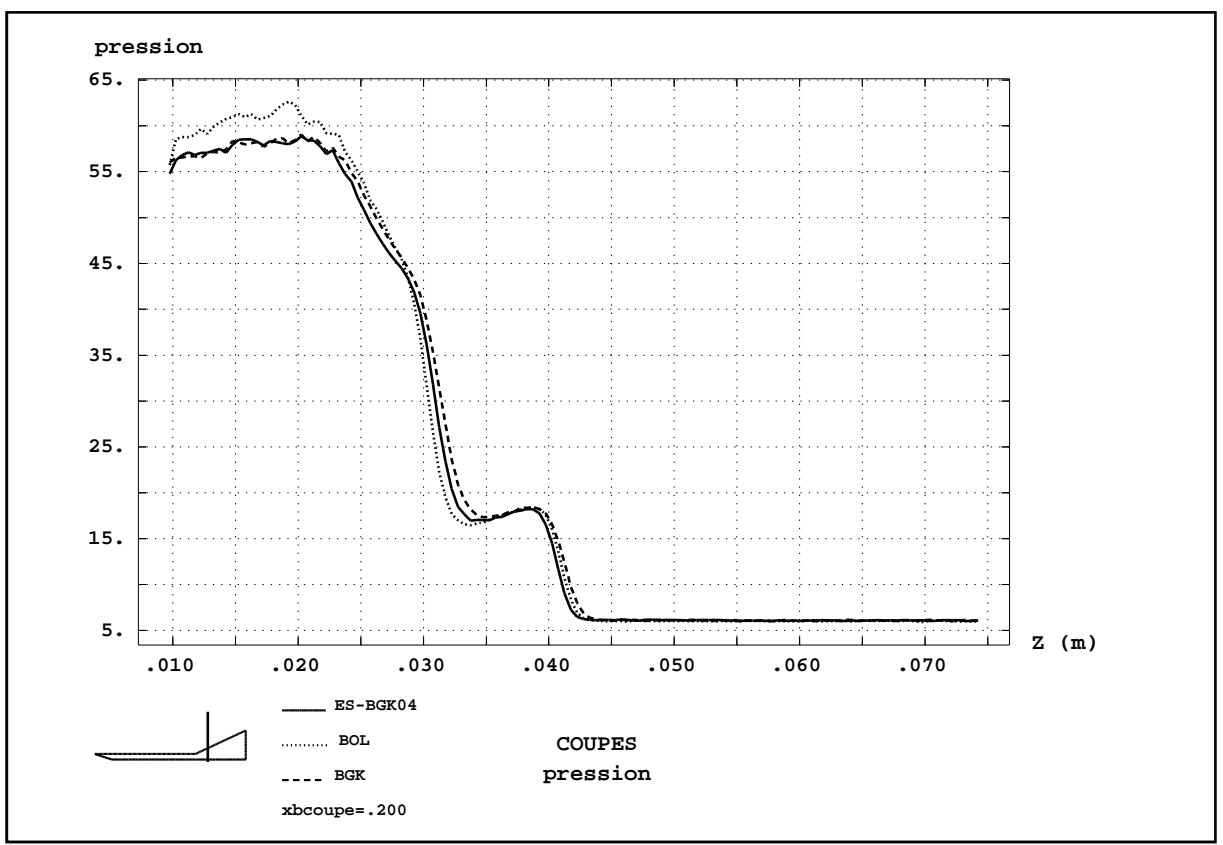

Figure 36: ramp, diatomic, cross section of the pressure at $\mathrm{x}=0.2$ 


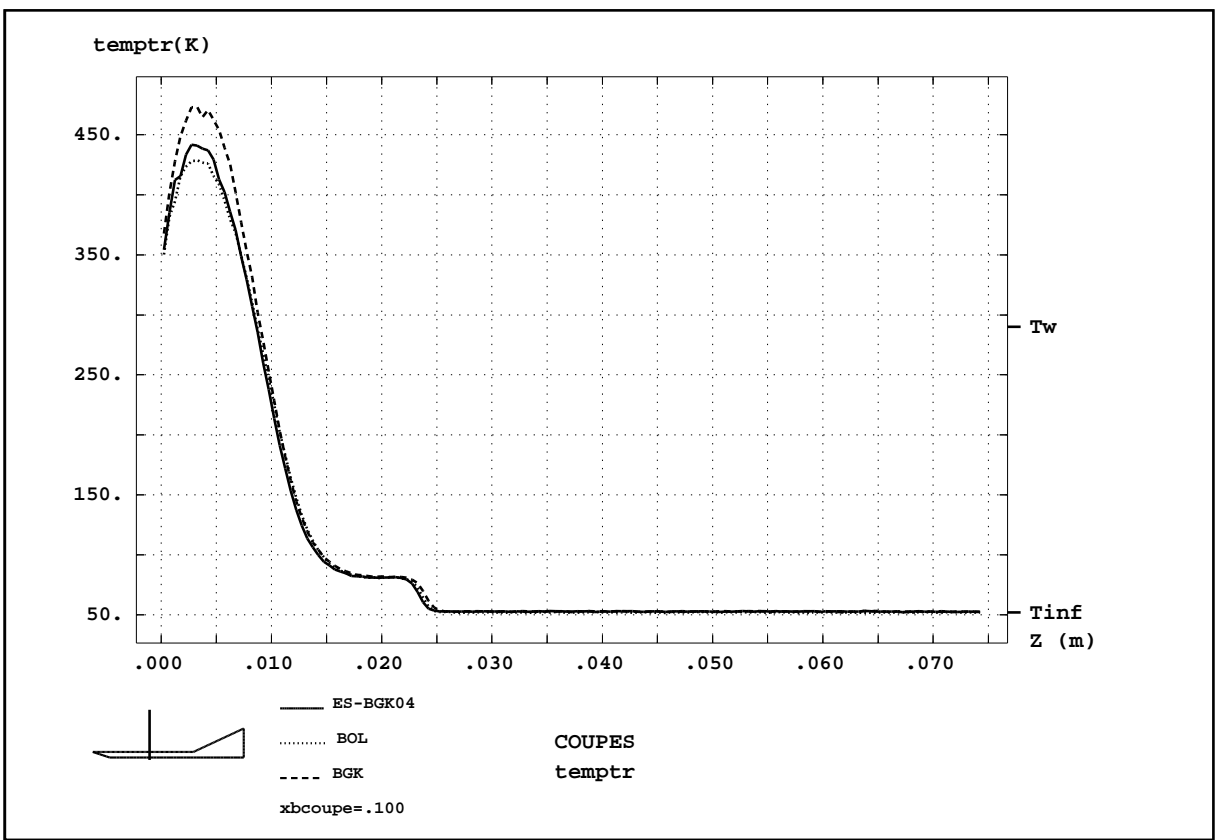

Figure 37: ramp, diatomic, cross section of the translational temperature at $\mathrm{x}=0.1$

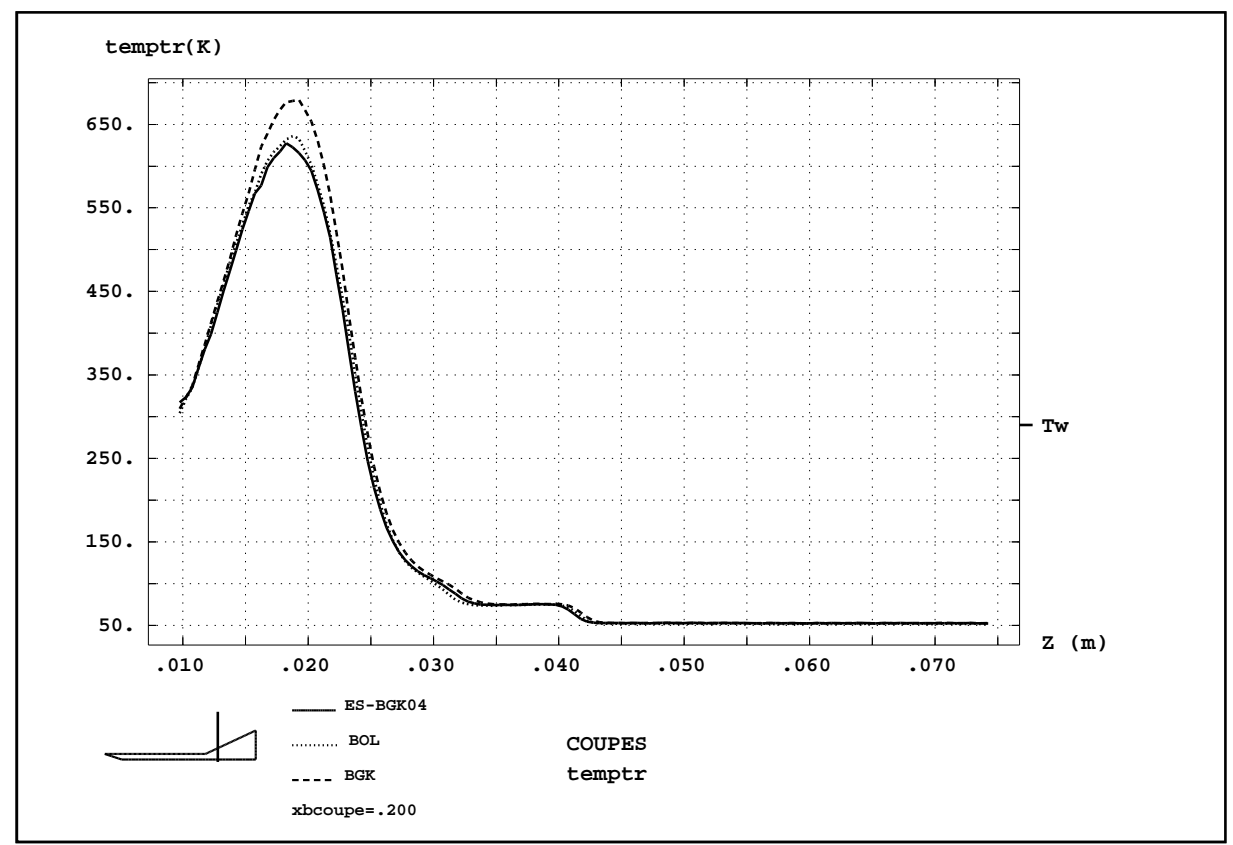

Figure 38: ramp, diatomic, cross section of the translational temperature at $\mathrm{x}=0.2$ $\operatorname{RR} n^{\circ} 3872$ 


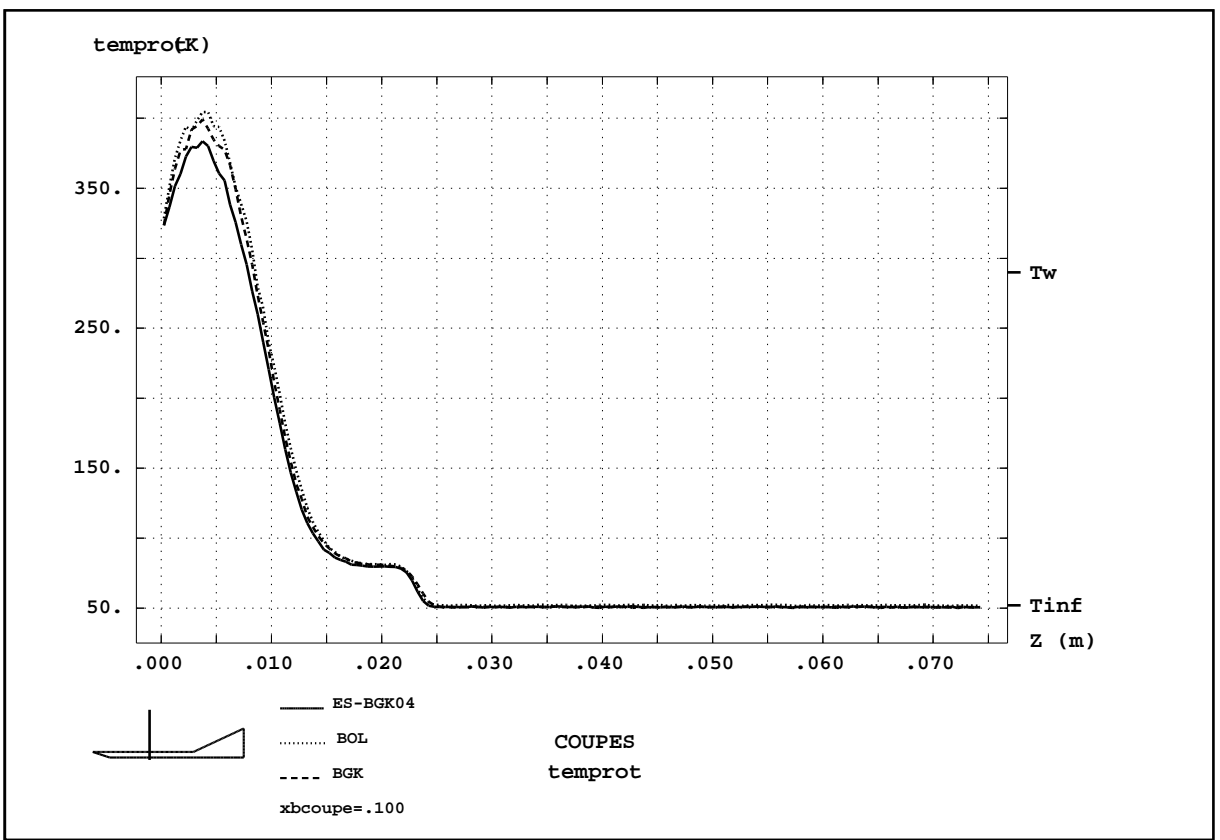

Figure 39: ramp, diatomic, cross section of the rotational temprature at $\mathrm{x}=0.1$

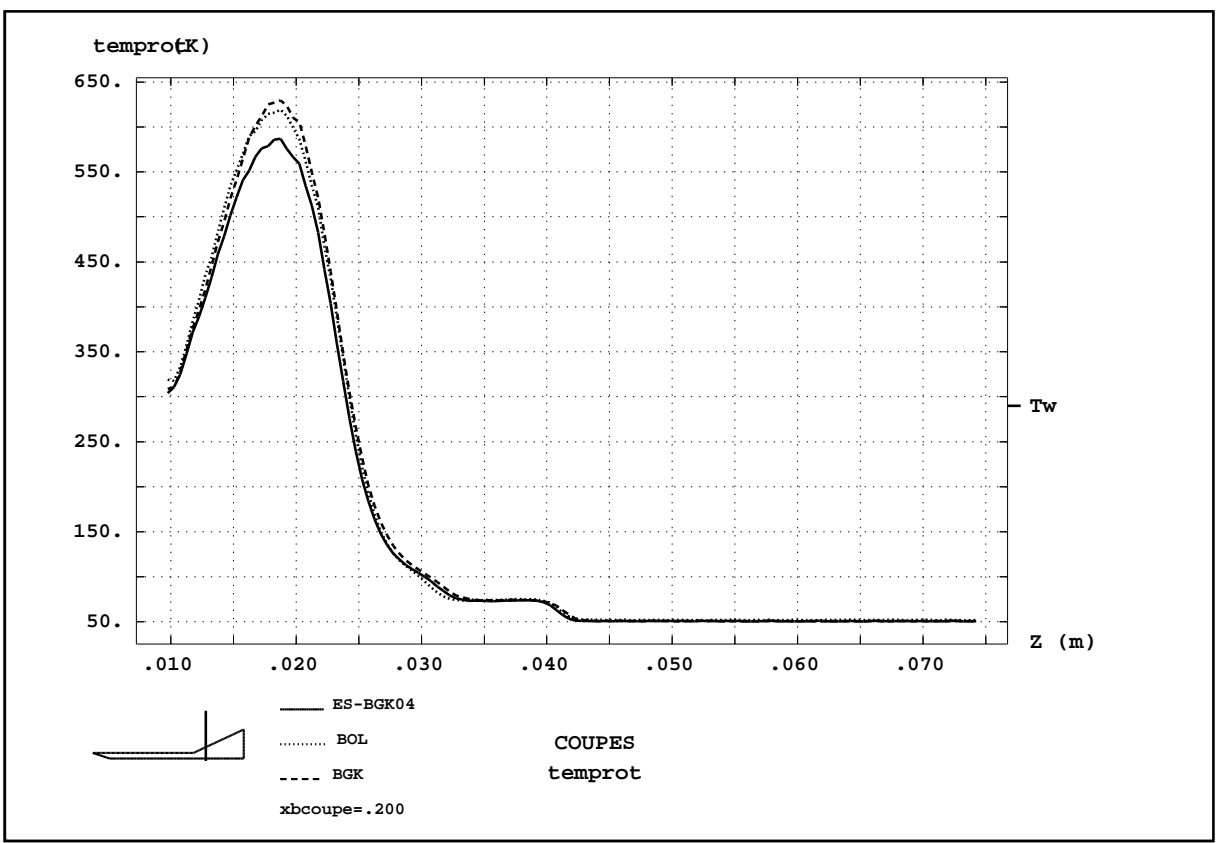

Figure 40: ramp, diatomic, cross section of the rotational temperature at $\mathrm{x}=0.2$ 


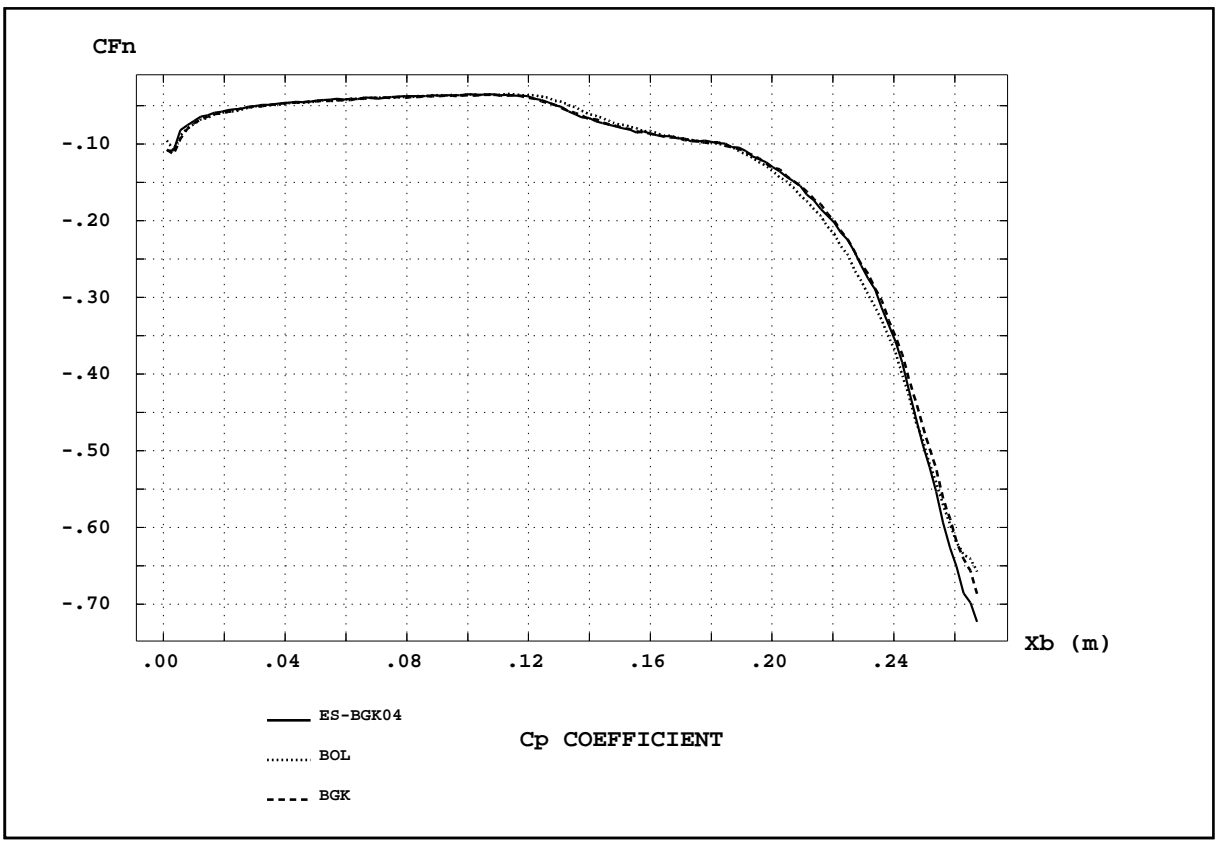

Figure 41: ramp, diatomic, Cp coefficient on the upper wall

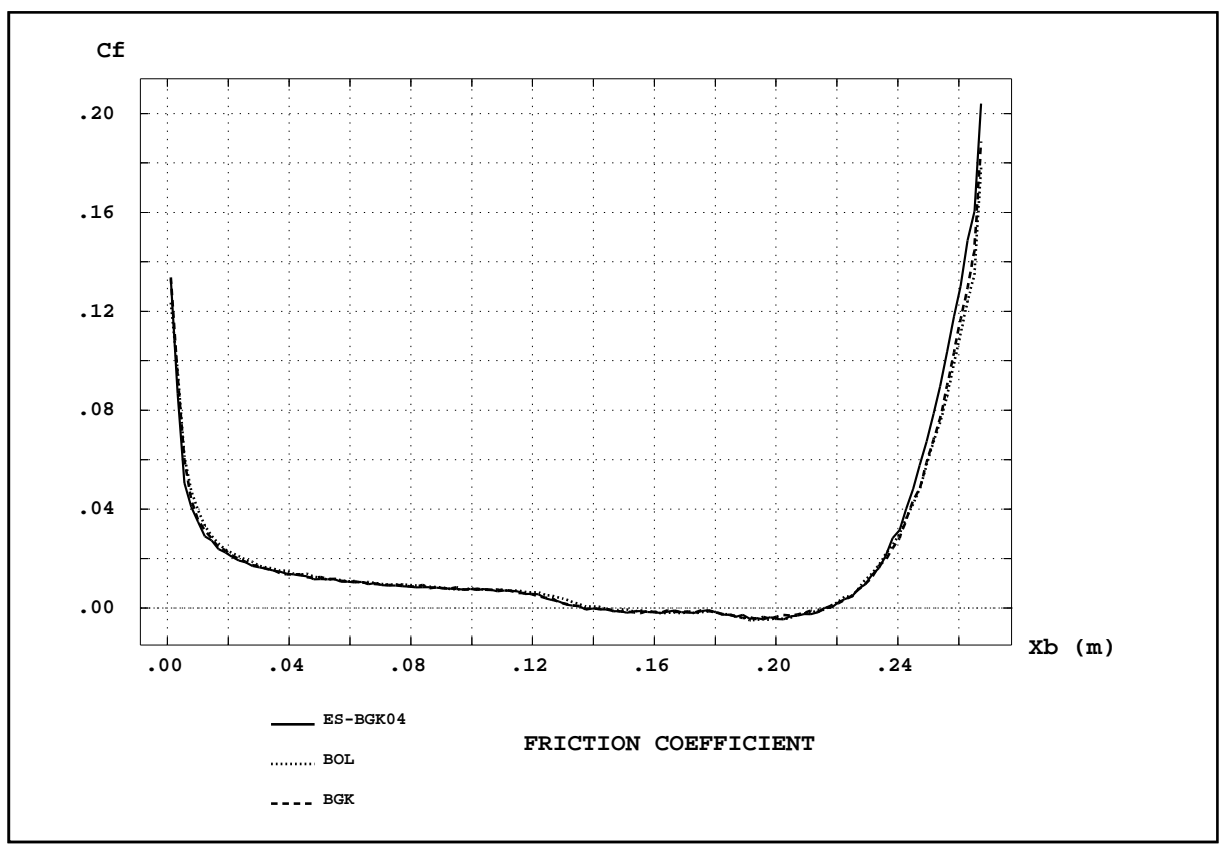

Figure 42: ramp, diatomic, Cf coefficient on the upper wall

$\mathrm{RR} \mathrm{n}^{\circ} 3872$ 


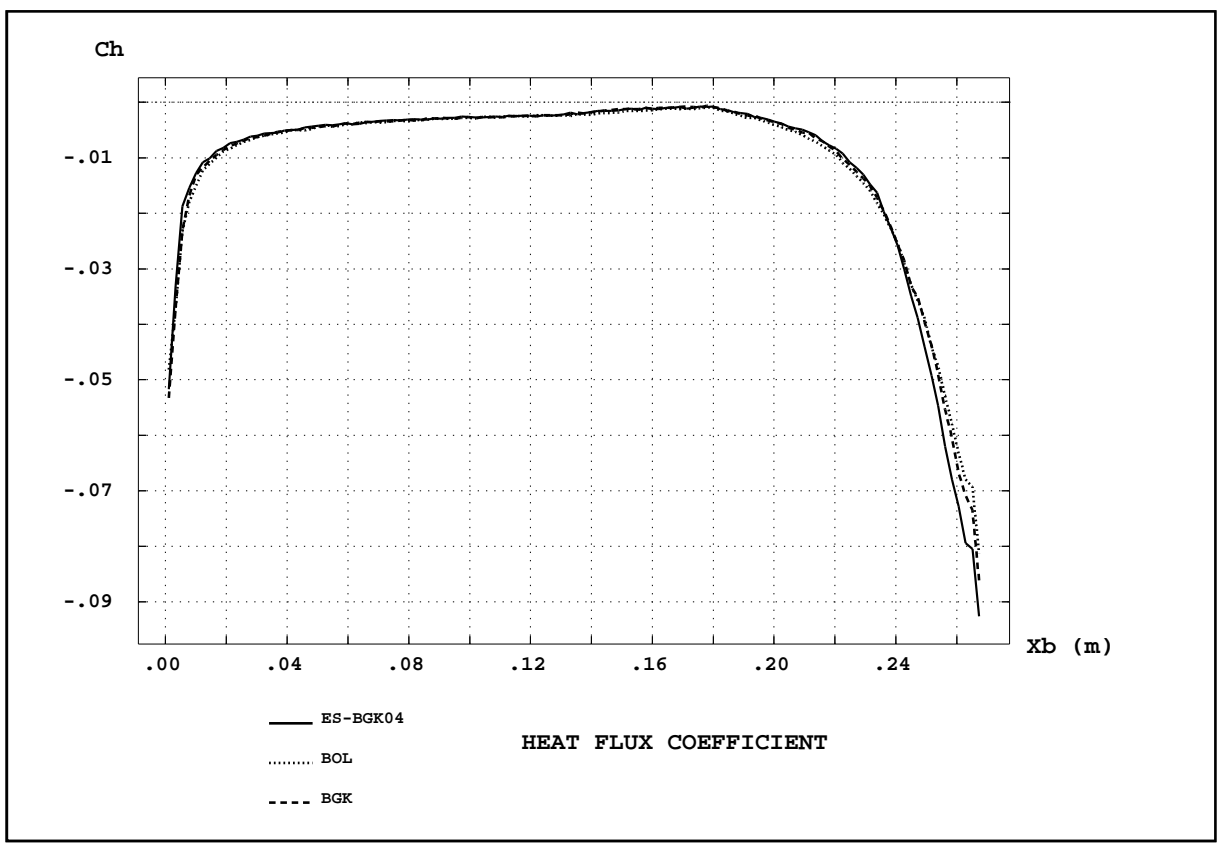

Figure 43: ramp, diatomic, Ch coefficient on the upper wall

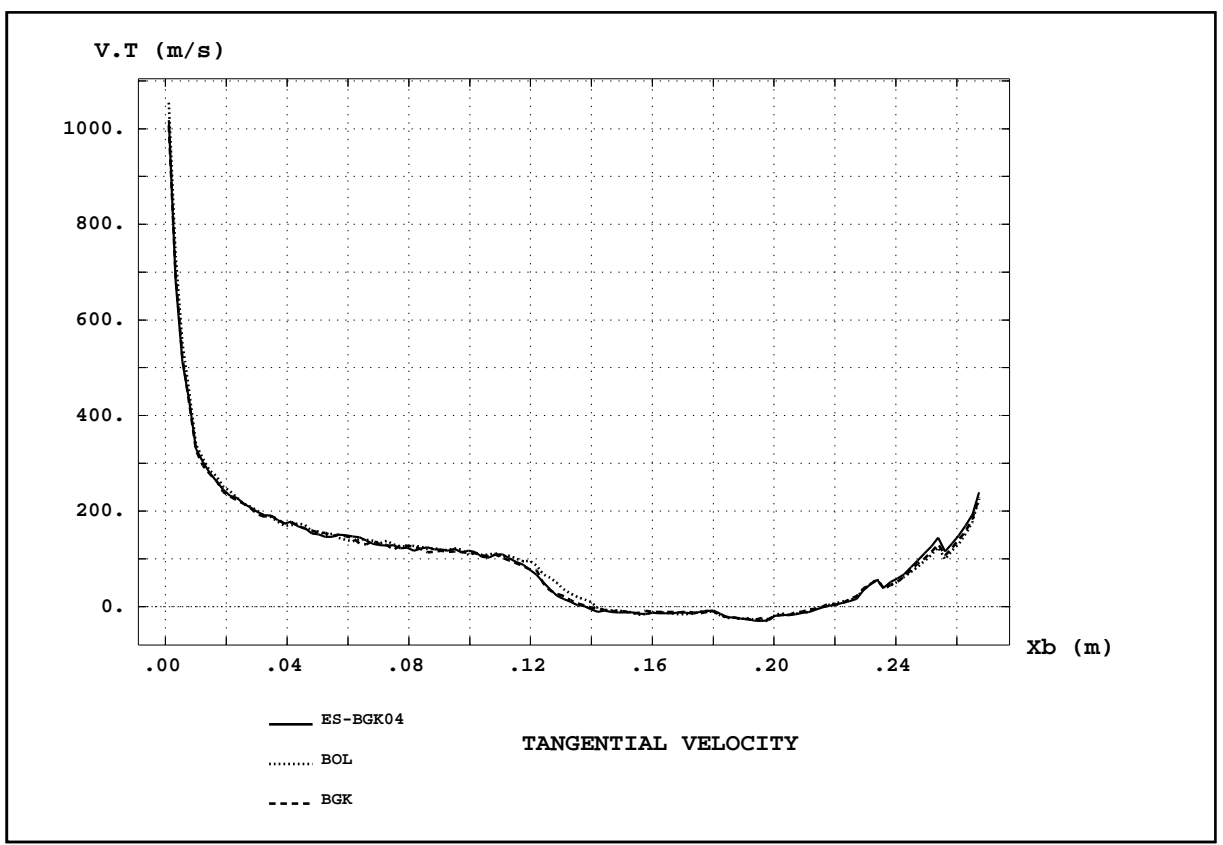

Figure 44: ramp, diatomic, tangential velocity on the upper wall 


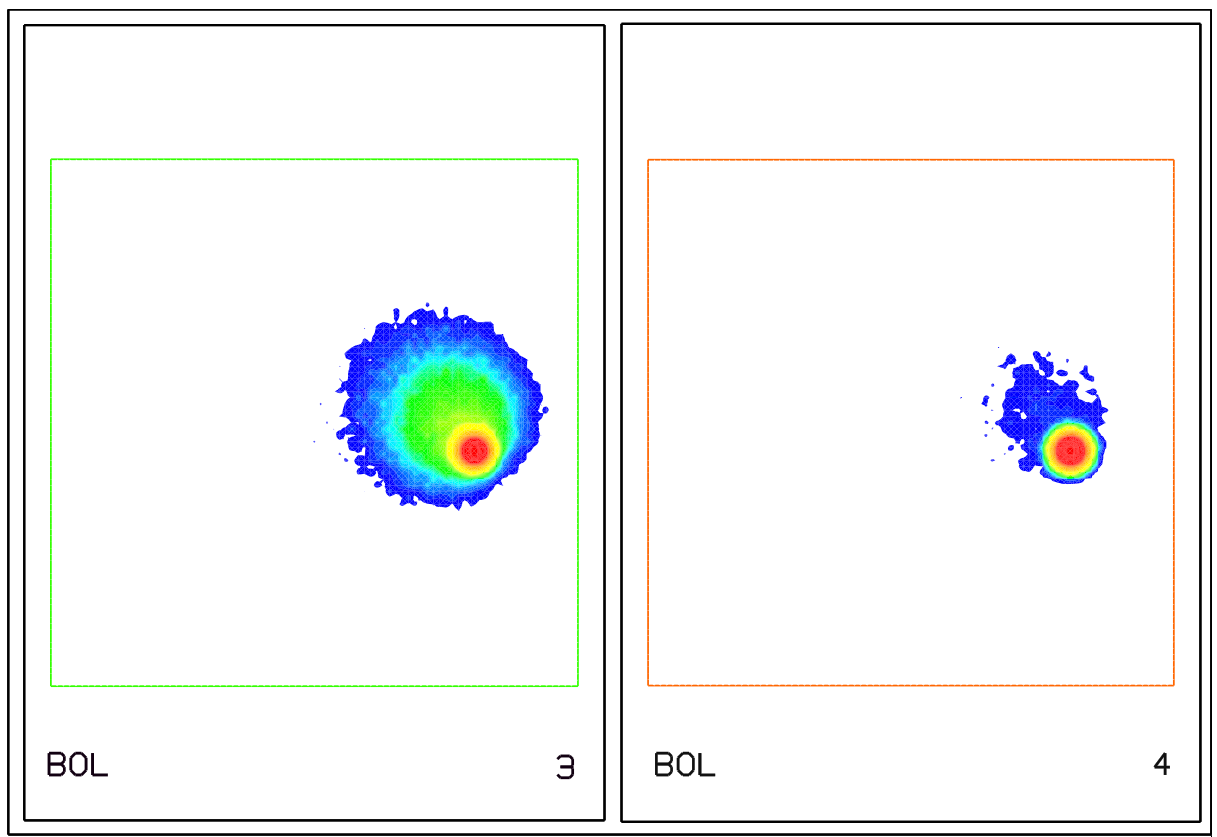

Figure 45: plate, $K n=1.4410^{-2}, 2 \mathrm{~d}$ velocity distribution before the shock(right) and in the shock(left)

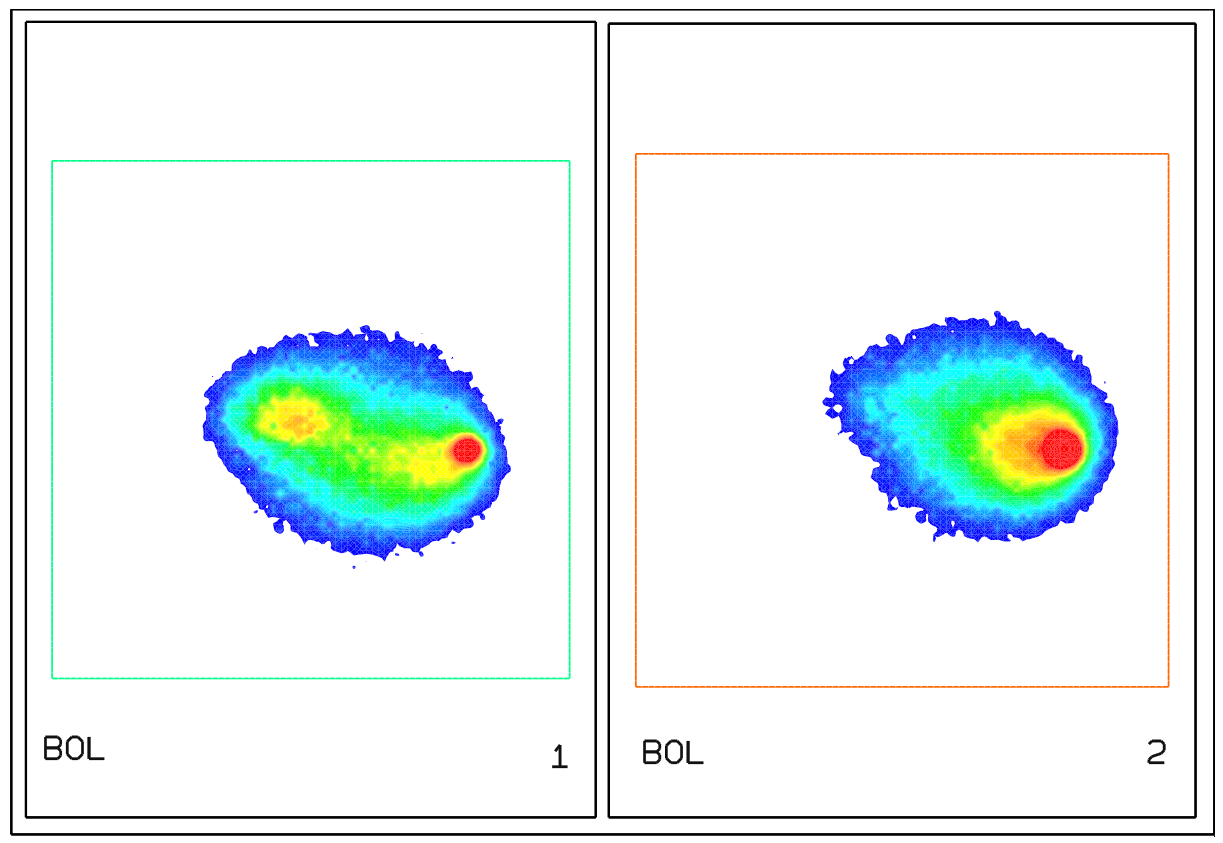

Figure 46: plate, $K n=1.4410^{-2}, 2 \mathrm{~d}$ velocity distribution against the wall(left) and just above(right) $\mathrm{RR} \mathrm{n}^{\circ} 3872$ 


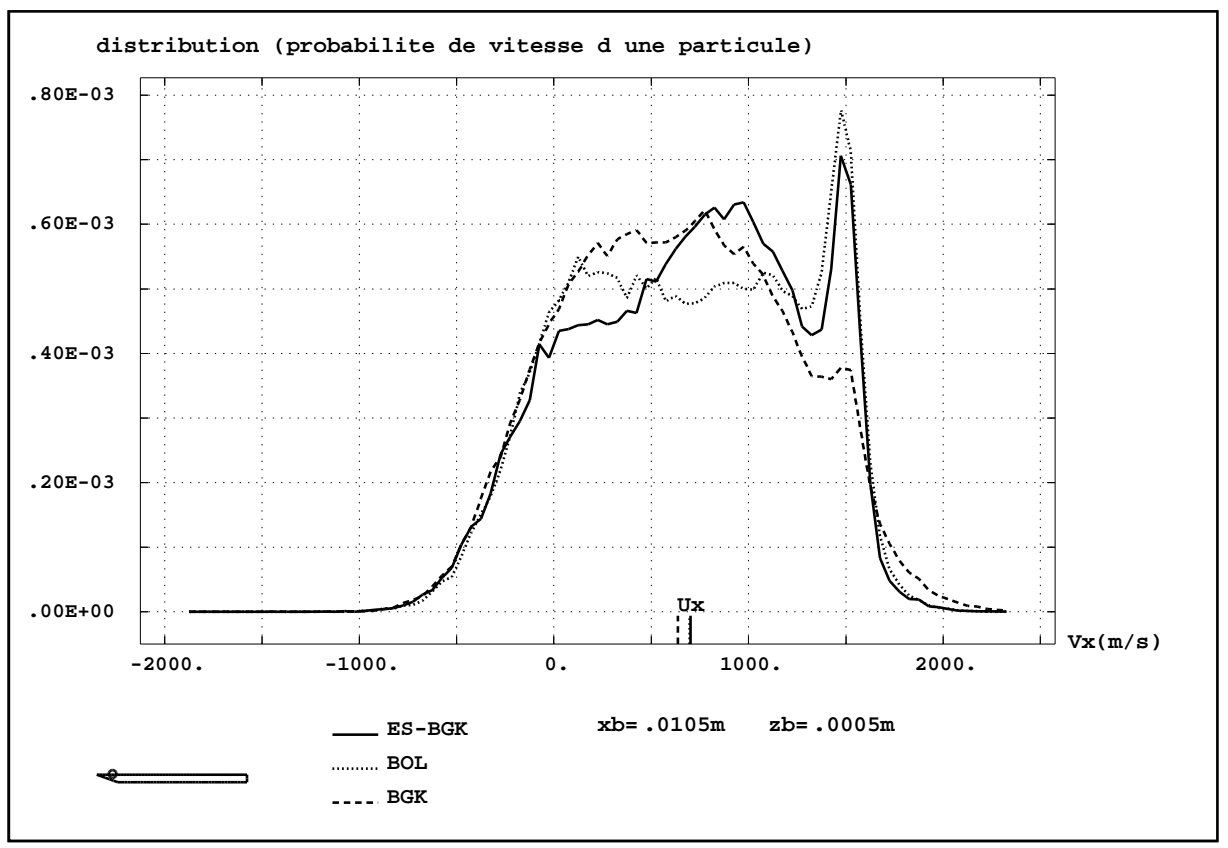

Figure 47: plate, $K n=1.4410^{-2}$, x-velocity distributions at point 1

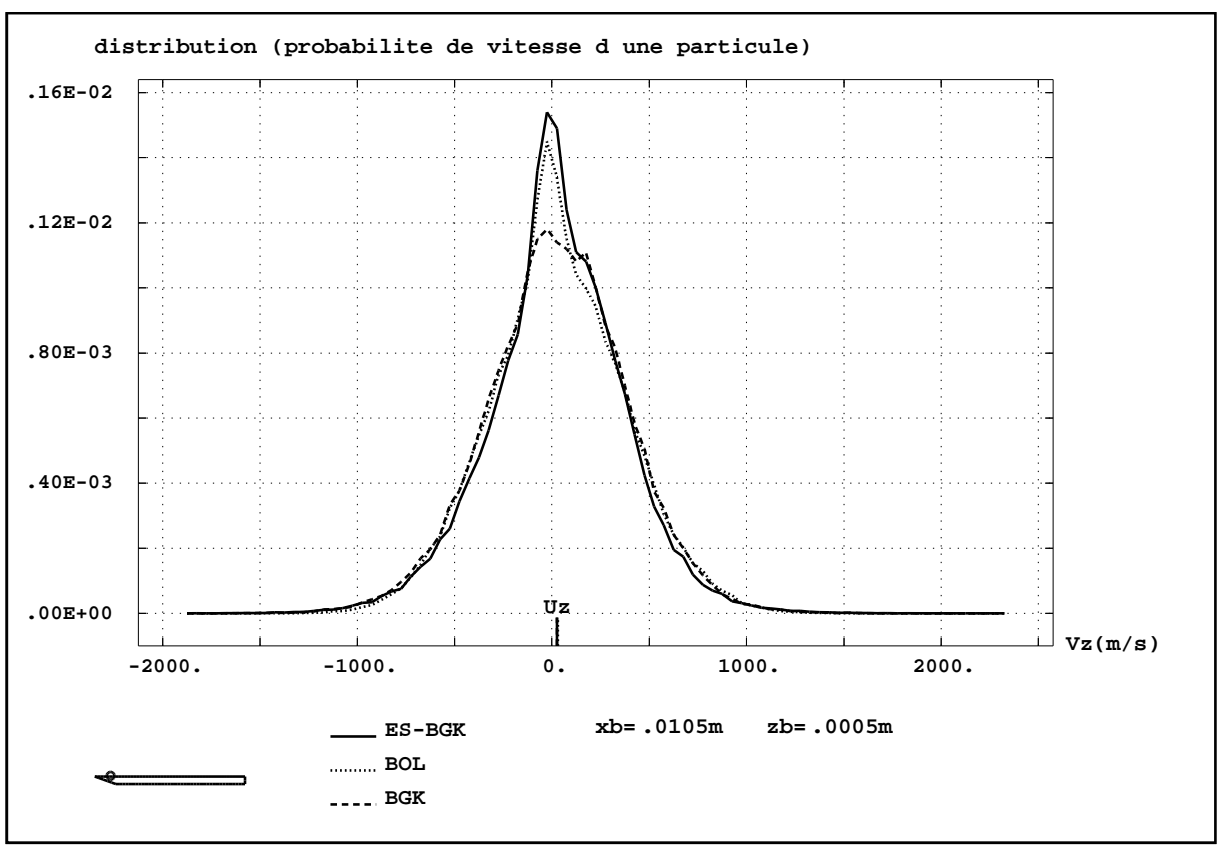

Figure 48: plate, $K n=1.4410^{-2}$, z-velocity distributions at point 1 


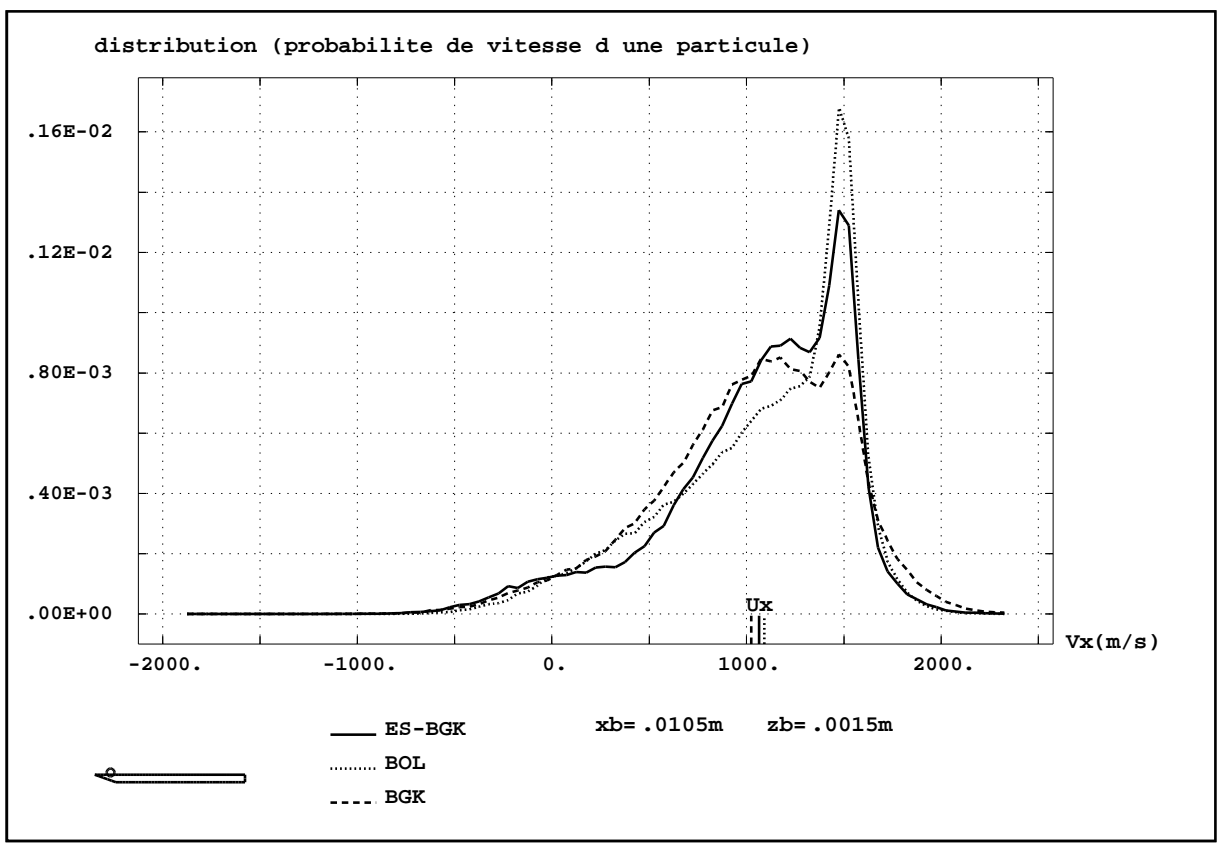

Figure 49: plate, $K n=1.4410^{-2}$, $\mathrm{x}$-velocity distributions at point 2

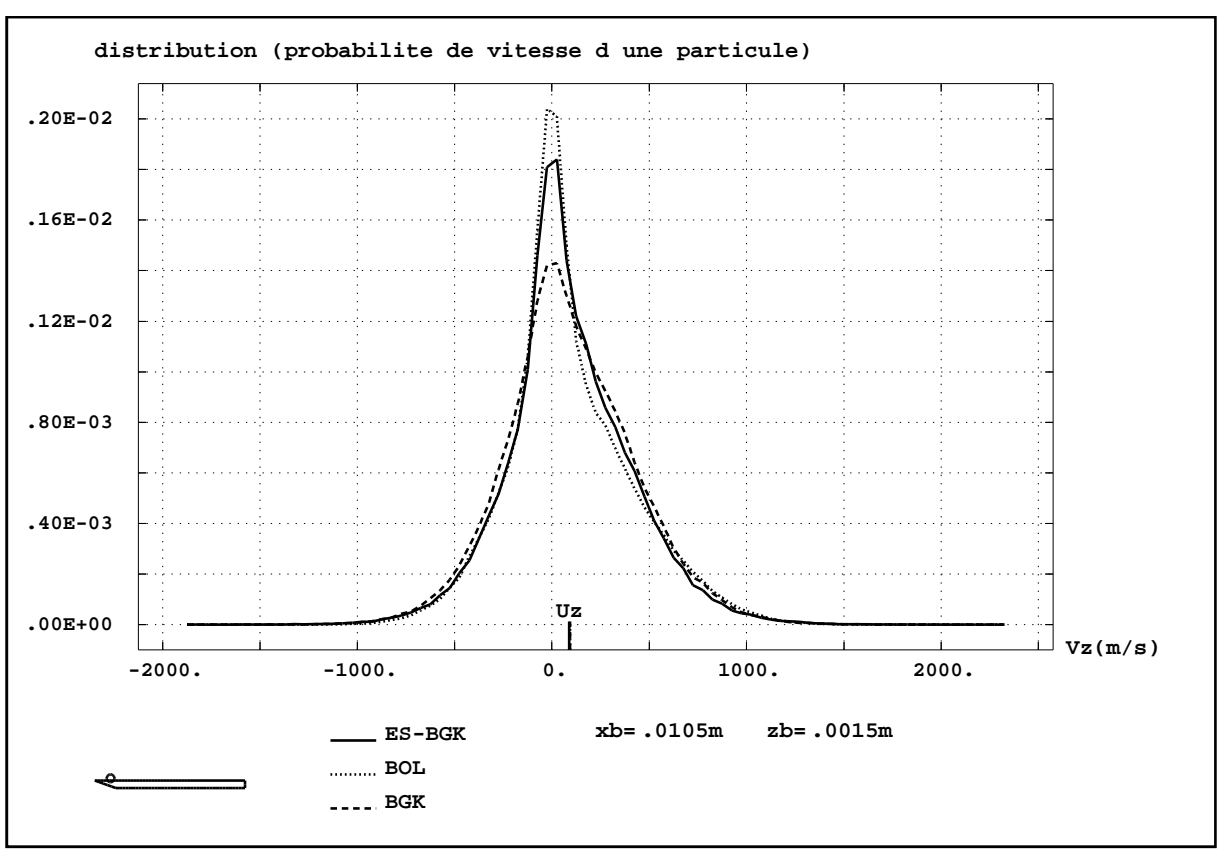

Figure 50: plate, $K n=1.4410^{-2}$, z-velocity distributions at point 2 $\operatorname{RR} n^{\circ} 3872$ 
Unit de recherche INRIA Rocquencourt Domaine de Voluceau - Rocquencourt - BP 105 - 78153 Le Chesnay Cedex (France)

Unit de recherche INRIA Lorraine : LORIA, Technople de Nancy-Brabois - Campus scientiøque 615, rue du Jardin Botanique - BP 101 - 54602 Villers-ls-Nancy Cedex (France)

Unit de recherche INRIA Rennes : IRISA, Campus universitaire de Beaulieu - 35042 Rennes Cedex (France)

Unit de recherche INRIA Rhne-Alpes : 655, avenue de l'Europe - 38330 Montbonnot-St-Martin (France)

Unit de recherche INRIA Sophia Antipolis : 2004, route des Lucioles - BP 93 - 06902 Sophia Antipolis Cedex (France)

diteur

INRIA - Domaine de Voluceau - Rocquencourt, BP 105 - 78153 Le Chesnay Cedex (France)

http://www.inria.fr

ISSN 0249-6399 